\title{
Mineral Asset Valuation Under Economic Uncertainty: A Complex System for Operational Flexibility
}

\author{
Marcell B. B. Silveira
}

Follow this and additional works at: https://researchrepository.wvu.edu/etd

\section{Recommended Citation}

Silveira, Marcell B. B., "Mineral Asset Valuation Under Economic Uncertainty: A Complex System for Operational Flexibility" (2016). Graduate Theses, Dissertations, and Problem Reports. 6645.

https://researchrepository.wvu.edu/etd/6645

This Thesis is protected by copyright and/or related rights. It has been brought to you by the The Research Repository @ WVU with permission from the rights-holder(s). You are free to use this Thesis in any way that is permitted by the copyright and related rights legislation that applies to your use. For other uses you must obtain permission from the rights-holder(s) directly, unless additional rights are indicated by a Creative Commons license in the record and/ or on the work itself. This Thesis has been accepted for inclusion in WVU Graduate Theses, Dissertations, and Problem Reports collection by an authorized administrator of The Research Repository @ WVU. For more information, please contact researchrepository@mail.wvu.edu. 


\title{
Mineral Asset Valuation Under Economic Uncertainty: A Complex System for Operational Flexibility
}

\author{
Marcell B. B. Silveira \\ Thesis submitted to the Statler College of \\ Engineering and Mineral Resources \\ at West Virginia University \\ in partial fulfillment of the requirements for the degree of \\ Master of Science in Mining Engineering
}

Aaron Noble, Ph.D., Chair

John Herbst, Ph.D.

Brijes Mishra, Ph.D.

Department of Mining Engineering

Morgantown, West Virginia

2016

Keywords: Mineral Asset Valuation, Operational Flexibility, Complex Systems, Price

Forecasting, Monte Carlo Simulation, Interior Point Method, Genetic Algorithm

Copyright 2016 Marcell B. B. Silveira 


\begin{abstract}
Mineral Asset Valuation Under Economic Uncertainty: A Complex System for Operational Flexibility
\end{abstract}

Marcell B. B. Silveira

The global mineral industry faces constant challenges that are incited and intensified by market uncertainty. Demand constrictions, resource scarcity, and market volatility, all generate market risk that is compounded by the high capital and long payback periods inherent to mining projects. Quantitative risk assessments provide a methodology to leverage uncertain economic scenarios and accurately assess profitability; however, current mine valuation techniques and engineering economic approaches tend to scrutinize the uncertainty of technical factors, such as ore grade and metallurgical recovery, to a much greater degree than market factors, like price-demand restrictions. Nevertheless, the optimal operating conditions for mining, mineral processing and refining must reflect the true dynamics of uncertain commodity prices, and typical operational responses, such as modifications to mine production and material stockpiling.

This thesis presents a new mineral asset valuation methodology based on economic uncertainty in the commodity market and operational flexibility for mining operations. This novel valuation approach resulted in the generation of a complex system that consists of three primary components. First, a price forecasting component was used to generate future commodity price scenarios with two different stochastic differential equation models (Geometric Brownian Motion and Mean-Reverting-drift). Second, a dynamic methodology of discounted cash flow (DCF) was developed, allowing operational flexibility for mining, processing, stockpiling, and selling material. Third, two distinct optimization techniques (Interior-point method and genetic algorithms) were applied for identification of optimal operating parameters in a mining operation, with a particular focus on using buffer stockpiles to ameliorate the impacts of volatile price fluctuations. The dynamic model was applied in a case study assessing the valuation of a greenfield $\mathrm{Ni}-\mathrm{Co}-\mathrm{Sc}$ mine project. The hypothetical deposit was subjected to different levels of commodity price trends, price volatility, discount rates and maximum stockpiling capacity. Overall, the dynamic valuation model obtained NPV results ranging from $2 \%$ to $11 \%$ higher than standard static DCF techniques. Operational flexibility and ore inventory management proved to be crucial for profit increase on the project. 


\section{Contents}

List of Figures $\quad$ ix

List of Tables $\quad$ xi

Dedication $\quad$ xii

Acknowledgements xiii

1 Introduction $\quad 1$

1.1 Background ........................... 1

1.2 Objectives and Approach . . . . . . . . . . . . . 2

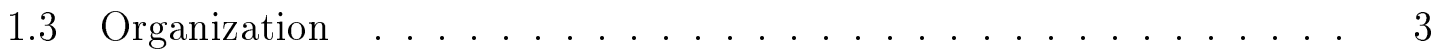

2 Literature Review 5

2.1 Price Forecasting .................... 5

2.1.1 Commodity Market Overview ............ 5

2.1 .2 Price Volatility ................... 9

2.1.3 Stochastic Differential Equations Models .......... 13

2.1.4 Monte Carlo Simulation for Price Forecasting . . . . . . . . 15 
2.2 Computer Modeling for Mine Valuation . . . . . . . . . . . . 16

2.2.1 Mine Valuation Methods ............... 16

2.2.2 Decision-making under uncertainty . . . . . . . . . 18

2.2.3 Material stockpiling . . . . . . . . . . . . . 19

2.2.4 Stockpile Models . . . . . . . . . . . . 20

2.2.5 Mathematical and Computer modeling . . . . . . . . . 21

2.2.6 Optimization and Algorithm Structure .......... 24

2.3 Optimization Methods . . . . . . . . . . . . 25

2.3.1 Multi-variable non-linear solving . . . . . . . . . 25

2.3.2 Genetic Algorithm ................ 26

2.3.3 Interior point method $(\mathrm{IPM}) \ldots \ldots . \ldots 28$

2.3.4 Integrating simulation and optimization . . . . . . . 28

2.4 Summary and Conclusions . . . . . . . . . . . . . . . 29

3 Price Forecasting $\quad 31$

3.1 Stochastic Differential Equation Modeling . . . . . . . . . . . 32

3.1.1 Geometric Brownian Motion ............ 33

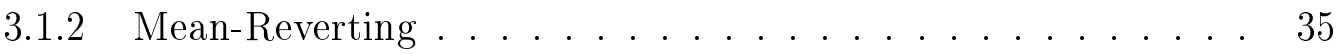

3.2 Simulation of Price Forecasts . . . . . . . . . . . 36

3.2.1 Number of simulations required . . . . . . . . . . 38

3.3 Methodology .......................... 41

3.3.1 Beta index determination ............... 42 
3.3.2 Price sorting, validation and plotting .......... 43

3.3.3 Experiment Design .................. 44

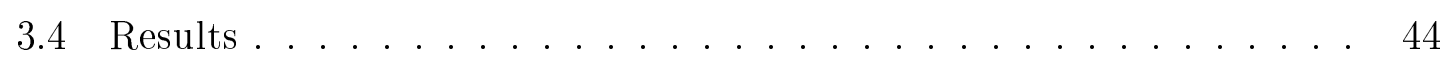

3.5 Summary and Conclusions . . . . . . . . . . . . 48

4 Computer Modeling for Mine Valuation $\quad 49$

4.1 Discounted Cash Flow Model . . . . . . . . . . . . . . 49

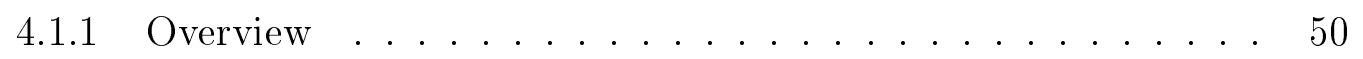

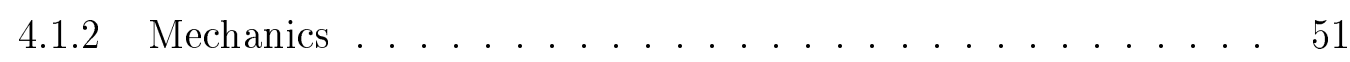

4.1.3 Discount Rate.................... 52

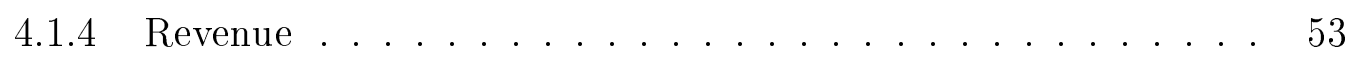

4.2 Real Options Valuation Model . . . . . . . . . . . . 53

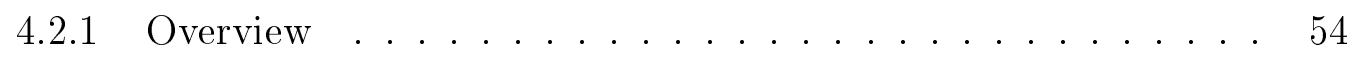

4.3 Dynamic Option Valuation Model . . . . . . . . . . 54

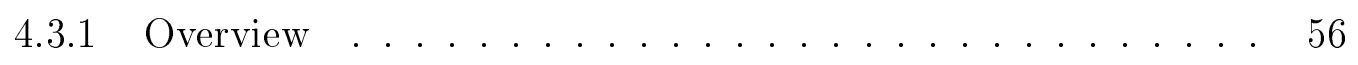

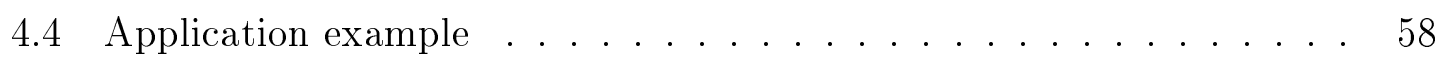

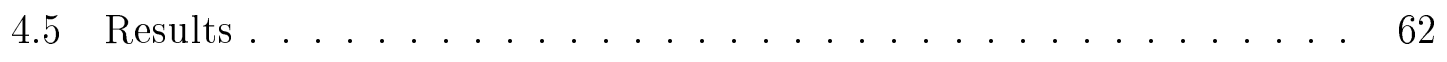

4.6 Summary and Conclusions . . . . . . . . . . . . . 64

5 Optimization Methods $\quad 65$

5.1 Genetic Algorithm ..................... 65

5.2 Interior Point Method . . . . . . . . . . . . 68 
5.2.1 Evaluation of solvers for DOV . . . . . . . . . 70

5.2.2 Determining the required number of function evaluations . . . 71

5.3 DOV Problem Formulation . . . . . . . . . . . . . 72

5.3.1 Dynamic modeling procedure ............ 72

5.3.2 Generating a complex system ............. 75

5.4 Summary and Conclusions . . . . . . . . . . . . . 76

6 Results and Discussion $\quad 78$

6.1 Base study - Pilot scale valuation . . . . . . . . . . . 79

6.2 Case Study - Greenvale Deposit . . . . . . . . . . . . 85

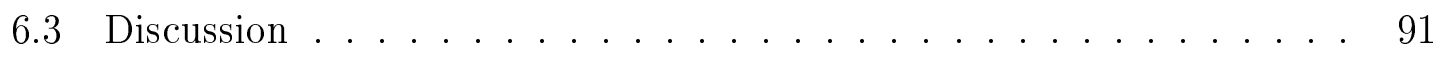

6.3.1 Analysis of Variance . . . . . . . . . . . . 92

7 Conclusion and Recommendations $\quad 94$ 


\section{List of Figures}

2.1 Nickel Price . . . . . . . . . . . . . . . . . . . . 6

2.2 Nickel Supply and Demand versus time . . . . . . . . . . . 8

2.3 Commodity price change along time $\ldots \ldots \ldots \ldots \ldots$

2.4 Complex System . . . . . . . . . . . . . . . . 23

2.5 Genetic Algorithm . . . . . . . . . . . . . . . 27

2.6 Interior Point Method . . . . . . . . . . . . . . . . . 29

3.1 Geometric Brownian Motion model generation . . . . . . . . . . 35

3.2 Linear Regression for Hull-White/Vasicek model determination . . . . 37

3.3 Hull-White/Vasicek model generation . . . . . . . . . . . . . 37

3.4 Simulation example . . . . . . . . . . . . . . . 38

3.5 Price forecasting overview $\ldots \ldots \ldots \ldots \ldots \ldots$

3.6 Nickel price forecasts . . . . . . . . . . . . . . . 46

3.7 Cobalt price forecasts $\ldots \ldots \ldots \ldots \ldots \ldots \ldots \ldots$

4.1 Discounted Cash Flow Model _. . . . . . . . . . . . . 50

4.2 Present value of money for two discount rates $\ldots \ldots \ldots \ldots \ldots$

4.3 Real Option Valuation Model . . . . . . . . . . . . . 55 
4.4 Dynamic option valuation - Feasible operating region . . . . . . 57

4.5 Buffer structures on the dynamic option valuation method . . . . . 58

4.6 Standard vs Dynamic mill throughput example . . . . . . . . . 59

4.7 Commodity prices used on mine valuation . . . . . . . . . 61

4.8 Mine Valuation Methods Comparison . . . . . . . . . . . 63

5.1 Fmincon approximation to the genetic algorithm . . . . . . . . 70

5.2 Required number of function evaluations to reach Fmincon minimum 72

5.3 Interface between mine and mill in the dynamic model . . . . . . 75

5.4 Complex System for Mine Valuation . . . . . . . . . . . 76

6.1 Mine throughput plotting - Base study . . . . . . . . . . 81

6.2 Mill throughput plotting - Base study . . . . . . . . . . 81

6.3 Buffer pile inventory plotting - Base study . . . . . . . . . . 82

6.4 Nickel sales plotting - Base study . . . . . . . . . . . . 83

6.5 Cobalt sales plotting - Base study . . . . . . . . . . . . 84

6.6 Scandium sales plotting - Base study . . . . . . . . . . . 84

6.7 Revenue x Net Present Value Ratio - Base study . . . . . . . . . 85

6.8 Mine throughput plotting - Case study . . . . . . . . . . 87

6.9 Mill throughput plotting - Case study . . . . . . . . . . 88

6.10 Buffer pile inventory plotting - Case study . . . . . . . . . 88

6.11 Nickel sales plotting - Case study . . . . . . . . . . . . 89 
6.12 Cobalt sales plotting - Case study . . . . . . . . . . . . . 90

6.13 Scandium sales plotting - Case study . . . . . . . . . . . . 90

6.14 Revenue x Net Present Value Ratio - Case study . . . . . . . . . . . 91 


\section{List of Tables}

2.1 Beta value interpretation . . . . . . . . . . . . 12

2.2 Mine Valuation Methods . . . . . . . . . . . . . . . 17

2.3 Summary of risk buildup discount rate . . . . . . . . . . . 18

3.1 Nickel price $\log$ differentiation . . . . . . . . . . . . . 35

3.2 Number of simulations required . . . . . . . . . . . . 40

3.3 SDE model modifying parameters . . . . . . . . . . . . 41

3.4 Beta values for Nickel and Cobalt . . . . . . . . . . . . . 43

3.5 Experiment Design ......................... 44

4.1 Greenvale resource table ................. 60

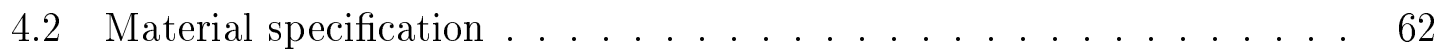

4.3 Capital and Operating Costs . . . . . . . . . . . 62

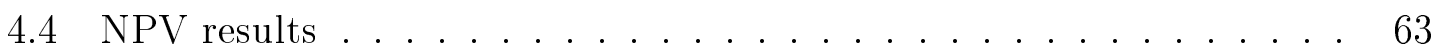

6.1 Base study - Result data . . . . . . . . . . . . 80

6.2 Case study - Result data . . . . . . . . . . . . 86

6.3 Analysis of Variance table - Base study . . . . . . . . . . . . 93 
6.4 Analysis of Variance table - Case study . . . . . . . . . . . . . . 93 


\section{Dedication}

I dedicate my work to my family and many friends who helped me in this long journey. Kara Savage, Hassan Amini, Brunno Almeida, Wesley Edge, Chris Vass, Grant Speer, Aanand Nandula, Neel Gupta, Xue Zhai and Jerica Yingling, I dedicate my work to you and all the many others who allowed me to be successful.

I also dedicate this work to the person who motivated me the most and never stopped believing in me: Aaron Noble. Thank you. 


\section{Acknowledgements}

I would like to thank the committee members who were extremely helpful with their expertise and precious time. A special thanks to Dr. Aaron Noble, my friend, Research Advisor and committee chairman. Thank you for the patience and motivation you gave me to pursue my objectives.

Thank you Dr. John Herbst, for sharing your extensive knowledge in a charismatic way.

Thank you Dr. Brijes Mishra, for giving the example of how successful a young professional can be in such short career time.

Acknowledgements to the Minerals Engineering Technology Development Lab, a group of individuals with high achievement and motivation levels.

Acknowledgements to the Coordination for the Improvement of Higher Education Personnel (CAPES), the Foundation within the Ministry of Education in Brazil whose central purpose is to coordinate efforts to improve the quality of Brazil's faculty and staff in higher education through grant programs. 


\section{Chapter 1}

\section{Introduction}

\section{$1.1 \quad$ Background}

The mineral industry has faced difficult challenges in the recent years. Mining companies are pressured by investors and society to be more productive and profitable under volatile economic scenarios. Mining and its related activities (prospecting, exploration, metallurgy) are very dependent on the commodity prices in the market. Mineral commodities, like other goods, are subjected to the market volatility, changes in supply and demand, and most importantly, the overall economic context of a given time period. Therefore, the mineral industry may be subjected to economic recessions, demand decrease and resource scarcity.

The mineral market is as an attractive long-term investment. Mining projects are subjected to careful mineral asset valuation studies, and only the ones which present profitability in the long term are selected for future development. Therefore, valuation is one of the most important stages of economic analysis. On the other hand, commodity price volaltility may cause decrease in demand, undervaluing the potential of future mining projects. Base metals, for instance, may lose their natural value in times of recession. There is a cyclical nature in the overall mining industry, noticed since the beginning of international trade in the 18th century [Jacks et al., 2009].

Quantifying risk in such volatile market is a challenge that requires specific methodologies. Besides the usual risk of producing a commodity and selling it for a profitable price on the market, it is necessary to be aware of the stock market and its practices. A company can only obtain funding for the development of a mining project if it is 
proven that such operation will be profitable not only on short term, but also on the long term, and will remain profitable for as much time as the investor requires. The value of money over time is also a matter of concern, considering that mineral asset valuation is done with discount rates that exceed $10 \%$ a year [Haque et al., 2014].

Accurate feasibility studies are a recurring theme in mine valuation. The future value of mining project must be calculated carefully to attract investors and obtain funding. The company that intends to move forward with a mining project must perform price forecasting, to identify the economicity of revenue generation based on future market prices. Decision-making is one of the biggest difficulties considering commodity price volatility, and, because of that, operational flexibility must be considered for project valuation.

The current methodologies of mineral asset valuation are primarily based on static discounted cash flows over time [Darling, 2013]. Real Option Valuation (ROV) is considered one of the few methodologies able to measure the value of a mining deposit with the possibility of expanding or shrinking production on a given economic context [Zhang et al., 2015]. However, this methodology is limited to comparing possible operational scenarios and identifying the most profitable outcome on a finite number of possibilities. It is not robust and usually does not account for price volatility or stockpile inventory on a mineral operation.

\subsection{Objectives and Approach}

The main goal of this research is to develop a novel mineral asset valuation methodology, based on economic uncertainty and operational flexibility. The economic analysis can be manifested by subjecting the valuation model to different scenarios of commodity price trend and volatility, generated through simulation. Flexibility, on the other hand, corresponds to subjecting the design parameters of the operation to dynamic valuation, targetting higher economic profit.

In summary, the objectives of this study are to:

1. Investigate and quantify the benefits of operational flexibility under economic uncertainty on mineral asset valuation 
2. Quantify the impact and economic consequences of stockpiling material for future processing and sales while performing mineral asset valuation under a dynamic methodology

The development of the mineral asset valuation model will consist of the generation of a complex system comprising price forecasting, dynamic modeling and optimization methods for mine valuation. Price forecasting will be based on generating future commodity price paths based on two different stochastic models (Geometric Brownian Motion and Mean-Reverting Drift), in order to simulate different scenarios of trend and volatility for commodities.

A dynamic methodology of discounted cash flow (DCF) will be created, allowing operational flexibility of mining, processing, refining, stockpiling and selling material on a mining operation. The combination of price forecasting and dynamic modeling will then provide basis for optimization using two distinct techniques (Interior-point method and genetic algorithm) for the identification of optimal operating parameters under different economic scenarios, targetting increase in NPV. Variable inventory configuration for material stockpiling will be evaluated under this procedure. It corresponds to a new approach in mine valuation, not described previously in other techniques.

Finally, the complex system intends to evaluate the efficacy of different optimization approaches in mine valuation. The objective of the study will be to identify the economic benefits of valuating a mineral deposit using a robust model, which takes in consideration the market behavior for commodity price trend and volatility. The economic gain in using this methodology will be targeted as a mean of evaluating the benefits of such method.

\subsection{Organization}

This thesis is divided into seven chapters.

Chapter 1 is the Introduction, describing the background objectives of the study.

Chapter 2 presents a Literature Review on Commodity Price Forecasting, Computer Modeling for Mine Valuation and Optimization Methods for Mine Valuation. 
Chapter 3 presents a study performed in Commodity PriceForecasting and the development of an algorithm to forecast commodity prices using two different Stochastic Differential Equations Systems.

Chapter 4 presents a study on Computer Modeling for Mine Valuation, focusing on the development of a dynamic mine valuation model used to calculate NPV for mining projects.

Chapter 5 presents a study on optimization methods for Mine Valuation, focusing on Genetic Algorithm and Interior-point methods for problem optimization.

Chapter 6 presents the final results of the study, combining the previous chapters into an overall study on Mine Valuation under price and stockppile configuration uncertainty. It presents a case study on the mineral asset valuation of the SCONI mining project.

Chapter 7 presents the conclusions of the study, as well as recommendations for future work. 


\section{Chapter 2}

\section{Literature Review}

\section{$2.1 \quad$ Price Forecasting}

In this section, a summary of current practices of global mineral trade will be presented. Aspects such as determination of commodities prices, market volatility, types of commodity sales and current innovations in commodity finance will be presented. In addition, a brief analysis of price forecasting methodologies will be presented, as well as current research advances associated to the forecasting practices.

\subsubsection{Commodity Market Overview}

Mineral commodities are products openly sold for subsequent use in industry, which will then transform the material into products with higher degree of alteration. Davis [Davis, 2010] defines that mineral ores generate the first saleable product of mining as mineral concentrates. Mineral ores correspond to a concentration of mineralization located either proximate to the surface or underground. The USGS Mineral Commodity Summary [USGS, 2015] describes a total of 119 commodities whose production, market context, pricing and supply/demand relationship is evaluated in the USA.

Given the low relative price for mineral ores compared to final products, the international market for materials with no alteration is practically non-existent [Hummels and Skiba, 2004] . However, the commodity market for mineral concentrates and final products is very well established worldwide [USGS, 2015], considering the global demand for commodities such as coal, base metals, oil and gemstones. Figure 2.1 


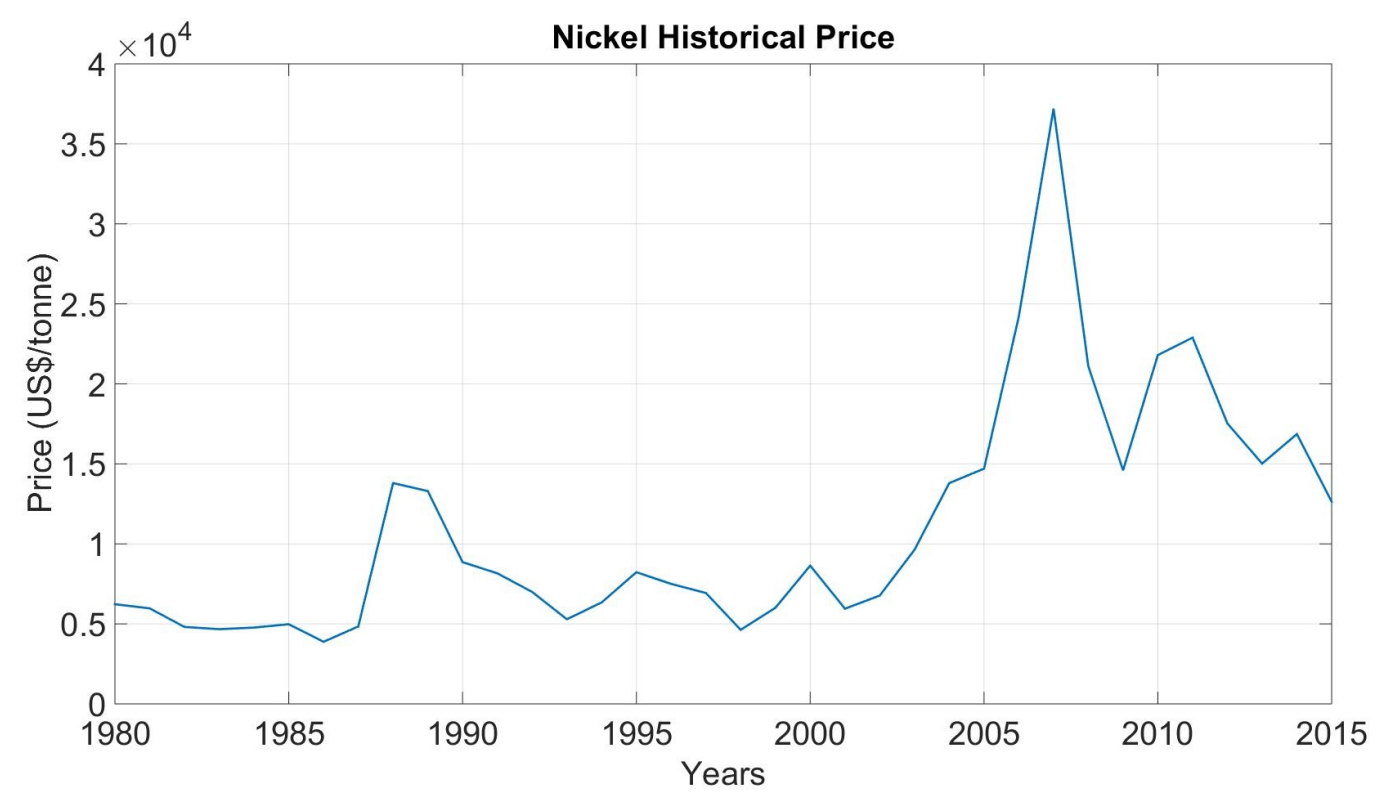

Figure 2.1: Nickel Price

shows the changing prices of nickel on the international market along time.

Mineral commodities are usually produced by mining companies and sold in the exchange market, for a stipulated price. This value is dictated by supply and demand, the type of commodity, and the degree of alteration applied to it. On a background paper to the 2010 World Trade Report, Davis [Davis, 2010] presents a study on the economics of international trade in mineral resources. The researcher evaluates the commodity market and points out that, according to the Standard International Trade Classification, these materials are beneficiated and sold under the "ores and concentrates" category. The differentiation between different types of commodities corresponds to the aggregated value that they contain.

Davis specifies, using a study by Radetzki [Radetzki, 2008], that prices per unit of bulk-scale commodities such as iron ore and steam coal correspond to a fraction of high-aggregated value goods such as coffee and wool. Therefore, they do not contain enough intrinsic value to allow international transportation at economic rates. Industrial minerals (such as crushed stone) have even lower prices, and the only alternative for these commodities to be profitable is through sales to close locations, due to their low value per weight. 
As specified by Radetzki [Radetzki, 2008] previously, there are certain types of commodities that have a preference to be negotiated internationally, as opposed to domestically (with the distinct case of industrial minerals and construction aggregates). The prices for these commodities have obtained great importance in the beginning of the 2000s, after the unexpected and rapid increase of their value in the international market [Cuddington and Zellou, 2013].

Several researchers [Krugman, 2008, Kilian, 2009, Hamilton, 2009] have attributed such abrupt changes in the commodities market to the emergence of BRICs (Brazil, Russia, India and China) and other developing nations, whose rapidly-growing industry impelled the demand for base metals, coal and other mineral products [Tang and Xiong, 2010]. In a paper related to the market of commodities, Tand and Xiong present two distinct views regarding the exaggerated volatility of certain mineral commodities. The researches then specify the two probable causes: (1) Changes in prices caused by supply and demand and (2) Market speculation in commodity indices.

The first view is based on the observation that the demand for mineral commodities peaked by the summer of 2008, and then fell after the economic crisis initiated. The second view, however, is based on an analysis performed by two investigators [Masters, 2008, Commission, 2008], attesting that "the total value of various commodity index-related instruments purchased by institutional investors has increased from an estimated $\$ 15$ billion in 2003 to at least $\$ 200$ billion in mid-2008" [Tang and Xiong, $2010]$.

The growth of both importance, volatility and price values of these commodities influenced the initiation of more intense investigations on the international commodity market, focusing on the influence of commodity prices and the subsequent impact in mining and mineral processing operations that are part of the production chain. Stuermer [Stuermer, 2014] provides accurate evidence on the dynamics effect of supply and demand for a period of 150 years, presenting four different commodities: copper, lead, tin and zinc. The researcher then points out that demand is the main factor responsible for price modifications, while mineral supply has a smaller influence on the factor in analysis. Finally, the investigation concludes that price changes (more specifically, price increase) is caused by quick industrialization, and is a phenomenon that repeats through history. Two main observations can be obtained from this study. The first corresponds to the cyclical nature of commodity prices (with respect to inflation and relative power of purchase), while the second is the principle that prices tend to return to their stable value in the long term. 
The cyclical nature of mineral commodity prices is described on a financial study performed by Cuddington and Zellou [Cuddington and Zellou, 2013]. The investigators developed a mineral market forecasting model, considering an attempt to observe the so-called "super cycles" in commodity prices (cycles within a period of 2070 years), proposed to be an explanation regarding behavior of commodity prices in long period of time. The researchers then conclude that using the economic context and reasonable supply and demand parameters, price dynamics can be simulated.

The results generated by the researchers correspond to an asymmetric price cycle with 20 years of expansion and a peak price that is approximately $250 \%$ beyond trajectories. The creation of the super-cycle proves to be worthy of consideration to study the circumstances associated with changing commodity prices in longer terms.

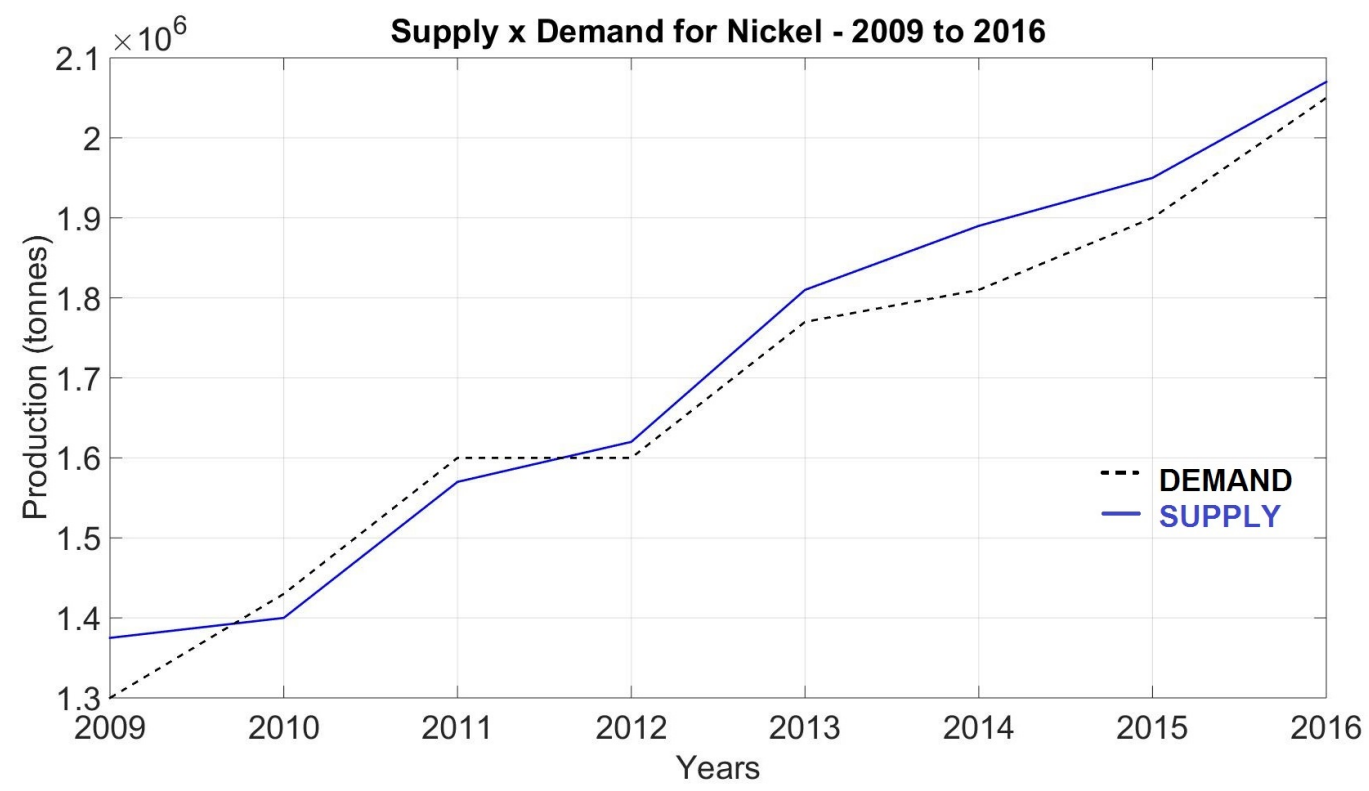

Figure 2.2: Nickel Supply and Demand versus time

Figure 2.2 shows the supply and demand data for nickel since the year 2009, illustrating the gap of supply and demand for nickel due to changing prices. The concerning issue of supply/demand gap for nickel is presented, especially after year 2010. Since more nickel was available on the market at that particular time, prices naturally fell to the ranges that can be noticed nowadays, at around US\$15,000 per tonne [USGS, 2015].

Global demand for nickel seems to be the main driver for the opening and expansion 
of nickel mines, according to Mills [Mills, 2012]. Taking in consideration that nickel is a global commodity, the global players on the international market take demand in consideration while evaluating new projects. The author describes a scenario of global demand growth dominated by China, with yearly increases ranging from 4 to $8 \%$ per year. In North America, the demand is expected to grow at around $7 \%$ per year. Higher commodity prices could be reached in case production delays would happen in current operations.

Given these isues and the magnitude of influence, commodity cycles must be included in mine valuation techniques. The current methods are focused substiantlly in technical parameters and engineering design. A proper mineral asset valuation must take into consideration the impact of price trends on the operating principles of a mining operation, since prices will be the main driver of revenue generation.

\subsubsection{Price Volatility}

Mineral commodity price volatility is a recurring theme both for mining companies and general investors on the commodity market. Gilbertie [Gilbertie, 2012] presents an idea of duality for commodity volatility: They represent a merchandise sold in the international market with a price that is subject to constant change (and consequent inherent volatility), and they also represent a product similar to a stock, in the form of commodity funds. These instruments are traded in stock markets, and contain their own volatility. The definition of a commodity fund is more accurately explained by Smith [Smith, 2008], describing four types of investments:

1. Commodity Funds: Traded in the stock market that hold stocks of mineral assets (such as gold);

2. Commodity Derivative (Options) Funds: Based on the purchase of future stocks;

3. Natural Resources Funds: Based on investment in companies engaged in extracting natural resources (encompassing energy, mining, oil, etc);

4. Combination Funds: Any combination of the funds specified above.

Volatility can be interpreted both as the price volatility of a given commodity in the international market, or also the volatility of a commodity fund. For the 
purpose of this study, the focus will be on the price volatility of mineral commodities. Nevertheless, commodity funds has been addressed by researchers as a topic of great sensitivity. Gilbertie [Gilbertie, 2012] states that commodities are important pieces to increase risk on a stock portfolio, since they are both diversifiers and volatility reducers.

Focusing on the mineral industry, Darling [Darling, 2013] describes that commodity funds can also be considered instruments to evaluate the economicity of a given commodity, while developing mine valuation. Jacks, O’Rourke and Williamson [Jacks et al., 2009] developed a study focusing on commodity price volatility and world market integration since the year 1700. Their research relates the impact of volatility on the destabilization of emerging economies, that are more susceptible to crisis on a volatile market context. This perspective is endorsed by Brown, Crawford and Gibson [Brown et al., 2008].

Figure 2.3 compares the price trend of nickel and cobalt over the course of 32 years. Volatility can be visualized as the measure of price dispersion that a certain commodity may possess in that specified timeframe. In the example, nickel is the commodity that presents a higher volatility compared to cobalt. Even though cobalt has more varying absolute values, nickel presented a higher coefficient of variation.

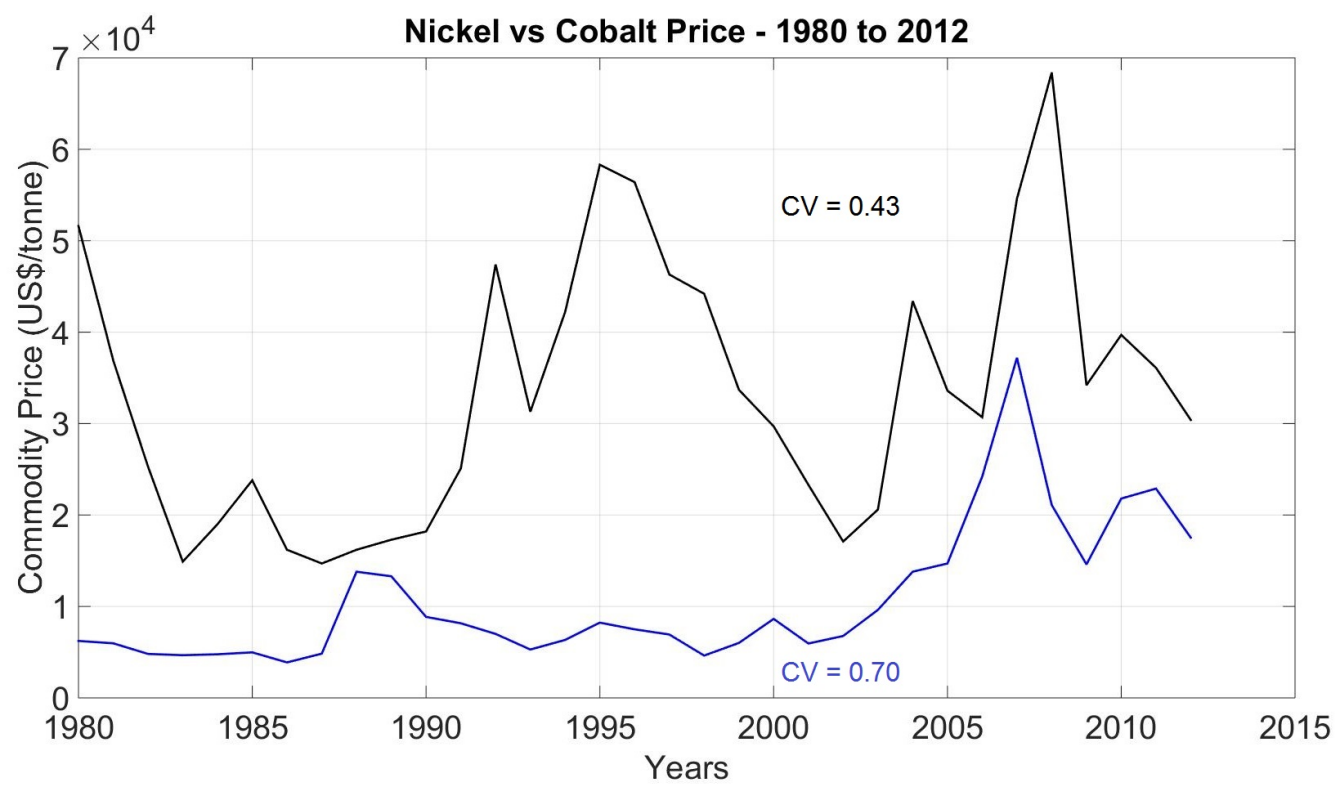

Figure 2.3: Commodity price change along time 
Price specification in a volatile market is examined by Pindyck [Pindyck, 2004] - The researcher investigated the role of volatility in the commodity market, and developed a model for determination of optimal commodity inventory based on prices and market volatility, using data compiled in the petroleum industry. Volatility can be overcome by keeping inventories of oil, and consequently hedging prices in the long run.

A report compiled by the World Bank Group by the end of 2014 [WBG, 2014] shows that "metal prices are expected to decline 5.5 percent, on top of a similar decline last year. A similar decline is forecasted in precious metals as institutional investors are viewing them less attractive as 'safe haven' investment vehicles; reduced demand by China will also contribute to the weakness". This type of language indicates that, even though commodities are an important form of investment, they are currently unattractive because of their declining value on their natural cycle.

The relative volatility of security, portfolio, or equity can be quantified by the $\beta$ parameter [Vardy, 2015]. $\beta$ is a measure of systematic risk for a given commodity, and this analysis can also be applied to minerals, expressed in form of the inherent commodity volatility compared to the overall market in which it is inserted. The volatility of commodities, compared to the overall market, is a relevant parameter for evaluation.

Noble [Noble, 2014] compiled a list of mineral commodity Beta values ranging from 1900 to 2012, identifying the volatility of a certain commodity in the overall market. The formulation for its calculation is the following:

$$
\beta=r \cdot\left(\frac{\sigma_{\text {commodity }}}{\sigma_{\text {overall }}}\right)
$$

where:

- $r=$ coefficient correlation between commodity and overall market

- $\sigma_{\text {commodity }}=$ Commodity standard deviation

- $\sigma_{\text {overall }}=$ Overall market standard deviation

By definition, a $\beta$ of 1 corresponds to the behavior of the overall market. Other valus are explained in Table 2.1 . 
Table 2.1: Beta value interpretation

\begin{tabular}{|l|l|}
\hline Beta factor & Definition \\
\hline$\beta=1$ & Same volatility as the market \\
\hline$\beta>1$ & More volatility in the same direction \\
\hline $0<\beta<1$ & Less volatility in the same direction \\
\hline$\beta=0$ & Uncorrelated to the market \\
\hline$\beta<0$ & Opposite direction to the market \\
\hline
\end{tabular}

Even though the Beta coefficient is a well-recognized aspect on the investment market, some authors consider the range of commodities and their respective trade funds to be limited. Kaplan [Kaplan, 2012] points out that, even though it is relatively easy to invest on a commodity ETPs (Exchange-Traded Products), there are very few choices of selection. A portfolio management must rely on the use and investment in several indexes (The Russell 3000, SP 500, Dow Jones Wilshire 5000, for instance), and commodities lack the versatility that general funds offer, according to the investigator. The perspective is endorsed by Nadig [Nadig, 2014].

Conerly [Connerly, 2014] presents the idea of hedging programs for commodity purchase. The author specifies that companies which purchase commodities should adopt a strategy of hedging prices to smooth price fluctuations while acquiring these products. The concept of hedging prices, but in this case for mineral commodity sale, is presented in the mine valuation methodology presented by Runge [Runge, 1998], [Darling, 2013] and other researchers.

Price trend and volatility have significant impact in mineral asset valuation, especially when future prices must be forecasted through simulation. Trench and Sykes [Trench and Sykes, 2014] present a framework to analyze the impact of commodity price forecasts in future mining operations, proposing opportunities subjected to different market dynamics. Their study focuses on the economics of a mine operations under possible scenarios of uncertainty, describing the importance of contouring instable market prices with pre-determined decisions. The study also presents data regarding the percentage of pages devoted to economic aspects in mine valuation studies performed for four different rare earths projects under the Canadian Mining Code. Approximately $51 \%$ of the content in these reports corresponds to technical details, while the economic and market evaluations are not discussed with the same level of importance, with around $6 \%$ of content only. 
Assessing price risks is consequently not performed with the same extent of detail as the mining and metallurgical factors. The researchers describe that commodity prices fluctuate and are influenced by factors outside the control of a mining company. Factors such as geological and technical problems at mines, labour strikes, mining costs (including fuel prices), infrastructure and environmental issues cause economic impact on the valuation of a mining deposit. Geopolitical factors may also be included in the analysis [Trench and Sykes, 2014].

Expanding on micro-economic level factors, Noble and Luttrell [Noble and Luttrell, 2015] presented a study describing commodity price becoming an important aspect for technical evaluation in coal separation. The researchers present a novel analytical procedure for equipment design, which takes in consideration the overall objective of higher revenue while selecting the cut point in a density separator. This study illustrates the importance of commodity price in engineering design, and subsequent valuation of mineral projects, both under feasibility study or operation.

\subsubsection{Stochastic Differential Equations Models}

The modeling of commodity prices possesses a vast range of possible solutions. As a matter of concern for both the mineral, energy and agriculture industries, it generates discussion in academia and industry regarding the most accurate models to represent prices of commodities [Postali and Picchetti, 2006]. Two methodologies for this application stand out, according to Willingham [Willingham, 2013]. They are both based on Stochastic Differential Equations (SDEs) models: Geometric Brownian Motion (GBM) and Hull-White/Vasicek (HWV).

A stochastic differential equation is a differential equation containing one or more terms as a stochastic process [Oksendal, 2003]. The stochastic aspect will generate models based on random processes, allowing the application of these models in processes that require a wide range of results. Modeling phenomena such as unstable stock prices or physical systems with great fluctuation can be done through these equations. More specifically, commodity future prices and their volatile behavior can be expressed as SDEs, and their values can be generated through simulation.

The HWV model is a gaussian diffusion model with mean-reverting drift [Shreve, 2004]. Values generated from this model possess a random (stochastic) component and also an approximation to the original values presented in the model determi- 
nation. Future paths generated with the model will orbit around a pre-determined mean, usually corresponding to the mean provided in the historical data used for parameter specification. On the context of commodity prices, the historical prices would determine the mean for this model in particular.

Geometric Brownian Motion, on the other hand, corresponds to a stochastic process in which the logarithm of randomly varying quantity follows stationary indepent increments, generated through countinuous-time motion [Glasserman, 2004]. The implications indicate that the values generated through GBM modeling are not affiliated necessarily to the mean of the historical input values. The random aspect is more evident in this model.

Both of the models contain stochastic configuration, however, Geometric Brownian Motion possesses a more randomized characteristic and generally a higher stochastic variance, based on the experimentation made by Willingham [Willingham, 2013]. The Hull-White/Vasicek, as a type of Stochastic Equation Model based on meanreverting drift [Oksendal, 2003], can be used to model commodity prices that are subjected to cycles. The difference between the two methodologies represents the key to evaluate the accuracy of price forecasting with multiple methodologies.

Other researchers have investigated these SDE models and their application on price forecasting. Geman [Geman, ] investigated mean reversion (main principle of HWV) opposed to random walk (main principle of GBM) in oil and natural gas prices, subsequently conducting econometrics studies to conclude if mean reversion is in fact applicable to commodity price behavior. His investigation accepted meanreverting models for modeling future oil and gas prices.

Postali and Pichetti [Postali and Picchetti, 2006] present a study on gas and oil prices as well, focusing exclusively on Geometric Brownian Motion. They conclude that a period between 4 and 8 years (depending on the model set-up) is enough to allow an accurate approximation of a geometric brownian motion pattern. Deng [Deng, 1999] describes the use of stochastic models for price forecasting of nontangible commodities, focusing on electricity prices. The author specifies several different types of formulations for mean-reverting models, varying types of jumps and sparks. Price uncertainty for non-tangible items was also reviewed by the World Bank Group report [WBG, 2014], specifying that energy and food price indices dropped aprroximately $6 \%$ in 2014 .

Yang [Yang, 2012] presents a computer modeling methodology for the develop- 
ment of GBM models using the $\mathrm{R}$ statistical programming language, illustrating that Brownian Motions plays a significant role in building a statistical model. The author points out that complex stochastic models require computer applications for accurate modeling, given their intrinsic complexity. This opinion is endorsed by other investigators [Willingham, 2013].

\subsubsection{Monte Carlo Simulation for Price Forecasting}

Computer simulation is a tool often required while performing sensitivity calculations and simulation on financial aspects of mine valuation [Darling, 2013], given the simplicity of operation while solving complex mathematical equations. Haque, Topal and Lilford [Haque et al., 2014] present a method to construct real option valuation of mining assets through the use of Matlab software. The researchers' final objective is to demonstrate how a GBM model can be used to estimate commodity prices and obtain optimal net present value for a mining project. Apart from the real option valuation, the investigators apply hedging strategies and the use of commodity futures contracts to analyze the proposed scenarios.

Zhang, Nieto and Kleit [Zhang et al., 2015] also perform a similar study on operational flexibility using mean-reverting generated gold prices, in order to determine optimal price threshold for a mine operation. The use of price forecasting proves to be positive considering that, in order to obtain a reliable understanding of the future context for the commodity, it is necessary to generate a forecast.

The topic of simulation has been addressed and investigated by the majority of researchers who deal with options valuations for mineral commodities. The works of Willingham [Willingham, 2013], Nieto and Zhang [Nieto and Zhang, 2013] and others have attested the relevancy of computer simulation on a price uncertainty context.

Monte Carlo simulation corresponds to a methodology of simulation based on using randomly selected input values, allowing the user to investigate distributions of results for processes that rely on uncertain input factors [Mathworks, 2015]. The guidelines for the use of Monte Carlo simulation on the development of properties in advanced exploration or mine valuation scenarios are illustrated [Darling, 2013] and used intensely in industry and academia.

The simulation methodology relies on software applications for calculations and 
statistical analysis. The diversity of software functions, as well as the existence of several packages of interest for creation of SDE models and Monte Carlo simulation is exemplified by Anderson [Anderson, 2004] in his book about MATLAB for Business Economics and Finance Simulation Models. The Financial Optimization Toolbox contained inside MATLAB allows the specification of price forecasting models, especially problems regarding pricing for mine valuation under uncertainty [Willingham, 2013].

Product prices in cut-off grade of a mining operation are evaluated by Nieto and Zhang [Nieto and Zhang, 2013], who exemplify a byproduct mining operation in which both Neodymium (Nd) and Dysprosium (Dy) are considered for NPV calculation. The price variability of the byproduct (Dysprosium) significantly influences the cutoff grade of neodymium. By-products are relevant alternatives to generate revenue and balance capital and operating costs, obtaining more future profit. Commodities such as Rare Earth Elements (REEs) and other rare metals are scarce and highlyvalued on the international market, especially after becoming part of strategic country development guidelines by nations such as United States and China [Humphries, 2013]. Given the high demand and importance of such, there is a supply chain issue that has brought REEs to a higher level of importance.

More specifically on REEs, Nieto and Zhang propose equivalent combined grades for byproducts, elucidating that the price of both commodities ended up impacting the NPV significantly, and therefore the existence of a mineral byproduct can affect the mining strategy completely. Finally, Zhang, Nieto and Kleit [Zhang et al., 2015] exemplify forecasting commodity prices to generate the required results for the Real Options Approach, as opposed to the usual Discounted Cash Flow. Their study, however, was focused exclusively on gold, and does not comprise the possibility of two or more commodities.

\subsection{Computer Modeling for Mine Valuation}

\subsubsection{Mine Valuation Methods}

Mine Valuation corresponds to the study and determination of valuation of a mineral asset. It can be analyzed in many different ways, as presented in the Table 2.2 (after [Darling, 2013]). 
Table 2.2: Mine Valuation Methods

\begin{tabular}{lllll}
\hline & \multicolumn{4}{c}{ Types of Property } \\
\hline Method & Exploration & Feasibility & Development & Operation \\
Market transaction & & $\mathrm{x}$ & $\mathrm{x}$ & $\mathrm{x}$ \\
Market multiples approach & & & $\mathrm{x}$ & $\mathrm{x}$ \\
Replacement cost & $\mathrm{x}$ & $\mathrm{x}$ & $\mathrm{x}$ & $\mathrm{x}$ \\
Real options & & & & $\mathrm{x}$ \\
Monte Carlo Simulation & $\mathrm{x}$ & $\mathrm{x}$ & $\mathrm{x}$ & $\mathrm{x}$ \\
\hline
\end{tabular}

The Real Options methodology was used for the first time in the 1980s, as specified by Shafiee, Topal and Nehring [Shafiee et al., 2009]. It gained popularity because the standard Discounted Cash Flow methodology was insufficient, not being able to account for external factors such as commodity price changes, commodity scarcity, and the observation that deposits, especially the ones containing REEs, can contain more than one commodity.

Cut-off grade has always been considered a relevant factor in the industry. However, the use of Discounted Cash Flow for its determination is potentially inaccurate, according to Caldentey, Epstein and Saure [Caldentey et al., 2004]. Therefore, a more sophisticated approach for those considerations is necessary. The potential of expansion or reduction of production in a mining operation must also be evaluated. Shafiee, Topal and Nehring [Shafiee et al., 2009] conclude that the successful evaluation of a mining project consists in identifying the most economically viable option, which may include expansion, reduction, or even abandonment. A more flexible decision-making methodology can reduce economic risks, according to the researchers.

Supply bottlenecks for the rare earth elements has been addressed by Nassar et al [Nassar et al., 2015], in which the researchers discuss the impact of commodity demand while developing mineral asset valuation for projects with REEs as byproducts. Flexibility must be addressed by selecting the material to be mined and sold, based on the market demand, and not on the overall mining and processing rate. A study in production scheduling with uncertain supply has been addressed by Ramazan and Dimitrakopoulos [Ramazan and Dimitrakopoulos, 2013]. 


\subsubsection{Decision-making under uncertainty}

Uncertainty in the mineral industry has been addressed in several expertise areas, with different approaches and wide application ranges. Montiel and Dimitrakopoulos [Montiel and Dimitrakopoulos, 2015] describe risk-based solutions that outperform deterministic ones, for mining complexes with multiple processing and transportation alternatives.

A special regard on the balance between return and risk is specified by Runge [Runge, 1998]. The author describes the criteria used for the determining the appropriate discount rate to be used at a DCF valuation. He affirms that low discount rates represent more confidence on the economic viability of a mining operation, however, they do not represent the best solution for valuation.

Technical and economic parameters under uncertainty on a mining operation (commodity prices, ore grade, metallurgical recovery, final sales) are also backed up by a high discount rate. Darling [Darling, 2013] presents risk-buildup for discount rate, which corresponds to a process in which the discount rate for a mining project is dismembered in several aspects, one of each corresponding to a risk characsteristic. See Table 2.3.

Table 2.3: Summary of risk buildup discount rate

\begin{tabular}{ll}
\hline Item & Rate, \% \\
\hline Real risk-free rate of return & 2.5 \\
Public company risk premium & 7.0 \\
Small cap premium & 3.0 \\
Industry-specific risk & 2.5 \\
Site-specific risk & 3.0 \\
Total (pre-tax) & 18.0 \\
Total (after-tax) & 12.0 \\
\hline
\end{tabular}

Darling also exemplifies economic principles for decision making, describing that a narrow focus on technical issues is not enough to guarantee economic success, under even with the a high-grade mine deposit. The researcher points out several methodologies to develop strategies in mining, including cost reduction, understanding the time value of money, and establishing a discounted- cash-flow analysis. 
The investigator uses a conservative discount rate of $15 \%$ for his calculations, which is a value synchronized with the principles specified previously by other researchers evidencing risk. Given all of these specifications it is a relevant idea to use a conservative discount rate for the project.

\subsubsection{Material stockpiling}

Material stockpiling is one of the issues addressed by mining professionals seeking higher productivity, quality and cost reduction. It is normally used both for ore, waste and product in the mining industry. Stockpiling for ore, as specified by Warnelid [Warnelid, 2011], is largely used in industry to ensure material homogenization, and subsequent grade variability reduction. In addition, the existence of a stockpile will ensure that the subsequent stages of mineral processing will contain material to be fed when required. Basically, the raw stockpile acts as a buffer for the milling process.

Several studies have been conducted on the optimization of materials handling and stockpiling configuration, in order to allow ease of handling and subsequent target achievement for feed grade. Darling [Darling, 2013] specifies a typical formulation for cutoff and stockpile optimization, in which the determination of material allocation is performed in a way to achieve maximum NPV, considering that there will be higher revenue generated by certifying that material tonnage and grade will be optimal.

The optimization procedure has been described in a study conducted by Asad [Asad, 2005], in which the researcher develops an optimization algorithm to maximize the NPV of a given mine deposit, considering uncertainty of mineral grade, and the shifting of cut-off grade based on maximum mining, milling and sales capabilities. The investigation relies on procedures determined by Dagdelen [Dagdelen, 1992], and contains three assumptions largely accepted while developing mine valuation on a given mine deposit:

- Constant price for the commodities

- Immediate product sales

- Bottleneck between mine and mill (and smelter, in the example) 
The target of the study (cut-off grade optimization) is largely mentioned, however, the uncertainty principle of stockpiling tonnage is not addressed by the researcher. In fact, another researcher [Rendu, 2014] develops a meticulous study on cut-off grade estimation, presenting capacity constraints and opportunity costs. They correspond to the opportunity to decrease the mining or milling cut-off grade in order to generate higher NPV values during optimization. Marginal grades of material are then shifted from the category of waste to ore, depending on the conditions and parameters specified on the study.

A recurring theme in by-product mine valuation, specially regarding REEs, is supply chain constraints that such commodities possess on the international market. Nassal, Graeder and Harper [Nassar et al., 2015] investigate the production and marketing of by-product metals that are used in specialized functions and have very limited supply. The researchers introduced companionality, which corresponds to "the degree to which a metal is obtained largely or entirely as a by-product of one or more host metals from geologic ores. The dependence of companion metal availability on the production of the host metals introduces a new facet of supply risk to modern technology".

The main point addressed by the investigators is that, given the extremely limited supply of such commodities, their location, and finally the extremely low rates of end-of-life recycling, these commodities in particular may represent an economic challenge. The conclusion obtained by the investigation is that, even though these products are technologically essential for the industry, their extraction and use must be modified, otherwise they may become unusable, due to substitution or new technology development.

\subsubsection{Stockpile Models}

As specified previously, the use of stockpile models in mine valuation and operation is usually assessed to investigate cut-off grade examination. However, the models can also be analyzed for different applications. Three main definitions for the use of stockpiles are accepted in the mining industry [Darling, 2013] :

- Blending stockpile: Built and maintained for the purpose of material homogenization. Material is stored for an unspecified amount of time, and keep 
constantly blending the pile with increments from run-of-mine or other materials, in order to achieve grade, volume, or other relevant targets for subsequent processes (usually mineral processing). They are used for all sorts of material

- Grade stockpile: Maintained for the purpose of postponing low-grade material. This methodology is applied usually for the purpose of NPV increase in later years. Increments are deposited over the course of many years for posterior milling. The process is normally associated to precious and base metals

- Buffer stockpile: Designed to act as a buffer between two connected processes, such as mine to mill, mill to smelter or smelter to sales. They are smaller compared to the ones specified previously, and are usually located just beside the beginning of a mineral processing circuit. All types of commodities are associated with type of stockpile.

Even though the stockpile types represent a wide range of applications and possibilities on a mining project, there is no specification of stockpiling methodologies or procedures for material whose value can increase along time. The grade stockpile usually holds material that possesses no economic value in the present. However, there is defficiency in investigation on developing stockpiles for optimal revenue after sales, considering the modification of money value along time, and also changing commodity prices. Finally, it is relevant to point out that the management of stockpiles is possible following two different practices [Mohammad, 1997, Mohammad and Khan, 2004]:

- Stockpile is built with increments parallel to the mining operation. Consequently, the material can be sent to the mill either from the mine operation or the stockpile (depending on the ore characteristics)

- Stockpile was built during the operating years, and its material is only used after the mine is exhausted. All material is sent to the processing plant, coming from that single source

\subsubsection{Mathematical and Computer modeling}

The use of computer applications for mine valuation (on a broader range, mine valuation in general) has been widely recognized both in industry and academia. Darling [Darling, 2013] demonstrates the use of computer applications in the Mineral 
Valuation chapter of the SME Mining Engineering Handbook. The author specifies the use of conventional spreadsheet models, manipulated by an user (engineer, geologist or analyst) who usually creates models with best-case, worst-case, and average-case scenarios. The researcher then illustrates that single spreadsheet models are not the most accurate representation of the complex array of possibilities that a mining project requires. His conclusion is that the use of simulation methodologies (such as Monte Carlo) allows analysis of the full range of possible outcomes, given the capabilities of computer software.

Statistics and simulation prove to be a successful method for the development of mine valuation. Haque, Topal and Lilford [Haque et al., 2014] use the MATLAB software and explicit finite difference method (FDM) to develop a numerical study for a mining project under commodity price uncertainty, as well as an operational flexibility methodology. Willingham [Willingham, 2013] develops a mining economics case study for iron ore, using systems of SDEs to forecast iron prices, and using the forecasting functions present on MATLAB. Anderson [Anderson, 2004] presents several different methodologies for mathematical and simulation in project valuation in his book, focusing on MATLAB for economics and finance. The use a high-level technical computing language and environment for algorithm development is praised in this case.

Finally, Willingham [Willingham, 2013] presents a visual representation of mine valuation using MATLAB interchangeably with Microsoft Excel. Easy data importing on both platforms stands out as a major aspect for the software selection, considering that Excel is the most recognized spreadsheet software available for general use. The researcher points out the several capabilities of MATLAB, its intrinsic programming language and the toolboxes available in different packages. In order to calculate NPV distribution, develop modelling and simulation of future prices and interest price paths, as well as develop risk assessments based on distribution of economic outcomes, the following packages are used: Econometrics, Financial, Optimization and Statistics and Machine Learning toolboxes.

Compiling discounted cash flow models, price forecasting through the use of SDE models, and optimization in a parallel structure requires the use of a system in which the components interact with each other. The works developed by Mohammad and Khan [Mohammad and Khan, 2004] and Willingham [Willingham, 2013] present results with a wide spectrum of distributions and scenarios. This plurality of processes and practices allowed the definition of the system as a complex system. [Richards et al., 1998]. 
A complex system comprises a usually large number of iteracting entities, processes, or agents dependent of each other for the generation of results. Simon [Simon, 1995] further enhances the definition of the model by specifying that it corresponds to a system of many components with many interactions among them, and subsequent dependent behavior. The differentiation between complex systems on a mathematical modeling perspective can be understood in Figure 2.4.

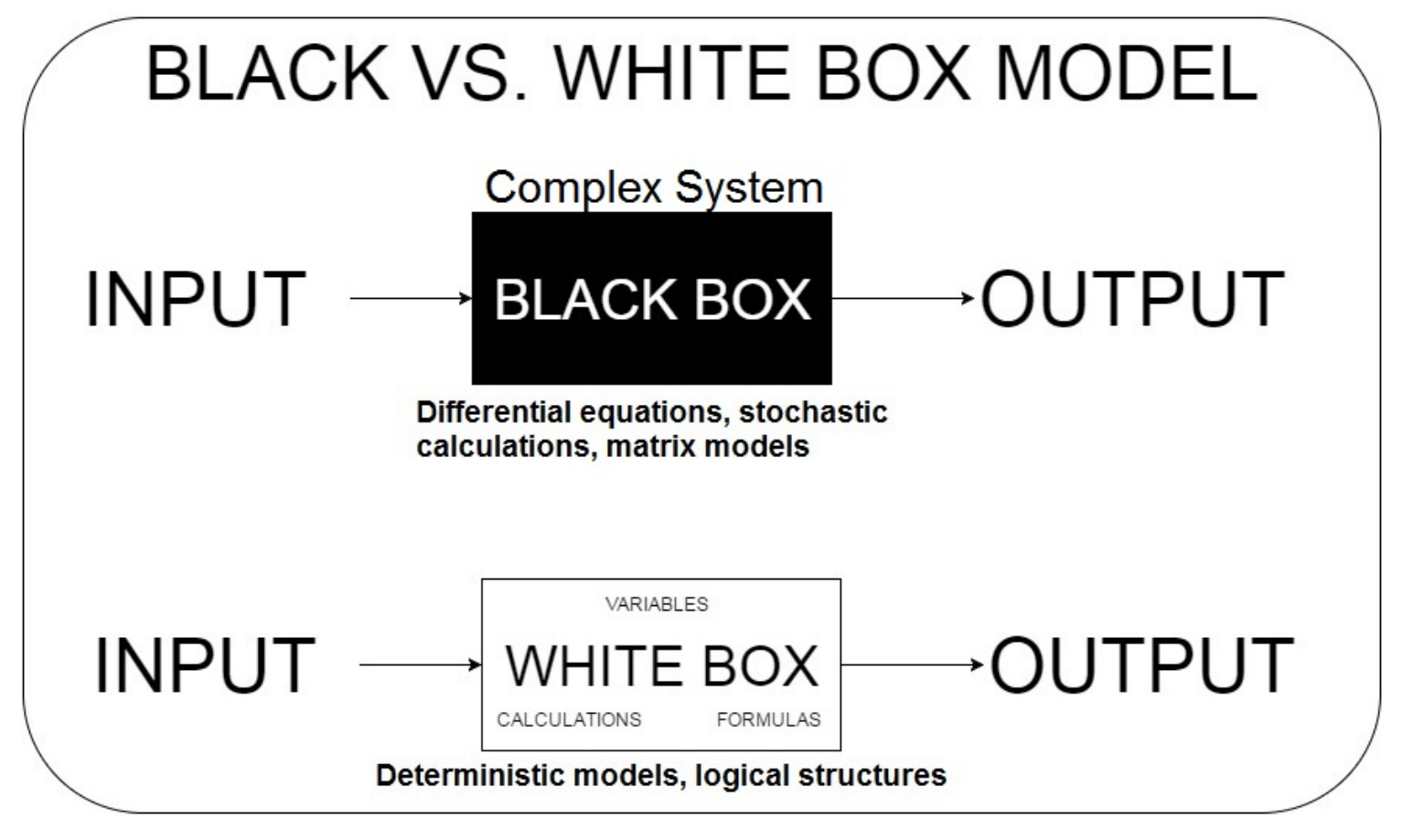

Figure 2.4: Complex System

Veld [Veld, 2014] described complex systems in Financial Economics. The author exemplifies the use of Monte Carlo simulation and robust optimization on the stock market and other financial venues as a means to evaluate these markets and obtain data for investment and decision-making. Treating the financial market as a complex system proves to be an efficient methodology, considering the level of complexity that the globalized financial market possesses.

McDaniel and Driebe [McDaniel and Driebe, 2005] address the problem of operational flexibility on a financial complex systems by using real option valuation in their research. Finally, Hassan [Hassan, 2005] proposed a similar methodology to investigate the value-at-risk of a complex system composed of an aerospace satellite, 
describing the use of flexibility in financial decision making.

\subsubsection{Optimization and Algorithm Structure}

The use of optimization for mine valuation is a recurring theme both in industry and academia. Regarding the construction of algorithm methodologies for mine valuation. Maximizing the NPV for a proposed project is the main objective. For other applications, or standalone cases, the algorithm is set up for other purposes (such as minimizing the operational cost for equipment or maximizing recovery on the processing plant).

Researchers use linear programming and mixed-integer linear programming functions for the development of optimization. Dagdelen [Dagdelen, 1992], Mohammad and Khan [Mohammad and Khan, 2004] present a simple methodology for the calculation of optimal NPVs on a fictitious mining project. The objective function of the optimization algorithm is then presented as maximization of the NPV, subjected to several constraints. The majority of these constraints include non-negativity for mine, mill and sales throughputs, while the corresponding variables of interest are usually associated with the following:

TIME VARIABLES:

1. Total number of project years

2. Total operating years for each process (mining and milling)

3. Time steps in consideration (usually in years)

TECHNICAL VARIABLES:

1. Metallurgical recovery

2. Production Rate (Mine and Mill Throughputs)

3. Commodity Grade

ECONOMIC VARIABLES: 
1. Discount rate

2. Commodity Price

3. Natural Resources Funds: Funds that invest in companies engaged in extracting natural resources (encompassing energy, mining, oil, etc)

4. Costs (Fixed, Variable, Capital, Operating)

The algorithm then calculates the optimal NPV for a given scenario by identifying the most appropriate production rate for processes, the one that will generate higher overall profit and maximize the value of money along time. The decision variables, consequently, will correspond to the throughputs. This standard methodology contains the major assumption that the material produced by the smelter (or processing plant, depending on the case) will be sold in its totality. It does not take in consideration the operational flexibility that a possible delay in sales could generate.

\subsection{Optimization Methods}

\subsubsection{Multi-variable non-linear solving}

Solving optimization problems is a recurring theme in financial modeling, especially the ones that involve some type of non-linear models [Vasant, 2012, Brandimarte, 2006]. The main topic of relevance discussed in this section will be the use of global optimization versus local optimization.In general terms, global optimization promotes search in all the spectrum of possible scenarios, while local optimization starts from a pre-determined location and works in a serial way to find a minimum.

Muller [Mueller, 2004] presents a differentiation between these two methodologies, specifying that the global approach can be serial or parallel in its search structure, while a local approach must be serial. Two solvers will be discussed in this section: genetic algorithm (global optimization) and linear search (local optimization). 


\subsubsection{Genetic Algorithm}

Genetics is a means by which each individual evolves. Evolution can also occur because of learning or random structural or functional alteration, which corresponds to the concept of mutation. The individual adaptation, in this context, corresponds to the idea that the individual is able to survive in a given environment, considering its fitness.

The genetic algorithm is a mathematical algorithm inspired on the natural evolution mechanism and genetic recombination [Holland, 1975]. This provides a search mechanism based on adaptation conditions similar to Darwinism reproduction, survival of the fittest and genetic mutation. Input parameters are subjected to fitness evaluation, and values that better fit the criteria are selected for reproduction.

Subsequently, the values are subjected to crossover and mutation, with a new population being generated. The process continues until the fittest population is discovered. Contextualizing on a mine valuation context, operational parameters (mine and mill throughputs, for instance) are subjected to mutation until the algorithm discovers the values that generate the highest NPV for a given mineral deposit.

The International Seminar on Mineral Processing Technology compendium [Gupta et al., 2005] describes the genetic algorithm as a probabilistic parallel search algorithm based on natural selections. Focusing on the optimization principle of the genetic algorithm, the evolution principle corresponds to reaching values closer to the target (minimization or maximization, depending on the objective). Figure 2.5 corresponds to visual representation of the working process of a genetic algorithm.

In the mineral industry, the genetic algorithm is used for modeling mining and metallurgical operations, given its versatility and adaptability to different contexts. Indian researchers [Gupta et al., 2005] state that "virtually every technical discipline, from science and engineering to finance and economics, frequently encounters problems of optimization".

Considering that the mining industry deals with parameters that are usually estimates and predictions based on models (ore grade, future commodity price, metallurgical recovery equipment efficiency, among others), there is an expertise gap to be fulfilled by the genetic algorithm and its capabilities.

The most usual approach to optimization on the mining industry corresponds to 


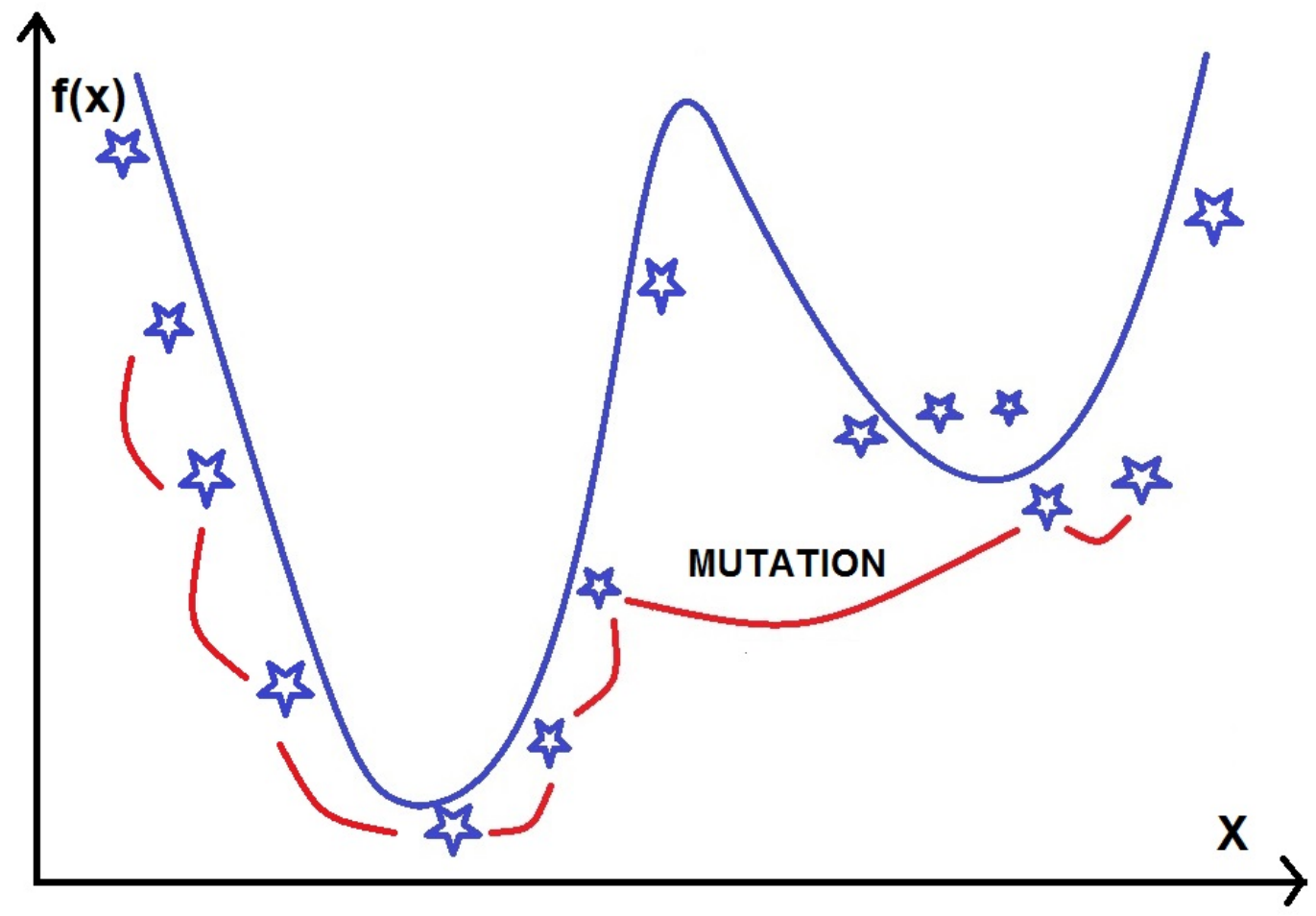

Figure 2.5: Genetic Algorithm

NPV maximization by modification of operation parameters [Gupta et al., 2005, Yun et al., 1990]. However, other correlated activies (extraction, processing, refining and sales) have been addressed in academic research. Investigators [Yun et al., 1990] use the evolutionary system for the optimization of underground mine development. The application proves to be succesful in a chinese gold-copper mine for long-range mine planning.

Crow, Clean and Cresser [Croew et al., 2006] use the technique to promote robust optimization of soil organic and mineral fraction densities, assuming that such problem requires non-linear solving to achieve its purpose. In the context of mineral exploration, Rahman, Dutta and Smith [Rahman et al., 2013] present a novelty application of genetic algorithm for obtaining mineral domains. They evaluated gold assay and geochemical datasets obtained from a gold mine in Western Australia. The experimental results demonstrate that the genetic algorithm was able to identify domains accurately. 
Salim and Cai [Salim and Cai, 1997] developed a methodology for the optimization of railway scheduling with environmental considerations, illustrating that basically every technical principle of a mining operation can be modeled and optimized via evolutionary algorithms. Even though the genetic algorithm is as an interesting methodology for process optimization, and its global optimization aspect is praised by many researchers [Goldberg, 1989, Vasant, 2012], the computational power required by the algorithm is a major topic of concern [Chen, 2002].

\subsubsection{Interior point method (IPM)}

The interior point method is a a non-linear solver capable of identifying the near global optimal solutions for fitness functions, with strong approximation to global convergence [Vasant, 2012]. More specifically on the MATLAB platform, the nonlinear solver is named Fmincon. This algorithm is a method that relies on finding minimum values for continuous and constrained functions, whose main criteria is possessing continuous first derivatives. The interior point method was well described by Powel [Powell, 1983] and explained by Vasant [Vasant, 2012].

Brandimarte [Brandimarte, 2006] states that Fmincon may not be most suitable for certain optimization models, and therefore its results must be compared to a global optimization algorithm. Kienitz and Wetterau [Kienitz and Wetterau, 2013] specify that the Fmincon requires specific lower and upper boundaries for constraints. However, optimization problems with any constraint type can be solved by the commands specified on the solver declaration. Figure 2.6 represents the line search principle of Interior Point Method and Fmincon.

\subsubsection{Integrating simulation and optimization}

The combined use of simulation and optimization methods may seem redundant, since both methods have the same basic purpose, which corresponds to finding optimal values for variables in a model. However, the use of both methodologies combined has been addressed by numerical methods investigators.

Brandimarte [Brandimarte, 2006] exposes the idea that integrating both method-

ologies is worthy of consideration, given that it provides a way to optimize complex 


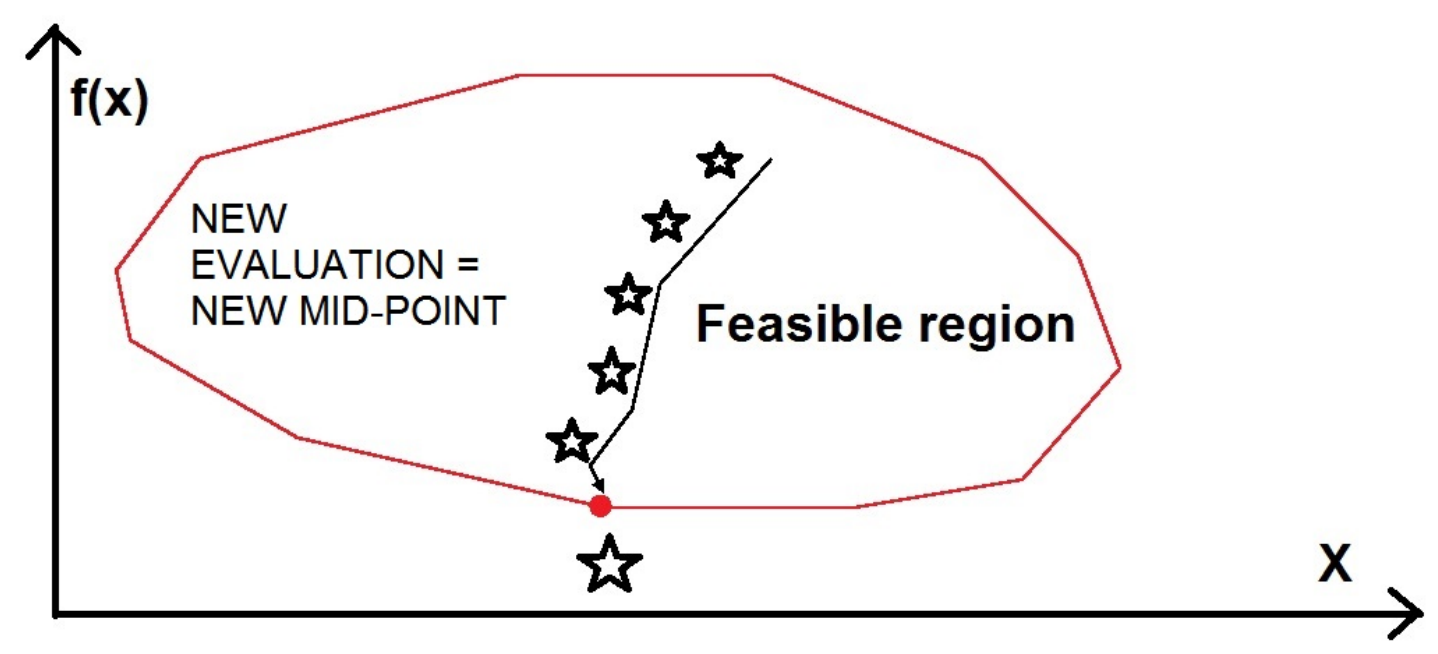

Figure 2.6: Interior Point Method

and stochastic systems, which cannot contain stochastic evaluation in some part of their models. The investigator concludes that non-linear programming methods that do not use derivatives are interesting for simulation optimization, given their time efficiency.

Mine valuation happens to be an interesting topic for optimization, and the delimitation of specific events (referred as "scenarios" and "cases") inside a stochastic complex system instinctively requires the use of a simulation optimization methodology. This perspective is corroborated by Law and McComas [Law and McComas, 2000], who describe a simulation optimization methodology to account for a set of single events contained within an optimization problem.

\subsection{Summary and Conclusions}

Mining companies often require several different types of financial considerations for decision making while evaluating mineral commodities for potential mining, processing and sales. This valuation process requires years of data collection, technical evaluation and financial considerations regarding the value of the desired commodities. 
Commodities are negotiated on a global scale and, as objects of international trade, they are subjected to the inherent volatility present on the market, containing a value subjected to supply and demand needs. Since a potential mine operation can last more than 30 years, it is necessary and relevant to establish predictions of the commodity prices along time. Willingham [Willingham, 2013] specifies that the future economic potential and associated risks of a mineral project are better understood with an accurate risk/return prediction.

The commodity prices will be used by mining companies to establish cash flows over time, and determine the economic valuation of a given mine deposit, considering the amount of material it contains, specifying how much material will be sold on a given time. This valuation methodology is classically defined as Discounted Cash Flow (DCF). In general terms, DCF is a valuation method used to estimate the attractiveness of an investment, considering the Net Present Value (NPV) that the given mine deposit can obtain, given its technical aspects, and the market considerations, while considering the time value of money.

Commodity price will be the ultimate factor to be analyzed while evaluating a mineral project, since the price of a certain commodity will be the necessary parameter required to calculate revenue for a mining project, allowing management to decide whether that project is economic or not. Considering the life of such operation, determining accurate forecasts for price will be extremely resourceful to evaluate the actual value of a mine project. The uncertainty is inherent to the market and must be observed or projected.

The current valuation methodologies are limited because they are either too focused on commodity grade or too simplistic in regard to optimization procedures. In addition, they are majoritarily based in deterministic simulation processes, which are classically less robust than stochastic methodologies. The following shortcomings can be described: (1) Lack of financial studies based on material stockpiling for future processing and sales; (2) Limited use of complex financial studies for project feasibility, based on evaluation of commodity cycles and volatility.

Stockpiling material, allowing a buffer pile to control the inventory, and attempting to generate more profit over time is a topic of great consideration in a cyclical mining industry. The commodity market must be evaluated with the same level of complexity as the technical parameters of engineering equipment and processes. 


\section{Chapter 3}

\section{Price Forecasting}

Economic uncertainty is a relevant theme in mine valuation, particularly when dealing with varying commodity prices. The most important parameter to be input in any mine valuation study is the commodity price, which will influence all other major decisions regarding the economic viability of a mineral deposit. Price forecasting corresponds to the use of techniques to anticipate future prices for a given commodity whose value is dictated by the open market, and the process is largely used in all types of market trade, with special focus in commodities in the context of this research.

Bowman and Husain [Bowman and Husain, 2004] present the importance of forecasting prices for commodities, indicating that commodity price forecasts are essential for planning and project design. Fluctuation in prices may affect global economy, and have significant impact on macroeconomics, considering that countries which produce and export them obtain a significant amount of their export earnings through commodity sales. The researchers' objective was to assess the accuracy of price forecasts for a specific group of commodities, evaluating whether different methodologies were in fact able to predict the price of a mineral commodity on a specific period of time.The researchers also identify three different types of forecast in their analysis:

1. Judgemental forecast, based on quantitative or qualitative external factor analysis (e.g.: supply/demand)

2. Forecasts based on statistical models based exclusively on historical price

3. Forecasts that attempt to rely on all the possible data (including future prices) 
Unlike the study specified above, the purpose of this work is not to present a single methodology that will outperform all others already existing in academia and industry. Also, this research does not seek to identify a single price path for a given commodity and forecast an accurate projection for the future. Instead, price forecasting intends to be performed for a wide spectrum of market scenarios for mine valuation purposes. Instead, the generation of prices, which can be applied in a model that simulates mine valuation will serve as a basis to identify consequences of a given market scenario in mineral asset valuation. The methodology is similar to the one proposed by Zhang, Nieto and Kleit [Zhang et al., 2015], and will rely on simulation and generation of several different paths for analysis. SDE's will be used to simulate price paths for nickel and cobalt.

Price trend and volatility will be the evaluated in this stage. Distinct future prices for the two commodities in study, based on historical data provided by USGS [USGS, 2015], will generated here. Computer simulation will be applied and used as the means of data storage, forecast generation and subsequent use for mine valuation. MATLAB was selected as the platform for this project. The platform contains a Financial Toolbox, which corresponds to a series of functions for mathematical modeling and statistical analysis of financial data.

MATLAB has been selected for use because it is an easily available software in the academic environment, and many studies have already been done in this tool. In addition, it contains a matrix structure that proves to be ideal for the study to be performed, particularly for the use of loops and iterations. The platform also contains both stochastic differential equation models inside its functions. Finally, the plotting and graphing tools are of extreme importance for visualization of the parameters in study.

\subsection{Stochastic Differential Equation Modeling}

The number of different models available in academia and industry for price forecasting is substantial. MATLAB's Financial Toolbox [Mathworks, 2016b] describes a total of seven different models for price forecasting, and these can be grouped into two categories: random drift and mean-reverting drift. Therefore, the selection of two distinct methodologies was considered in order to encompass a wider spectrum of possible alternatives for commodity price forecasting. The two selected models 
were the following:

1. Hull-White/Vasicek (HWV) Gaussian Diffusion model (also referred in this thesis as "HWV" model or Mean-Reverting model)

2. Geometric Brownian Motion (GBM) model

The use of these models in particular was firstly based on the recognition of meanreverting models accurately behaving as historical prices for commodities. Secondly, even though the random drift is not completely recognized in academia as the most accurate prediction procedure for a commodity price, is certainly a good means to obtain a wide spectrum of price scenarios to be generated and evaluated with the dynamic mineral asset valuation model.

Pindyck [Pindyck, 1999,Pindyck, 2001] recognizes the superiority of mean-reverting models over geometric brownian motion, as expressed in a study done by Postali and Pichetti [Postali and Picchetti, 2006]. These researchers establish the relationship between supply/demand and the commodity prices, describing how lower prices usually banish less efficient producers, generating a market controlled by bigger players who raise the commodity price with less competition. Consequently, smaller players are able to enter the market, generating higher supply and consequently decreasing the prices. In this case, there is a reversion to the mean, due to the principle that the prices vary along a target value.

\subsubsection{Geometric Brownian Motion}

The formulation for the GBM model is given by:

$$
d X_{t}=\mu(t) X_{t} d t+D\left(t, X_{t}\right)+V(t) d W_{t}
$$

where:

- $X_{t}$ is the vector that contains the parameter of interest (the commodity price) 
- $\mu$ is the expected mean of the forecast, expressed as a vector of means over time

- $D$ is diagonal matrix, in which each element on the main diagonal is the corresponding element of $\mathrm{Xt}$

- $V$ is the parameter that controls the volatility rate

- $d W_{t}$ is a Brownian motion vector (responsible for stochastic movement)

The command for declaring and generating a GBM model file in Matlab is the following:

$$
G B M=g b m(\text { Return, Sigma })
$$

where:

- Return corresponds to the expected mean of the forecast

- Sigma corresponds to the standard deviation of the data input for analysis

Given the exponential form of the GBM model, prices in this case were expressed as year to year percent change of the log price value. The "Return" parameter corresponds to the commodity price mean. The sigma parameter, in the same fashion, corresponds to the standard deviation of the relative percentual change of prices expressed by their logarithm values. A simple representation of how the generation of this model is performed can be visualized in Table 3.1 and Figure 3.1.

Rate of change (in percentage) is the ultimate factor extracted from the historical data, which will base future prices on the pace of changing prices in the historical data. The starting point for the forecasts can either be the last historical price value or the overall mean of the historical data.

The resultant GBM model will be used to simulate a specified number of price forecasts. The model is stored on a MATLAB file. 
Table 3.1: Nickel price log differentiation

\begin{tabular}{|c|c|c|c|}
\hline Year & Ni Price (US\$/tonne) & Log10 Price & Rate of change (\%) \\
\hline 2000 & 8640 & 3.937 & - \\
\hline 2001 & 5950 & 3.775 & $-4.12 \%$ \\
\hline 2002 & 6770 & 3.831 & $1.49 \%$ \\
\hline 2003 & 9630 & 3.984 & $4.00 \%$ \\
\hline 2004 & 13800 & 4.140 & $3.92 \%$ \\
\hline 2005 & 14700 & 4.167 & $0.66 \%$ \\
\hline 2006 & 24200 & 4.384 & $5.20 \%$ \\
\hline 2007 & 37200 & 4.571 & $4.26 \%$ \\
\hline 2008 & 21100 & 4.324 & $-5.39 \%$ \\
\hline 2009 & 14600 & 4.164 & $-3.70 \%$ \\
\hline 2010 & 21800 & 4.338 & $4.18 \%$ \\
\hline 2011 & 22890 & 4.360 & $0.49 \%$ \\
\hline 2012 & 17530 & 4.244 & $-2.66 \%$ \\
\hline
\end{tabular}

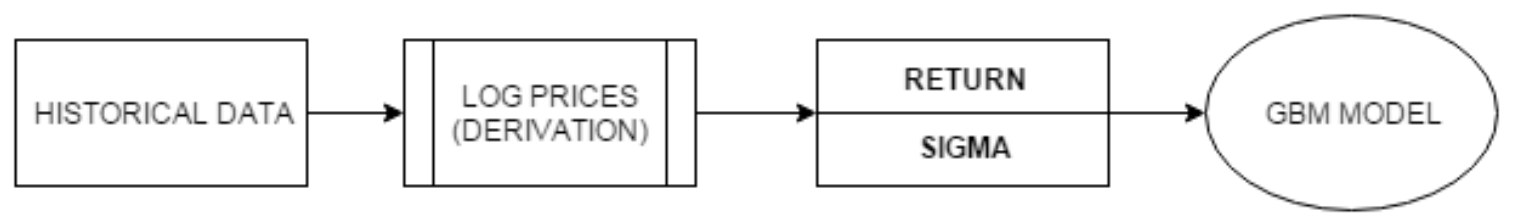

Figure 3.1: Geometric Brownian Motion model generation

\subsubsection{Mean-Reverting}

In the same fashion, the formulation for the HWV model is presented as follows:

$$
d X_{t}=S(t)\left[L(t)-X_{t}\right] d t+V_{t} d W_{t}
$$

- $X_{t}$ is the vector that contains the parameter of interest (the commodity price)

- $S$ is the rate of reversion to the mean

- $L$ is a vector that holds the mean of values to be used in each increment of forecast

- $V$ is the parameter that controls the volatility rate 
- $d W_{t}$ is a Brownian motion vector (responsible for stochastic movement)

The command for declaring and generating an HWV model file in Matlab is the following:

$$
H W V=h w v(\text { Speed, Level, Sigma })
$$

Unlike geometric brownian motion, this model contains three variables. Level corresponds to the mean level of a forecast at a given time, and is equivalent to the "Return" parameter of the geometric brownian motion. Sigma is equivalent in both models, and represents the standard deviation that will be applied to a price forecast, as a way to express the volatility of the future prices. Finally, the "Speed" parameter corresponds to the the rate in which a parameter reverts to the mean. Higher speed values show that, once the forecast starts, the values will reach the mean value at a faster pace.

These parameters are calculated through linear regression of prior data. The regression line represents the targeted mean of future prices, while the linear coeffient of the line corresponds to the "Speed" parameter value. A visualization of the linear regression procedure for nickel historical data ranging from 1980 to 2012 can be seen in Figure 3.2. Finally, a summary of the HWV model generation is expressed in Figure 3.3.

\subsection{Simulation of Price Forecasts}

Simulation is the standard methodology to generate different price forecasts. A Monte Carlo simulation methodology was established, following recommendations of work published by Nieto and Zhang [Nieto and Zhang, 2013] and Willingham [Willingham, 2013], The MATLAB software is also available to perform Monte Carlo simulation of pre-established SDE models, through the use of the Econometris Toolbox. The general command and structure to develop a Monte Carlo Simulation on the MATLAB interface is the following:

$$
[Y, E, V]=\operatorname{simulate}(\text { Model, numObs, Name, Value })
$$




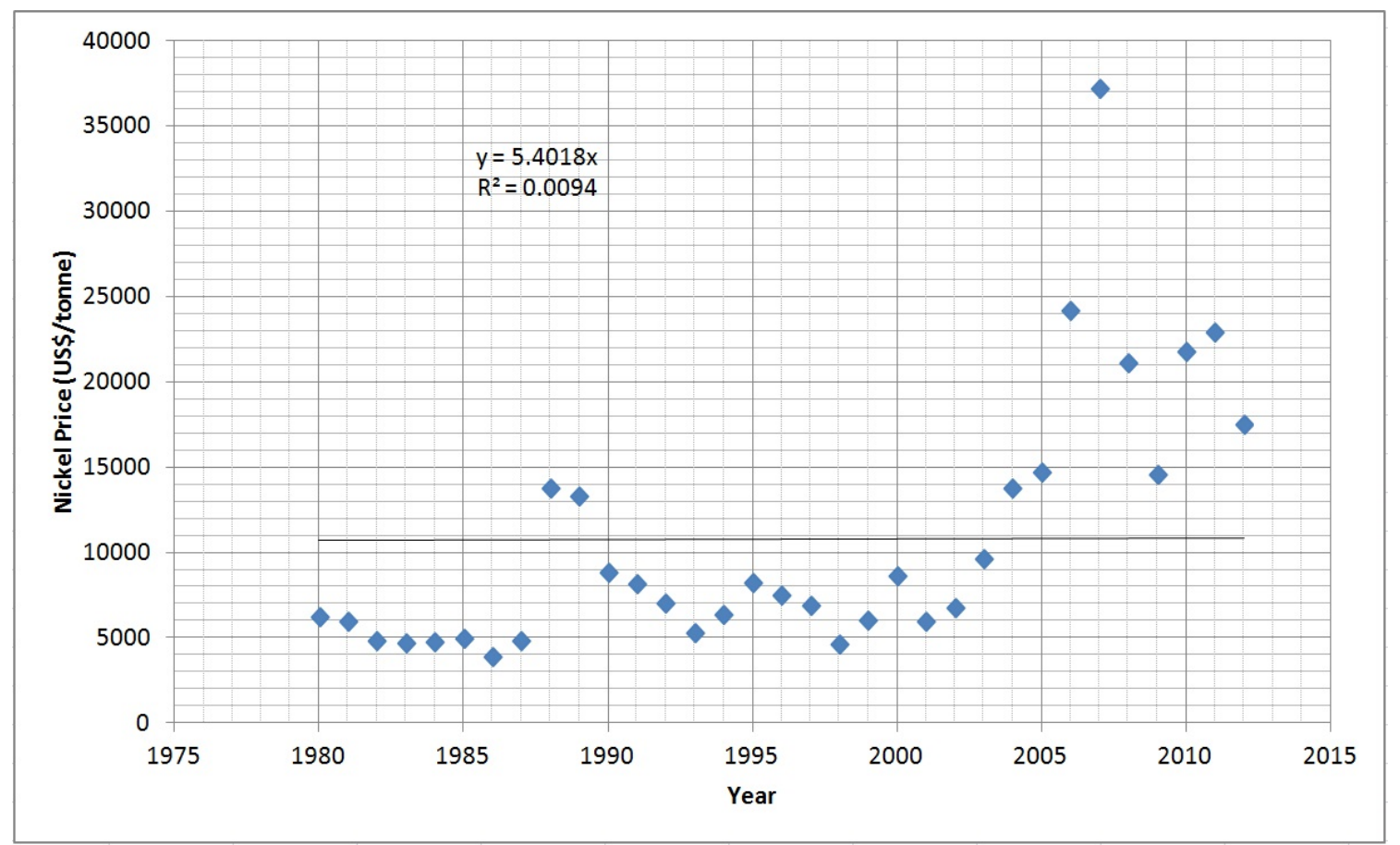

Figure 3.2: Linear Regression for Hull-White/Vasicek model determination

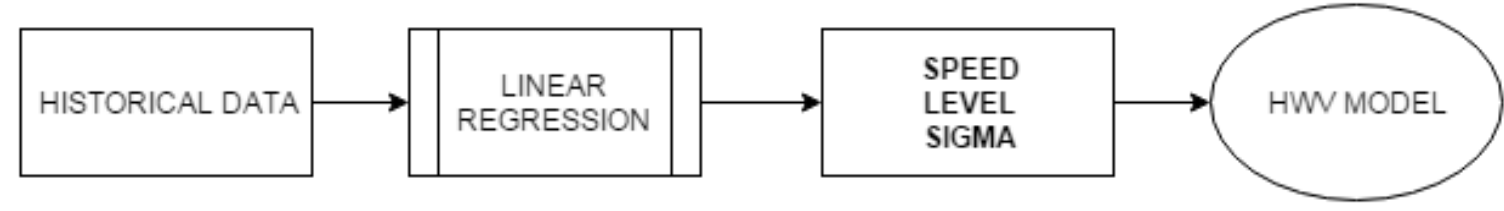

Figure 3.3: Hull-White/Vasicek model generation

where:

- $Y$ is the matrix of simulated data

- $E$ corresponds to a matrix of simulated mean zero innovations, also referred as increments (optional)

- $V$ corrresponds to a matrix of simulated of simulated conditional variance of the innovations in $\mathrm{E}$ (optional) 
- Model corresponds to the model generated during the price forecasting SDE modeling

- numObs corresponds to the number of simulations generated, which can be input by the user

- Name and Value correspond to different categories of simulations which can also be defined by the user, for different purposes or simulation categories

An example of how the simulation works can be visualized in Figure 3.4. A total of 10 different paths were generated, five of each using a different SDE model (GBM in blue and HWV in black).

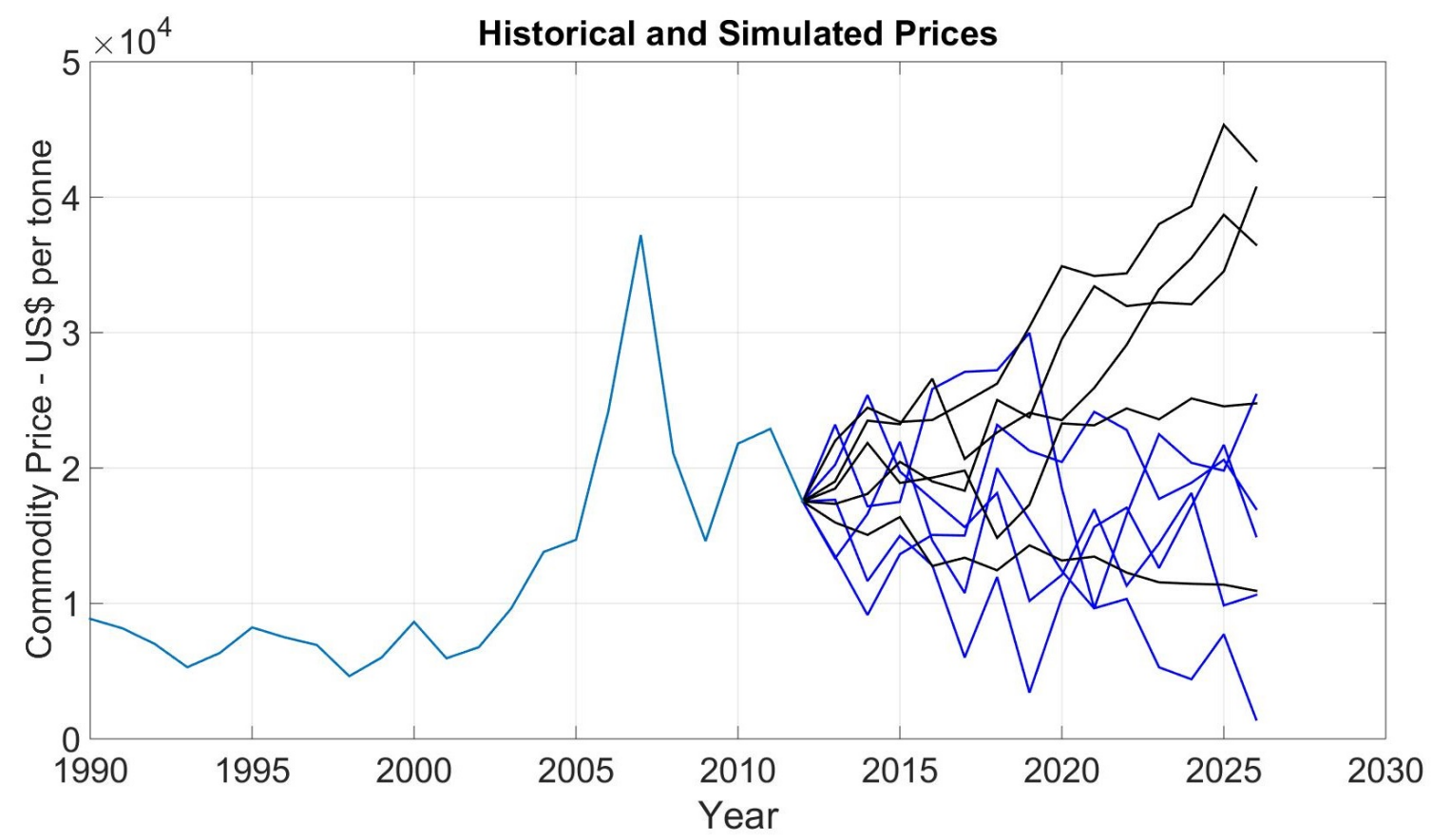

Figure 3.4: Simulation example

\subsubsection{Number of simulations required}

In order to determine the appropriate number of simulations, the main objective was to establish a number of simulations that would allow the modeling of a price 
scenario under specific circumstances of price trend and volatility. Considering these two restrictions, obtaining commodity prices with stabilized values of mean and standard deviation was targeted. The theory presented by authors such as Driels and Shin [Driels and Shin, 2004] and Ritter et al. [Ritter et al., 2011] demonstrated that the optimal number of simulations can be obtained by identifying a maximum target of simulations, based on the available computational power and balance of cost and time to generate these simulations.

Statistical significance was also applied for validation, using the methodology of identifying the minimum sample required in a population [Driels and Shin, 2004], with an approximation of the population's mean and standard deviation. The target of simulations was set at 1000, and the following formula was used to obtain the minimum number of simulations required for price forecasting:

$$
R>\left(\frac{z_{1-\alpha / 2} \cdot s_{0}}{\varepsilon \cdot x_{0}}\right)
$$

where:

- $\alpha$ corresponds to the significance level at 0.05

- $\varepsilon$ corresponds to the standard error set at 0.05

- $x_{0}$ and $s_{0}$ correspond to the mean and standard deviation of price forecasts at $\mathrm{R}=1000$ simulations, respectively

The following parameters specify the identification of the minimum number of simulations required, considering a 95\% confidence and $2.5 \%$ standard deviation from the targeted values. In order to obtain an accurate prediction for the whole spectrum of price scenarios analyzed, this analysis was performed for all possible values of price mean and volatility, as well as the two different SDE models used in this research project. The definition of different models, means and price volatility trends are the following:

- Price Forecasting model: GBM and HWV

- Volatility: 1 sigma and 1.25 sigma 
- Commodity: Nickel and cobalt

Using Equation 3.6, the minimum number was calculated for the different factors in the study. Table 3.2 represents the minimum number of required simulations according to the number of years simulated on the price forecast, targeting a maximum of eleven operating years for the mine valuation methodology proposed and developed in the project.

Table 3.2: Number of simulations required

\begin{tabular}{ccc}
\hline Scenario & Mean-Reverting (HWV) & Geometric Brownian Motion (GBM) \\
\hline $1 \sigma-$ Nickel & 20 & 12 \\
$1 \sigma-$ Cobalt & 28 & 12 \\
$1.25 \sigma-$ Nickel & 27 & 15 \\
$1.25 \sigma-$ Cobalt & 47 & 15 \\
\hline
\end{tabular}

The highest number of simulations required, according to the historical data input in the algorithm, corresponds to a total of 47 . Therefore, the number of simulations for each simulated price scenario was set at 50 .

Finally, an alternative technique was used to cross-validate the required number of simulations. A total of 1000 simulations of price forecasts were generated using 11 years as the default value for each iteration. Then, the minimum number of simulations required was obtained bycalculating the ratio of a mean price divided by the previous price considering one less simulation. The principle of rolling averages was applied in this context.

$$
\frac{\mathrm{d}\left(\mu_{i}-\mu_{i-1}\right)}{\mathrm{d} x}<0.01
$$

The maximum ratio between the rolling average of two prices could not exceed a difference of 0.01 . The optimal number of iterations using this methodology ranged from 37 to 43 after several simulations were run. Consequently, the desired number of $\mathrm{R}=50$ was validated to be the appropriate number of simulations applied to the methodology of price forecast generation proposed on the research. 


\subsection{Methodology}

Price forecasts were determined using different levels of mean and volatility as input parameters. The generation of the forecasts consisted of the creation of a computer algorithm based on MATLAB which would generate future commodity prices according to the desired market scenarios to be evaluated on the mine valuation model. Table 3.3 summarizes the modification parameters on the SDE models that were used to generate variability in prices.

Table 3.3: SDE model modifying parameters

\begin{tabular}{lcc}
\hline Parameter & Geometric Brownian Motion (GBM) & Mean-Revering (HWV) \\
\hline Price Trend & Return & Level \\
Volatility & Sigma & Sigma \\
\hline
\end{tabular}

The default process of price forecasting generates simulatons that possess a characteristic mean and standard deviation, based partially on the historical data and the forecasted values. "Return" and "Level" specify the mean of the historical data to be input and Sigma represents the snatdard deviation of the simulations ready to be generated.

These parameters were manipulated by using modifying factors multiplying the default values of mean and standard deviation, according to the formulation below:

$$
\begin{aligned}
& \sigma_{\text {new }}=\sigma_{\text {historical }} \cdot M_{\text {factor }} \\
& \mu_{\text {new }}=\mu_{\text {historical }} \cdot M_{\text {factor }}
\end{aligned}
$$

The modifying factor was set up to account for different price scenarios and simulate a wide variety of price forecasts. For this research project, the following values were selected:

- Modifying factors for the mean: 0.80 (low trend) and 1.20 (high trend) 
- Modifying factors for the standard deviation: 1.00 (default) and 1.25 (high)

The reason why lower values of modifying factor for volatility were not selected for the modeling was because, during the development of the project, manipulating volatilty to values considered lower than the natural one presented by the historical prices generated forecasts very similar in mean values, with small variation in regard to standard deviation. Therefore, the decision was to use inherent and high values of standard deviation to generate price scenarios for mine valuation modeling.

Also, by selecting higher values of volatility, some simulations produced negative price values. This abnormality only occurred in extreme volatility, and these specific price trends were discarded from analysis.

\subsubsection{Beta index determination}

The methodology to correlate Beta values with the sigma parameter on the price forecasting models followed the methodology developed by Noble [Noble, 2014]. By using the same commodity prices as described by the researcher, proportionality between the sigma parameter and the beta index was established, considering that $\beta$ is directly proportional to the standard deviation of the commodity in study. The year 1980 was selected as the preferred year for the start of historical data analysis, because the data was not adjusted for inflation and selecting previous periods could cause distortion in price forecasts. The values were extracted from the USGS database of mineral prices. The $\beta$ parameter was observed from years ranging from 1980 to 2012, with the following values colected:

- Nickel $\beta$ : 2.39 (1980 to 2012)

- Cobalt $\beta$ : 1.26 (1980 to 2012)

The observed values show that nickel has a higher volatility in the commodity market, compared to cobalt. Besides that, the modifying factors presented previously allow the identification of Beta values that will be input on the mine valuation model. They are represented in Table 3.4. 
Table 3.4: Beta values for Nickel and Cobalt

\begin{tabular}{ccc}
\hline Parameter & Regular (Inherent) value & High value \\
\hline Nickel & 2.39 & 3.00 \\
Cobalt & 1.26 & 1.58 \\
\hline
\end{tabular}

\subsubsection{Price sorting, validation and plotting}

After generation of price forecasts, the values were sorted in a matrix structure. Final validation was performed by calculating the mean and standard deviation of the generated prices, and checking if their output values correspond to the input parameters specified. Finally, the generated values were plotted for visualization by the user.

An overview of the price forecasting methodology is presented in Table 3.5.

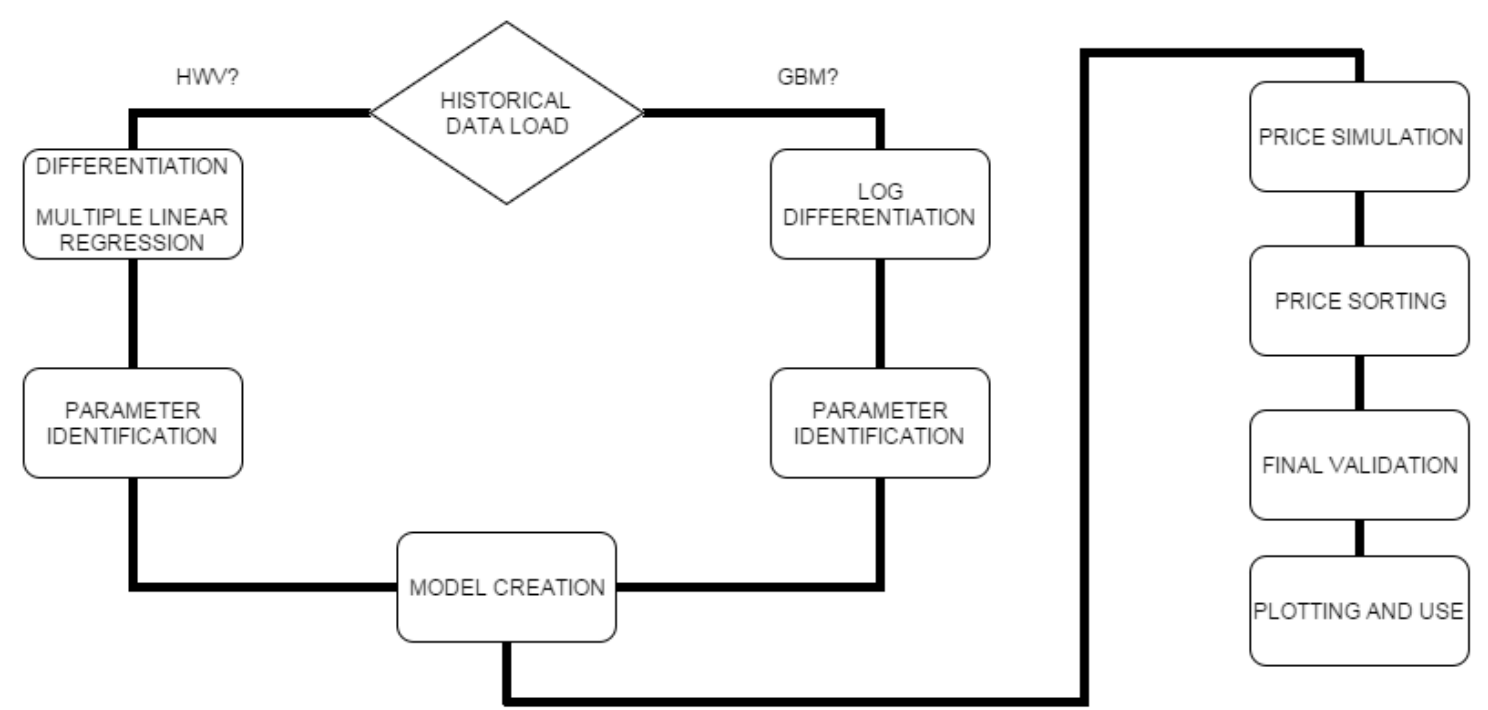

Figure 3.5: Price forecasting overview 


\subsubsection{Experiment Design}

Table 3.5 shows an experiment design with the price forecasts to be generated and subsequently used on the mine valuation modeling part of this research project. It specifies a total of 16 scenarios, generated through the combination of 4 factors with 2 levels each.

Table 3.5: Experiment Design

\begin{tabular}{lllll}
\hline Scenario & Forecasting Type & Volatility & Nickel Price Trend & Cobalt Price Trend \\
\hline 1 & HWV & Regular & Low & Low \\
2 & HWV & Regular & Low & High \\
3 & HWV & Regular & High & Low \\
4 & HWV & Regular & High & High \\
5 & HWV & High & Low & Low \\
6 & HWV & High & Low & High \\
7 & HWV & High & High & Low \\
8 & HWV & High & High & High \\
9 & GBM & Regular & Low & Low \\
10 & GBM & Regular & Low & High \\
11 & GBM & Regular & High & Low \\
12 & GBM & Regular & High & High \\
13 & GBM & High & Low & Low \\
14 & GBM & High & Low & High \\
15 & GBM & High & High & Low \\
16 & GBM & High & High & High \\
\hline
\end{tabular}

\subsection{Results}

A total of 1600 simulations were performed, divided into two different commodity categories, and 16 price scenarios for each commodity, with 50 simulations for each scenario. A total of 11 years were used as the total number of steps for simulation, taking in consideration that a 10-year operation proposed in the mine valuation methodology may require additional years to conclude the mining, milling and material sales. 
The price forecasting input parameters are the following:

- Commodity Price Trends: 80\% (low) and 120\% (high), for modification of the Historical Mean

- Commodity Volatility: $100 \%$ and $125 \%$, for modification of the Historical Beta

- 2012 as Start Date, 11 years of simulation, 50 Iterations for each Scenario

- Historical Data to be considered: 1980 to 2012

For clarification, a price scenario corresponds to the combination of both nickel and cobalt price simulations compiled together into one single file, for posterior use. Therefore, some scenarios that contain repeated parameters such as a commodity price trend (e.g.: Scenarios 1 and 2 contain "Low Ni" as a parameter) contain forecasts that were calculated repeatedly. This process was selected to facilitate the simulation procedure, as well as allow a standard for comparison of price forecasts, in terms of informal validation. However, repeated price forecasts will not be presented as graphs in this document.

Nickel prices are represented in Figure 3.6. The left column represents scenarios of pessimistic forecasts, while the others show optimistic projections for prices. The four plots on the upper part represent the mean-reverting model applied to the historical data. Different levels of volatility are applied, as observed by the wider range of the plots with 1.25 Sigma.

Optimistic future prices do not necessarily have upward trend in the mean-reverting model. Nickel was in a natural ascending trajectory according to the historical data, therefore the optimistic approach allowed values to be maintained on a higher level, compared to the historical data mean (shown as the black line in all the plots). The geometric brownian motion scenarios for nickel are also presented in Figure 3.6. The starting values differ from the ones in the mean-reverting model, because modifying the mean on the GBM model requires bringing the initial value of forecasts to the desired mean value of forecasts. Geometric brownian motion, unlike the mean-reverting model, constains randrom drift.

For cobalt prices, the same methodology shown and explained for nickel is applied presented in Figure 3.7 .Each scenario contains certain specifications of price trend 

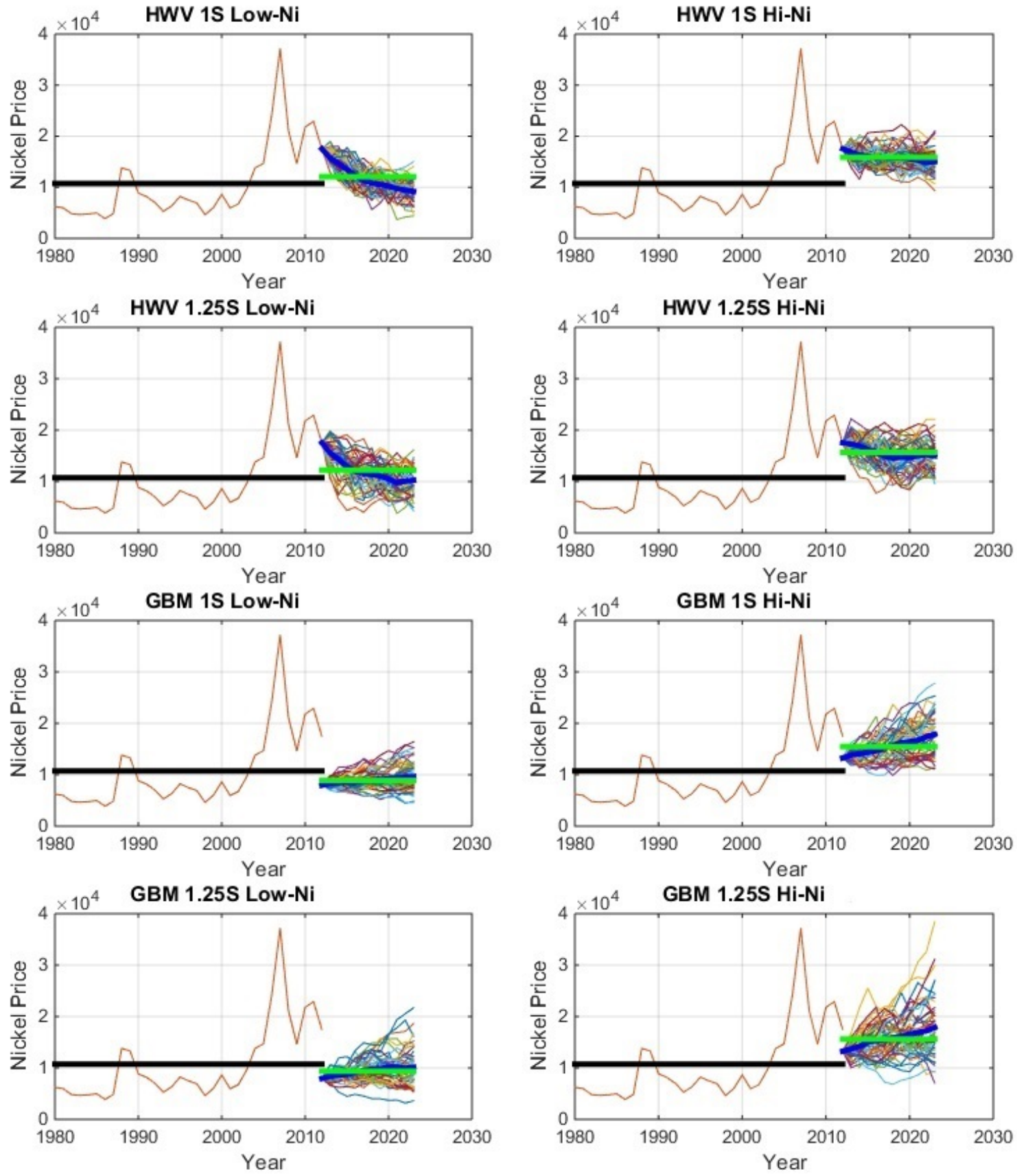

Figure 3.6: Nickel price forecasts 

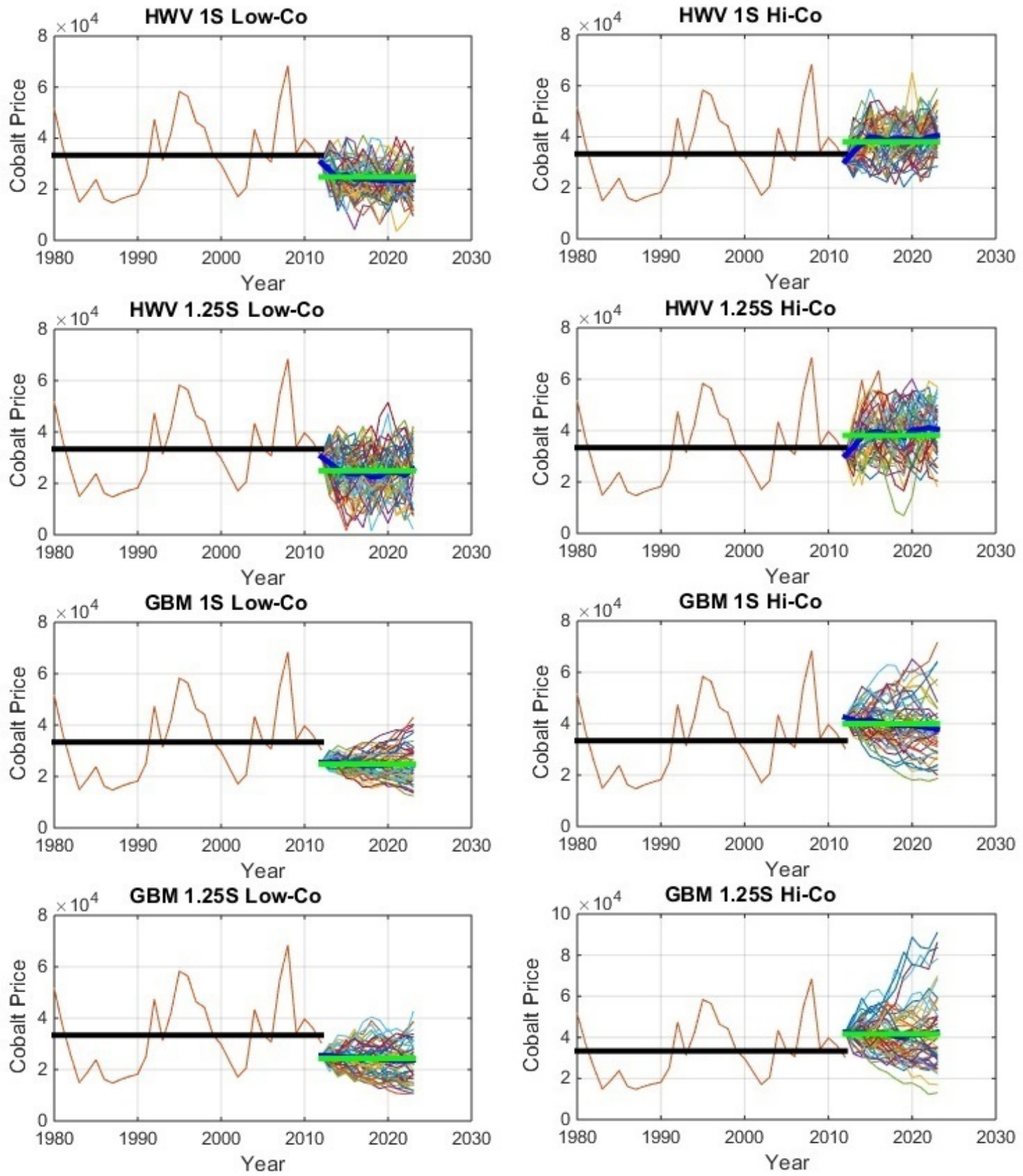

Figure 3.7: Cobalt price forecasts 
and volatility, according to the parameters proposed on the experiment design. However, the relative changes in values in these simulations are smaller compared to the ones visualized for nickel. Cobalt's natural volatility (1.26 in Beta index) is lower than nickel and its intrinsic volatility of 2.39 .

Price forecasts generated with the GBM model do not present the same disparity of initial values compared to the HWV model. Historical data for the commodity possesses less variability and, at the initial time of the forecast start (year 2012), the last observed value is very close to the overall historical data mean. Therefore, the progression of values in the very first year is more natural.

\subsection{Summary and Conclusions}

This chapter presented a methodology for price forecasting based on stochastic differential equations. One of the facts observed by simulating prices with extreme levels of volatility (identified in this context as prices with over $50 \%$ more volatility than default values calculated by the historical data) was the possible generation of negative price values. The models do not distinguish between positive and negative values while generating forecasts.

The number of simulations was determined using statistical analysis on the mean and standard deviation of the generated prices. The proposed algorithm identified an optimum solution in values ranging from 37 to 47 simulations for each price scenario, as a minimum requirement to obtain stabilization of mean and standard deviation. A value of 50 simulations was subsequently selected as the default value.

In addition, the mean-reverting drift model allows for more control of simulated paths in forecasting. This conclusion goes in favor with the observations performed by other researchers [Pindyck, 2001, Postali and Picchetti, 2006], regarding the use of mean-reverting drift models instead of random drift ones. The proposed algorithm was able to simulate price forecasts in the whole desired spectrum of commodity future price trends. These prices will have significant impact on mineral valuation for the hypothetical project presented in the subsequent chapters. 


\section{Chapter 4}

\section{Computer Modeling for Mine Valua- tion}

Computer applications are widely used for the development of mineral asset valuation, focusing on financial methods to evaluate the economic value of a mining project. The literature review has gone through theoretical aspects of mine valuation in detail, therefore this chapter will describe the approach used in this study.

The existence of various mine valuation methods is justified by the principle that mineral assets vary in stages of exploration, development and discovery. The proper solution for determining the value of the asset must then be selected by the individual performing the task. Nevertheless, consistency exists with the use of methodologies such as a discounted cash flow approach, capital and operating cost structure, use of present value for valuation and significant sensitivity with prices input on the system. This chapter will discuss the available solutions for mineral valuation, and present a new methodology of mineral valuation based on optimization techniques.

\subsection{Discounted Cash Flow Model}

Discounted cash flow considers the value of a given mineral reousrce or deposit as if the whole value could be obtained from it in the exact present moment. In order to obtain this value, several technical and economical details must be considered. A feasibility study will determine the amount of material that should be extracted, processed, refined and eventually sold in order to generate revenue. All these processes contain intrinsic costs associated with that. The overall determination of the 
actual profit for mining and developing the subsequent processes must take all these parameters in consideration.

Finally, taking in consideration factors such as inflation, taxes and most importantly a discount rate (the rate in which the money will lose value along time), it is possible to calculate the value of a given mineral project. Other economic parameters, such as inflation and taxes must be considered, and finally the value of the project can be calculated. The figure below shows an example of a discounted cash flow spreadsheet model. The material produced by the mine will be sold and generate value on the long run, which will then be converted to how much value it possesses in the present.

\begin{tabular}{|l|l|r|r|r|r|r|}
\hline \multirow{2}{*}{ Parameter } & \multirow{2}{*}{ UNIT } & \multicolumn{5}{|c|}{ YEAR } \\
\cline { 3 - 7 } & & 1 & 2 & \multicolumn{1}{c|}{3} & \multicolumn{1}{c|}{5} \\
\hline Yearly throughput & Tons & $20,000,000$ & $20,000,000$ & $20,000,000$ & $20,000,000$ & $20,000,000$ \\
\hline Grade & $\%$ & $1.5 \%$ & $1.5 \%$ & $1.5 \%$ & $1.5 \%$ & $1.5 \%$ \\
\hline Recovery & $\%$ & $92.0 \%$ & $92.0 \%$ & $92.0 \%$ & $92.0 \%$ & $92.0 \%$ \\
\hline Commodity Price & $\$$ /ton & 13845 & 13845 & 13845 & 13845 & 13845 \\
\hline Discount Rate & $\%$ & $10 \%$ & $10 \%$ & $10 \%$ & $10 \%$ & $10 \%$ \\
\hline Capital Cost & $\$$ & $(4,800,000.00)$ & & & & \\
\hline Operating Cost & $\$$ & $(2,140,000,000)$ & $(2,140,000,000)$ & $(2,140,000,000)$ & $(2,140,000,000)$ & $(2,140,000,000)$ \\
\hline Revenue & $\$$ & $3,821,220,000$ & $3,473,836,364$ & $3,158,033,058$ & $2,870,939,144$ & $2,609,944,676$ \\
\hline NPV & $\$$ & $4,422,916,987.23$ & & & & \\
\hline
\end{tabular}

Figure 4.1: Discounted Cash Flow Model

\subsubsection{Overview}

Discounted cash flow (DCF) analysis is a methodology for financial evaluation used in every type of economic setting, in order to obtain economic an estimation of the value in a given project, operation or purchase. It is usually applicabe for projects or proposals in which there is accurate description and determination of costs associated with producing and selling a product.

If the costs and estimates of sales are not well established, the result of a net present value estimation will be insufficient. Therefore, early stage projects must be analyzed very carefully through this methodology. Since the value of a mineral asset corresponds to the present value of cash flows that this asset is able to generate over 
the total time of its operation, it is necessary to understand tje impact of commodity price and ore grade for generation of revenue.

Considering that fixed costs usually do not change over time, the most significant impact on the NPV of a project will be the main drivers of revenue generation: price and grade. Ore grade is the parameter that determines the amount of economic value that every unit of material (whether it is in tonnes, ounces or grams) holds, while price corresponds to how much the buyer will pay for the valuable content.

\subsubsection{Mechanics}

Mineral extraction planning allows the determination of the amount of material that will be mined, processed, refined and eventually sold. The final product will be sold for a specific price after it has been produced. Considering the operating costs associated to producing the commodity, a discounted cash flow analysis takes in consideration the tonnage and grade of the mineral reserve, and the product of the mineral processing and refining will generate a certain revenue.

Capital expenses, operating costs, and finally, taxes, inflation and mineral royalties may also be part of the identification of the economic value of a mineral project. These values represent forecasts, but can be added in order to generate a project NPV. The usual formuation for the net present value of a mining project is given in the following way:

$$
N P V=\sum_{n=0}^{N} C_{n} /(1+r)^{n}
$$

where:

- $N P V$ is the Net Present Value

- $C$ is the cash flow in period $n$

- $r$ is the discount rate

- $n$ is a specified time period (usually years) 


\subsubsection{Discount Rate}

The rate in which monetary value is lost as time progresses is one of the most crucial aspects of mine valuation. Selecting a different discount rate may cause more than $50 \%$ variation in the value placed on a project [Mohammad, 1997]. Discount rates usually range from $8 \%$ (very aggressive and optimistic) to $15 \%$ (more conservative). Optimistic discount rates are usually applied in the mineral exploration industry [MLM, 2015] in order to attract investors to greenfield projects, also taking in consideration that no machinery or infrastructure has been purchased, which will certainly have a decreased value over time.

Darling [Darling, 2013] specified the $10 \%$ to $15 \%$ range for discount rates for mineral asset valuation. For the purpose of this project, these two values will be tested in the mine valuation methodology proposed. Figure 4.2 shows the loss value in percentage for a given asset as time progresses, in years. The revenue generated by a mining operation five years in the future corresponds to only $60 \%$ of the value generated in the present. For the more pessismistic discount rate, such value only corresponds to approximately $50 \%$.

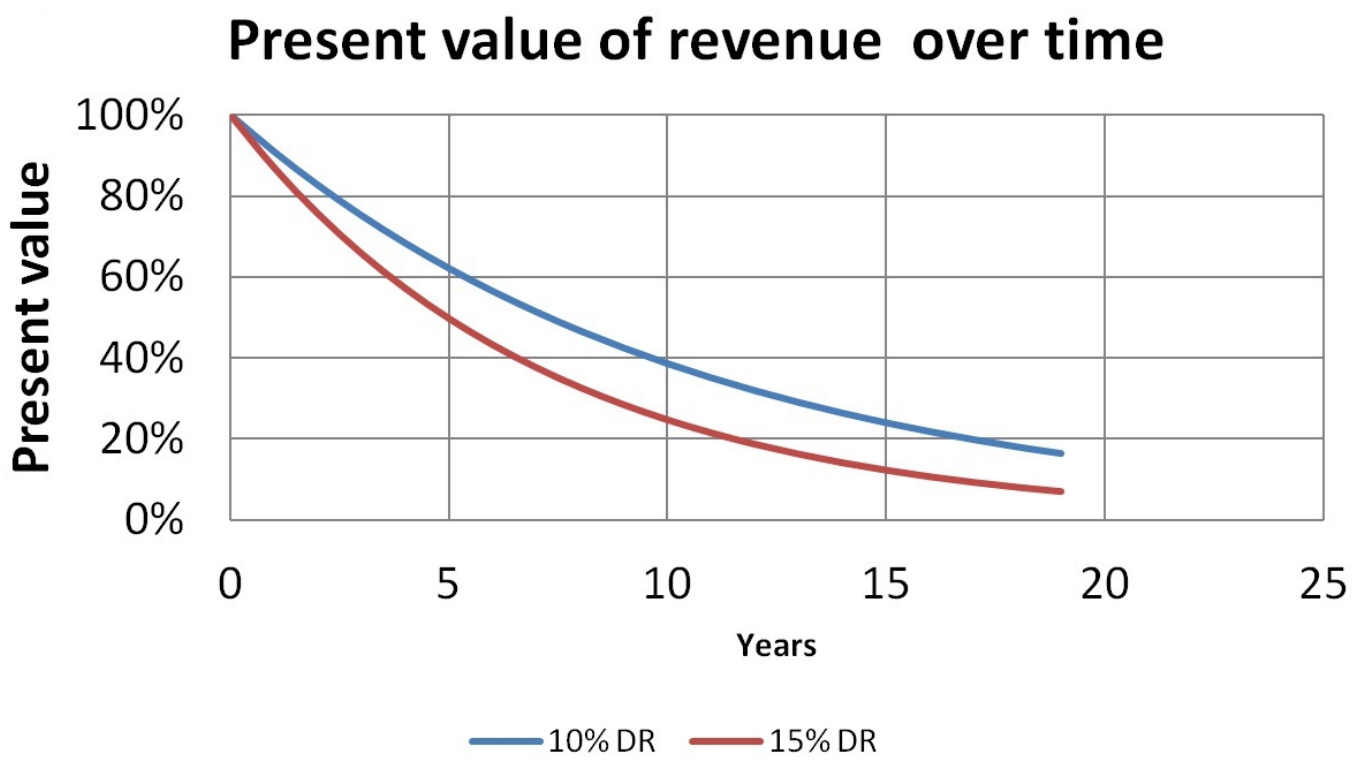

Figure 4.2: Present value of money for two discount rates 


\subsubsection{Revenue}

Revenue is primarily dictated by commodity price, and greatly influences the payback period of a mining project. Capital and operating costs are subtrated from the revenue generated in operation to calculate the profit on a specific period of time.

The following factors are the most impactful on a mining operation revenue:

1. Yearly throughput on the mine, mill or smelter

The throughput specifies how much material is being produced, therefore an estimate of how much material will be sold can be obtained. The main assumption in this case is that the entirety of the material produced can be sold.

2. Production capacity

This parameters specifies the default capacity of the operation based on design constraints. The production capacity corresponds to how much the operation is able to produce in its maximum design rate. It is usually done at static rates, with occasional ramping up in the initial stages of operation

3. Commodity metallurgical recovery

Recovery is a function of the mined ore grade and the final product grade. It is determined through previous metallurgical studies, extensive experimental testing and further scale-up.

\subsection{Real Options Valuation Model}

Real option valuation (ROV) corresponds to a relatively new method of valuation based on the Black-Scholes option pricing mode [Fischer Black, 1973]. It takes into consideration options such as expansion, shrinkage, interruption or permanenent shutdown (decomissioning) of a mining operation in order to identify the most viable economic scenatrio. The modifications are based on a deterministic approach, through the valuation of constant yearly increases or decreases in production. It relies on the creation of different operation scenarios, and subsequent testing of these scenarios through simulation. 


\subsubsection{Overview}

This methodology has its basis on the discounted cash flow analysis, because both share the principle of valuation through the use of established cash flows, taking into consideration discount rates. However, the real options approach applies managerial flexibility in the valuation of a project.

Flexibility corresponds to the ability of modifying production parameters in favor of producing higher revenue under a specific market condition. For instance, the ROV method suggests increase in production when commodity prices are high. This procedure is preferred because, according to the main factors that affect the revenue fo a mining project, the yearly throughput of a mining operation is the only parameter that can be modified in order to target operational flexibility.

Metallurgical recovery and design rate are instrinsic of the mined ore and the operation design, respectively. There is no possibility to increase metalurgical recovery without great investment in new technologies or more expenditure in general. Also, once a mine operation or processing plant is designed, it has to function under specified conditions. Therefore, operational flexibility targets modifcation of throughputs, and subsequently product generation, in order to obtain more revenue. The procedure to evaluate a project under the ROV methodology is in Figure 4.3

Under this methodology, a network of possible scenarios is created, and different branches of the network correspond to possible operational scenarios in which a mine or processing plant can run. Computer simulation will identify what is the preferable operational procedure, targeting NPV maximization.

\subsection{Dynamic Option Valuation Model}

This project proposed a new valuation model based on dynamic modeling of of mining projects. The approach is denominated Dynamic Option Valuation (DOV) and is fundamentally unique from from DCF and ROV methods because it proposes operational flexibility through the use of stochastic methods of optimization under economic uncertainty. The valuation procedure relies on input parameters for determination of the economic value of a mineral project. The parameters are described in the following way: 


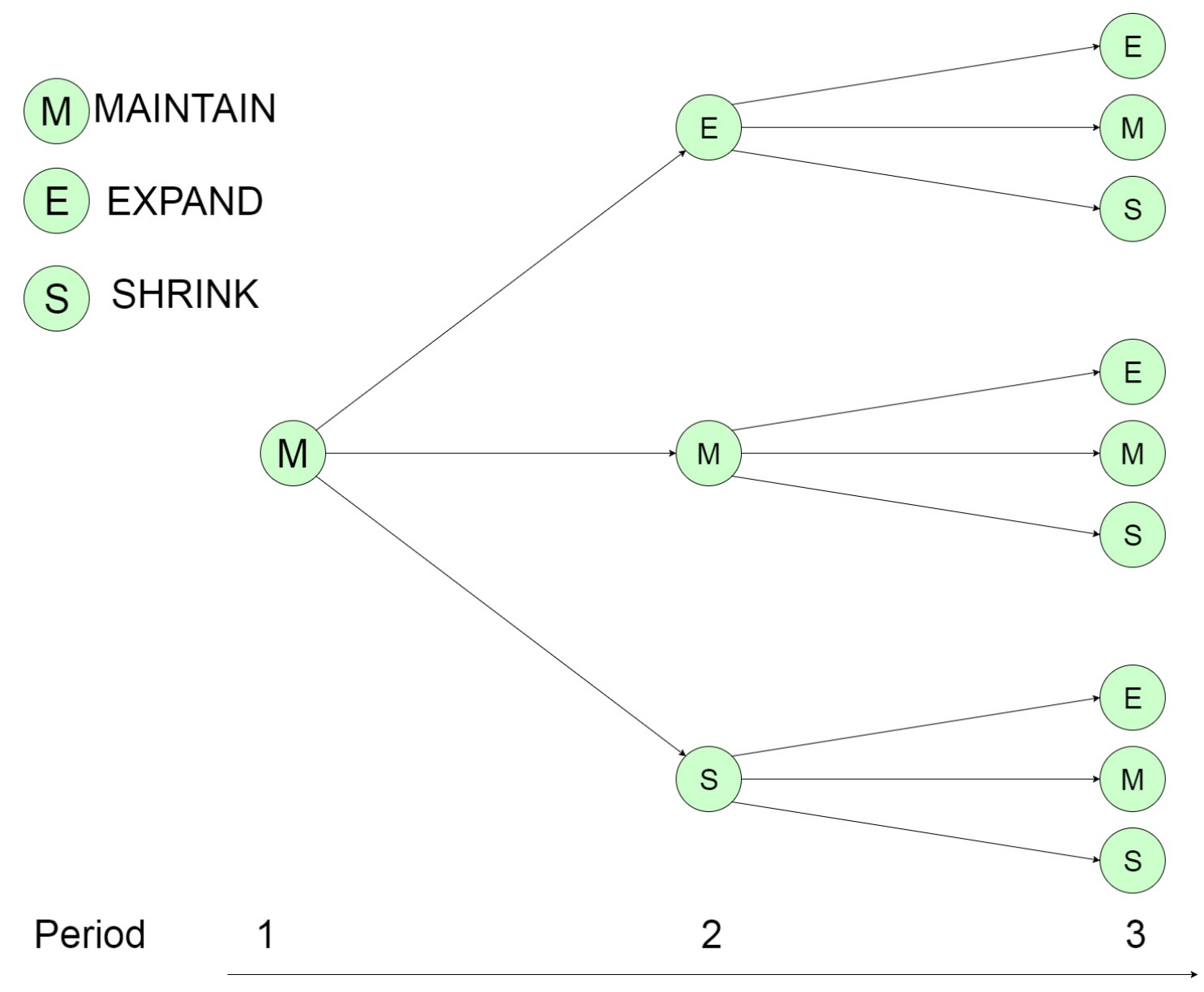

Figure 4.3: Real Option Valuation Model

- Fixed: Mineral grade, metallurgical recovery, discount rate, deposit size, number of simulations, number of operating years, stockpile maximum size

- Stochastic: Commodity prices

- Variable: Production capacity (mine, mill and smelter throughputs, sales rates), stockpile inventory

The DOV methodology is described in the following steps:

1. Generate DCF model with standard input parameters 
The standard mine valuation model is used for NPV calculation with static values for production and stockpile inventory. All the other input parameters remain the same, for comparison

2. Generate a single stochastic price forecast for model

The price forecast will be used to calculate revenue for the product generated by the operation, and subsequently the NPV of the mining project

3. Use an optimizer to select mine/mill/smelter throughput and sales rates

The optimizer will obtain optimal operating parameters. Buffer stockpiles are generated due to the offset of the values between adjacent processes (mine $>$ $\mathrm{mill} /$ smelter $>$ sales)

4. Repeat steps 2 and 3 for $n$ large number of price forecasts

The same process is repeated for as many price forecasts and scenarios are available

5. Aggregate and compare results

Compiling the generated results of both methods to compare increase in profit due to the use of DOV

\subsubsection{Overview}

Operational flexibility is generated by allowing the processes to work below or above their default capacity, using an optimization solution to identify the proper working capacity for higher profit generation. Figure 4.4 presents the stochastic operational flexibility described in this chapter. For instance, the mine is allowed to produce material between $+-5 \%$ of its rated capacity. The throughput values are selected by an optimizer inside the feasible region.

Maximizing the NPV of the mining project occurs when the optimizer searches and identifies the value which will generate the highest profit under that technical and economic context, combining production for all the years and processes. The same principle is applied to the processing plant and sales rates.

The dynamic option model relies almost exclusively on optimization methods for the identification of production rate parameters. Global optimization, for instance, 


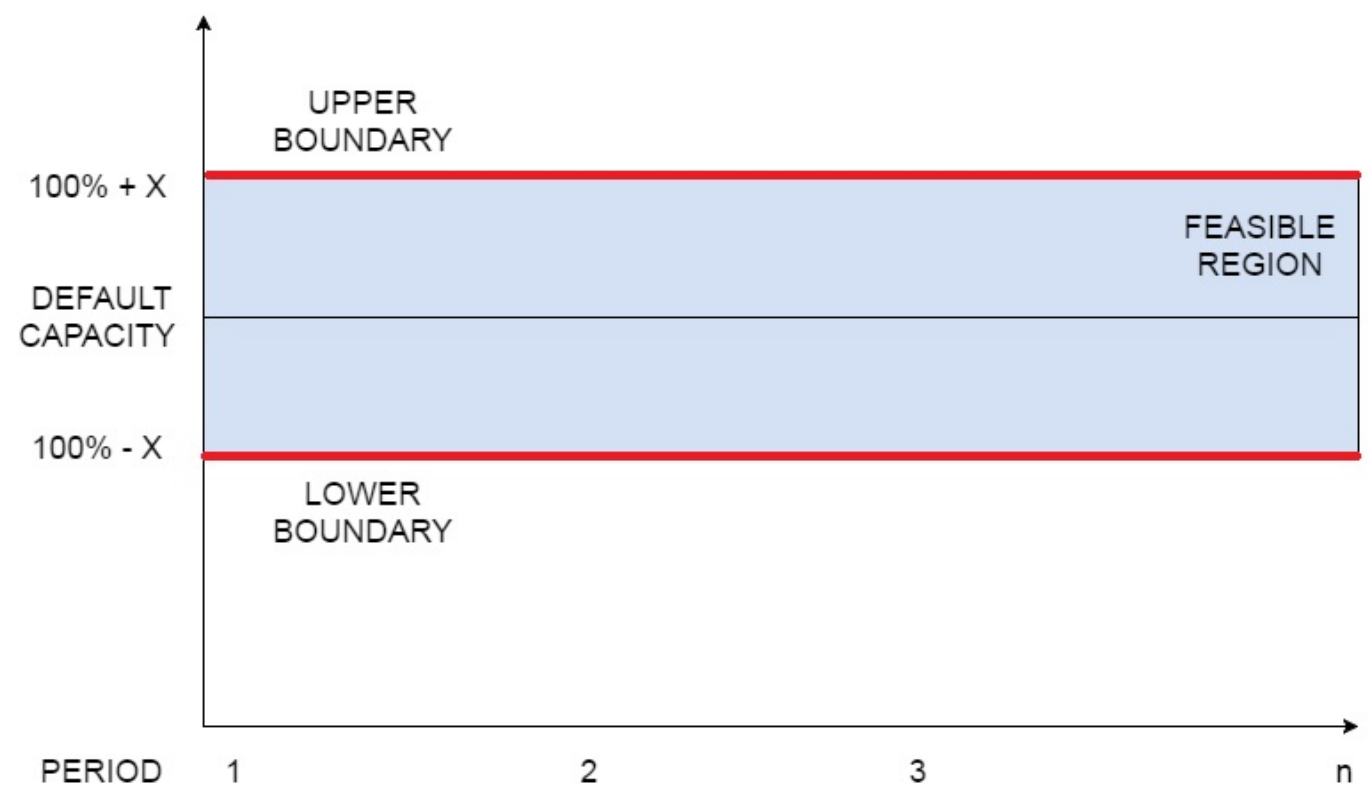

Figure 4.4: Dynamic option valuation - Feasible operating region

consists on the identification of optimal input parameters for a mathematical model, using a stochastic process. The input parameters of the model (contextualized in this scenario as the production rates for mine, mill and the sales rate for final product) are tested through a search algorithm, which outputs the values that will maximize the output parameter of choice.

In this case, the parameter to be optimized corresponds to the NPV of the mineral asset, in the same fashion as the ROV method. A stochastic approach is performed, instead of the usual deterministic methodology observed in the ROV and DCF methods. Unlike the traditional methods, the dynamic modeling methodology relies on subjecting the throughput parameters to uncertainty.

The offset between mine and mill production, for instance, may cause the following scenarios: miill throughput may be higher than mill throughput, on a given year; product sales may be higher than total material produced on a given year. In order to account for the discrepancy between mine, mill and sales rates, a buffer interface must be added to the model. In this case, the use of the raw stockpile will allow modifications on the parameters in question. Figure 4.5 presents the phenomena in question. 


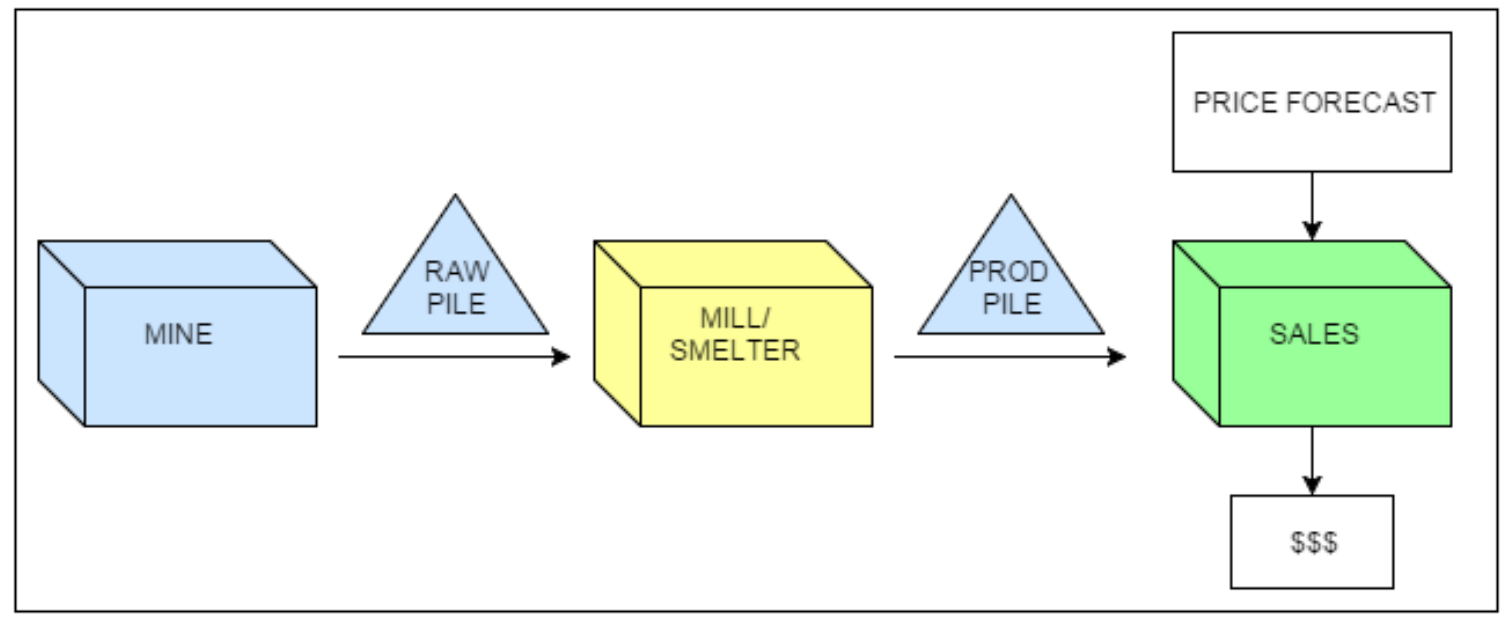

Figure 4.5: Buffer structures on the dynamic option valuation method

On the dynamic valuation model, the mill production rate is can be modified, including increase or decrease, in any value specified by the design of the operation. For instance, a mine design that allows the operation to run between $90 \%$ and $110 \%$ of the rated capacity could be selected for the dynamic option model. The processing plant, if allowed to participate on the same analysis, would be subjected to valuation under uncertainty on this aspect.

The mill is allowed to work between $+-15 \%$ of its rated capacity, and the buffer pile can grow or shrink as needed. As a result, the optimizer has chosen to increase and decrease mill throughput from the static value, based on the price forecasts. This strategy is used to account for possible situations in which the optimization has identified that establishing a bottleneck on the mill is preferrable to achieve more economic profit in the future. A visual representation of this process can be observed in Figure 4.6, that combines the dynamic mill and a buffer stockpile measurement over a period of 10 years.

\subsection{Application example}

A simple example was used to compare DOV to the standard methods (DCF and ROV). This study was performed to evaluate the validity of the proposed DOV in mineral valuation. A hypothetical nickel operation is presented for evaluation, based 


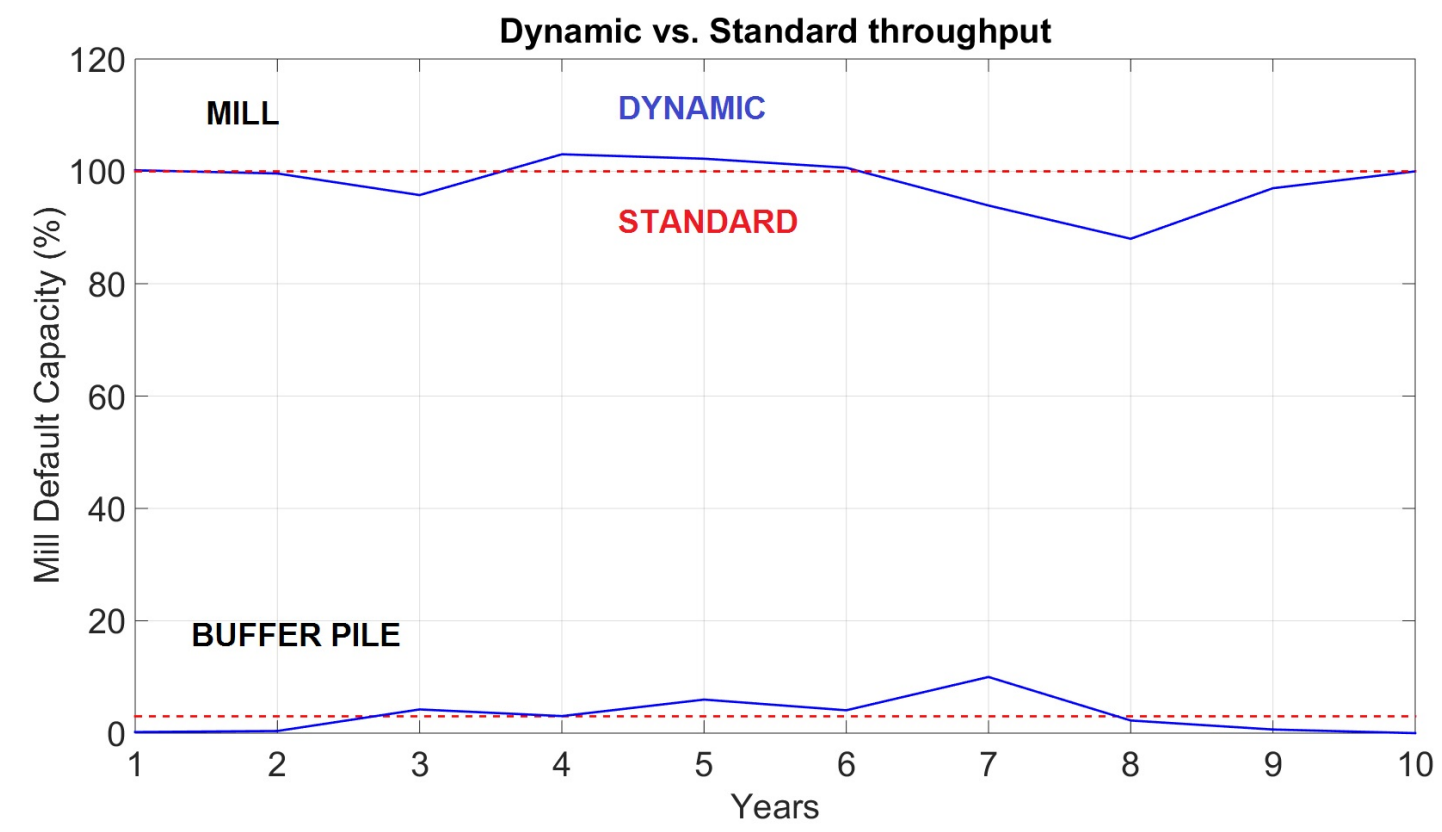

Figure 4.6: Standard vs Dynamic mill throughput example

on publicly available data. The data in question corresponds to one of the deposits presented by Metallica Minerals LTD [MLM, 2015], a junior Australian exploration company. The deposit in question is SCONI's Greenvale deposit. SCONI is a mining project in development by Metallica Minerals, located $250 \mathrm{Km}$ west of Townsville (North Queensland). The project has been through the pre-feasibility stage, and is currently under funding stage. The company is seeking joint venture partners and funding for the construction of the High Pressure Acid Leaching (HPAL) processing plant, whose capital cost is estimated at approximately AU $\$ 1 \mathrm{Bi}$ [MLM, 2015].

Publicly-available data was used in this analysis, to reinforce the versatility of this valuation model. Both commodity price data, costing, and metallurgical data were obtained from previous studies performed on these topics. The resource estimation for the deposit is presented in Table 4.1 (adapted from Metallica's Greenvale in-situ data):

Mine valuation will consist on the following procedure:

- Determination of yearly throughputs and life of mine 
Table 4.1: Greenvale resource table Greenvale Deposit

\begin{tabular}{|l|l|l|l|l|}
\multicolumn{5}{|c|}{ Greenvale Deposit } \\
\hline Measured & Tonnes (Mt) & $\mathrm{Ni}(\%)$ & Co $(\%)$ & $\mathrm{Sc}(\mathrm{g} / \mathrm{t})$ \\
\hline Measured & 3.5 & 0.98 & 0.08 & 39 \\
\hline Indicated & 5.4 & 0.94 & 0.07 & 38 \\
\hline Inferred & 0.6 & 0.93 & 0.07 & 37 \\
\hline Total & 9.5 & 0.95 & 0.07 & 39 \\
\hline
\end{tabular}

- Selection of price forecast that resembles the economic scenario from 2012 to 2016

- Metallugical and financial data input

- Mine valuation algorithm run for standard, real option and dynamic option models

- Plotting data for visualization

- Comparison of results from the three methods

By assuming a 10-year life of mine, the following parameters were generated:

- Total reserve: $3.5 \mathrm{Mt}$ of ore at $0.98 \% \mathrm{Ni}, 0.08 \% \mathrm{Co}$ and $39 \mathrm{~g} / \mathrm{t} \mathrm{Sc}$

- Mine yearly throughput: 350,000 tonnes/annum

- Mill yearly throughput: 320,000 tonnes/annum

- Throughput modification allowed: $+-5 \%$ of the default capacity

- Buffer stockpile maximum capacity: 70,000 tonnes

- Sales rate: Maximum product tonnage available

The yearly throughput determination was based on estimates targeted by nickel laterite feasibility studies [Willis and Gifford, 2010, MLM, 2013], which included the use of by-products for diversification of revenue generation. 
For commodity pricing, a forecast was generated using the proposed scenario of decreasing trends both for nickel and cobalt. Using data from 1980 to 2012, a total of 11 years of future values were generated. Scandium contains a static price, given the lack of available historical data for manipulation of the commodity prices. Figure 4.7 shows the forecasted prices compared to the historical data [InvestentMine, 2016] from 2012 to 2015 . The method selected for forecasting was mean-reverting drift (HWV), with regular volatility (1 sigma) and a mean of $0.8 \mu$.

It is interesting to notice that the forecasted prices were able to capture the commodity trend and volatility for nickel. Cobalt's price volatility was also captured. The simulation proved to be succesful to generate forecasted prices. The shortcomings of not being able to forecast the exact historical price will be overcome with the generation of several price scenarios and multiple simulations inside each of them.

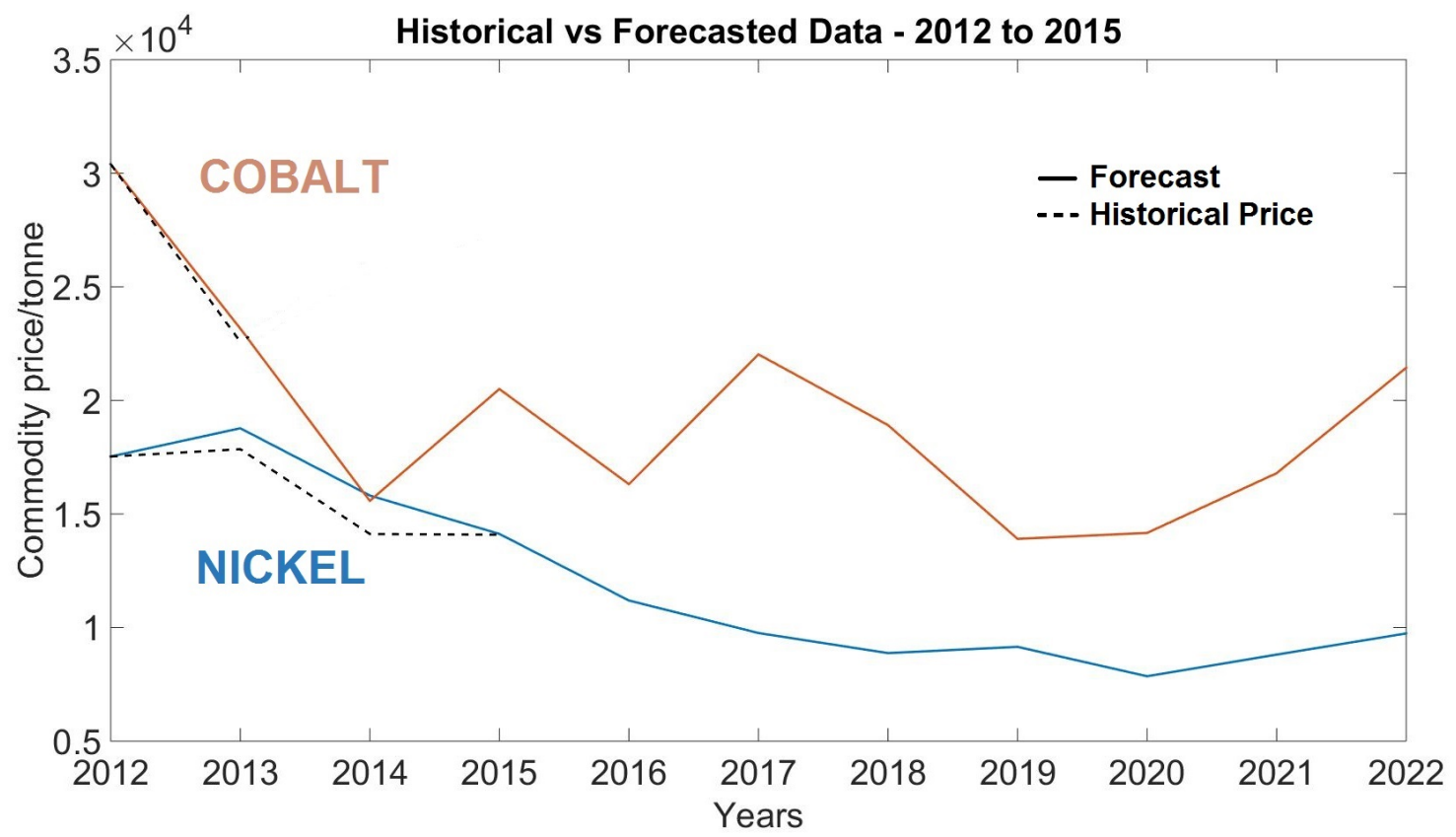

Figure 4.7: Commodity prices used on mine valuation

Financial and extractive metallurgy data was collected for nickel laterite deposits [Crundwell, 2011] with specific scandium metallurgical recovery data [Wang et al., 2011]. The study assumed an operation which would comprise both mining, processing and refining the material through an acid leaching plant, and the final product would consist on metallic nickel, metallic cobalt and scandium oxide $(99.99 \%$ pu- 
rity). The data regarding commodity metallurgical recovery, determination of final product and grade is in Table 4.2 .

Table 4.2: Material specification

\begin{tabular}{|c|c|c|c|}
\hline Commodity & Metallurgical Recovery & Final Product & Product purity \\
\hline Nickel & $92 \%$ & Metallic nickel & $99 \%$ \\
\hline Cobalt & $90 \%$ & Metallic cobalt & $99 \%$ \\
\hline Scandium & $85 \%$ & Scandium oxide & $99.99 \%$ \\
\hline
\end{tabular}

Finally, the financial data used for the proposed hypothetical operation iis presented in Table 4.3 [Crundwell, 2011]. For this study, a discount rate of $10 \%$ was selected to conduct the mine valuation.

Table 4.3: Capital and Operating Costs

\begin{tabular}{|l|l|l|}
\hline \multicolumn{1}{|c|}{ Cost parameter } & Value(US\$/tonne) & \multicolumn{1}{c|}{ Material } \\
\hline Capital & 60000 & Mean milled ore tonnage per year \\
\hline Mining & 1500 & Milled ore \\
\hline Processing/Refining & 8500 & Milled ore \\
\hline Pre-stockpiling & 0.50 & Stockpiled tonne \\
\hline Product stockpiling & 2.00 & Stockpiled tonne \\
\hline Sales & 5.00 & Sold tonne \\
\hline
\end{tabular}

\subsection{Results}

Figure 4.8 represents the behavior of the main parameters in discussion (mine and mill throughputs, stockpile inventory) for the period of 11 years. An extra year was added to simulation, to allow milling of remaining material contained on the stockpile inventory.

The standard valuation methodology used constant throughput values and held the maximum available stockpile inventory for most of the time. The real option valuation model targeted a more aggresive strategy, focusing on ramping up the production in the early operation years. It respected the maximum allowed limit of $105 \%$ capacity for the mine and mill, while decreasing throughput values in the later operation years, as prices dropped. The dynamic valuation model, on the other 


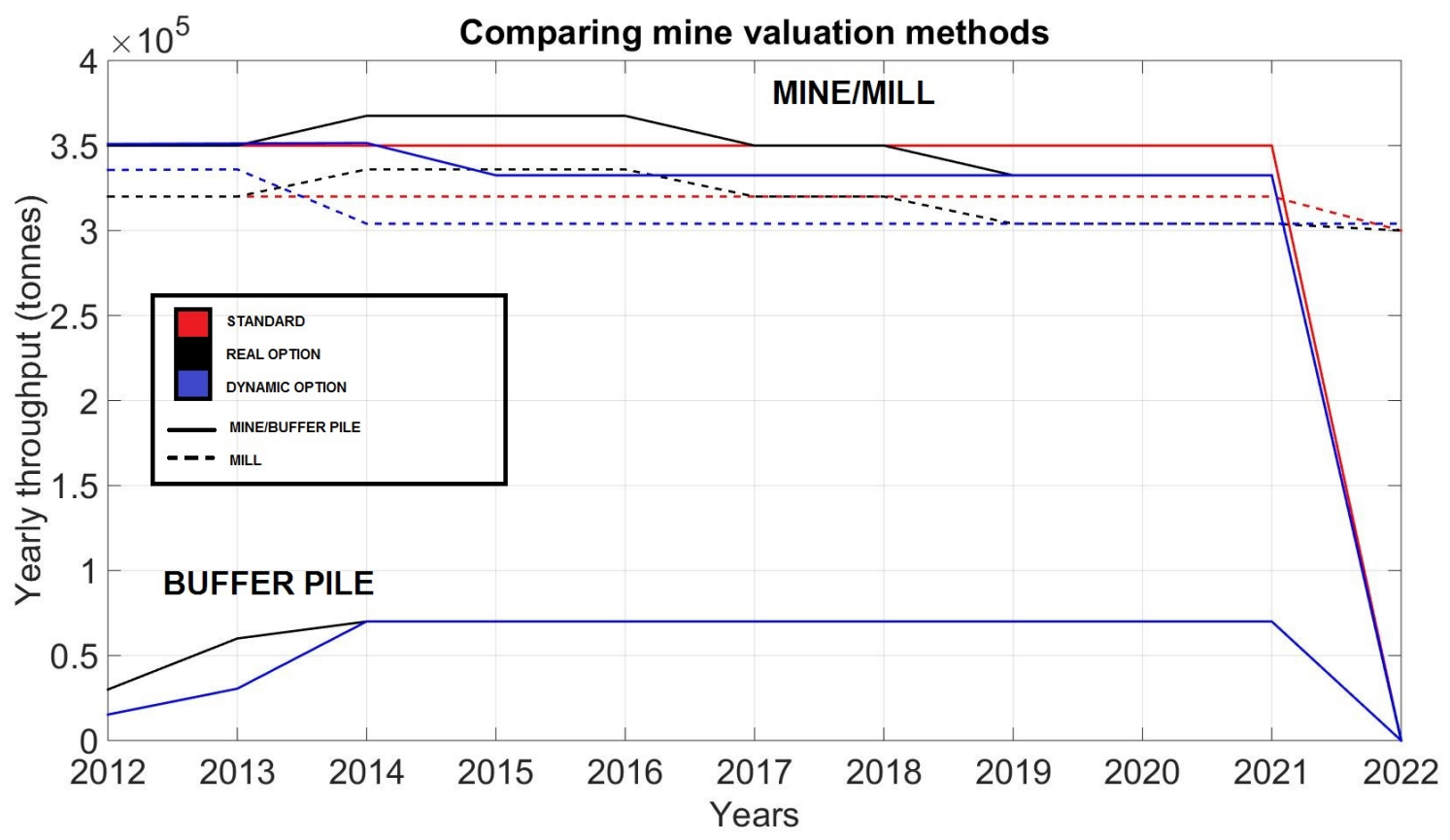

Figure 4.8: Mine Valuation Methods Comparison

hand, assumed a conservative posture. The mine started production with standard capacity, and, after year the third year, the production dropped to the minimum value allowed.

The mill, on the other hand, initiated production with the maximum allowed capacity and dropped after the third year to the minimum allowed capacity, in virtue of the decreasing commodity prices. The buffer pile, subsequently, took longer to build up the maximum allowed inventory, which then stabilized until the final year of operation.

The final NPV results are as follows:

Table 4.4: NPV results

\begin{tabular}{|c|c|c|c|}
\hline Method & NPV (US\$ Mi) & $\%$ Reserve Mined & \% Increase \\
\hline Standard & 128.01 & $100 \%$ & - \\
\hline Real Option Valuation & 130.90 & $100 \%$ & $2.26 \%$ \\
\hline Dynamic Option Valuation & 133.62 & $96.5 \%$ & $4.38 \%$ \\
\hline
\end{tabular}


These results demonstrate the efficacy of the dynamic valuaaion method over the existing alternatives. A $2.29 \%$ increase was observed by using the proposed model in comparison to the real option methodology. In fact, it is interesting to notice that the optimization method decided on not mining the deposit entirely, most certainly because commodity prices were not favorable.

\subsection{Summary and Conclusions}

Mineral asset valuation was performed for a hypothetical Ni-Co-Sc operation consisting of a mine, processing plant and leaching plant. Three different valuation merhodologies were investigated: Standard discounted cash-flow analysis, real option valuation and dynamic option valuation method.

A price forecasting methodology using mean-reverting drift was applied to obtain future prices for nickel and cobalt for a period of 11 years. Combining this data with financial and extractive metallurgy data of nickel laterite deposits, optimal throughput parameters for the proposed operation were determined.

The study identified that both the real option valuation and the dynamic model obtained NPV values that exceeded the standard cash flow model. However, the ROV model allowed a $2.26 \%$ increase in NPV, while the DOV presented $4.38 \%$ increase. The new proposed methodology and its stochastic emphasis proved to be successful for the determination of optimal operating parameters, showing the qualities of operational flexibility for mine valuation. 


\section{Chapter 5}

\section{Optimization Methods}

Optimization is an inherent part of engineering, normally used in association with mathematical models to obtain process parameters or work conditions. In a classical definition, an optimization problem corresponds to maximizing or minimizing a previously built mathematical function, modifying the input parameters values in order to obtain an optimal performance point. In the case of the economic models previously specified in this thesis, the ultimate objective is to obtain the highest Net Present Value (NPV) for the hypothetical mining project in question.

The methods presented in this chapter were also used on the dynamic valuation performed in the previous chapter, and will be thoroughly described here. The use of computer algorithms for optimization required the selection of a platform that would allow data storage, numerical methods for optimization, statistical analysis and data plottting. MATLAB constains a group of function for optimal parameter identification satisfying contraints, including solvers for linear programming, mixedinteger linear programming, quadratic programming, nonlinear optimization, and nonlinear least squares [Mathworks, 2016a]. For the purpose of this project, the toolbox includes two optimization methods that will be described in thie thesis: Genetic Algorithm and Interior Point Method.

\subsection{Genetic Algorithm}

The genetic algorithm contained in Matlab presents a set of functions are part of the Global Optimization Toolbox, developed by Houck [Houck et al., 1995]. It contains modules presented as MATLAB functions, distributed in several ".m" files, 
which allow specific calls to any function and also allows flexibility of altering the function parameters according to the objective or simulation calibration. The package provides all the main functions that are used in a standard genetic algorithm, containing:

- Random generator of initial population

- Gene selection with binary or real representation

- Configuration of mutation and crossover parameters

- Selection of mutation and crossover parameters

- Individual selection process

- Selection of mutation end parameters

The formulation of the genetic algorithm optimization procedure is as follows:

$\min \mathrm{f}(\mathrm{x})$

subject to

$\mathrm{c}(\mathrm{x}) \leq 0$

$\operatorname{ceq}(x)=0$

$A \cdot x \leq b$

Aeq $\cdot x=b e q$

$l b \leq x \leq u b$

where

- $x$ is a single row vector containing the input variables

- $f(x)$ represents the function to be minimized 
- $c(x)$ is a function that represents non-linear inequality constraints

- $\operatorname{ceq}(x)$ is a function that represents non-linear equality constraints

- $A$ is the matrix used to represent linear inequality constraints

- $b$ is a vector used to represent linear inequality constraints

- $A_{e q}$ is a matrix used to represent linear equality constraints

- $b_{e q}$ is a vector usd to represent linear equality constraints

- $l b$ is a vector used to represent the lower boundary for $\mathrm{x}$ values

- $u b$ is a vector used to represent theupper boundary for $\mathrm{x}$ values

The standard syntax for the genetic algorithm is as follows:

$$
[x, f v a l]=g a(\text { functionname }, N v a r, A, b, \text { Aeq }, \text { beq }, l b, u b)
$$

where

- $x$ corresponds to a vector containing the optimized value for each of the problem variables

- $f v a l$ is the optimized function output

- @ functionname is the declaration of tge funtion to be minimized

- Nvar corresponds to the number of variables to be optimized

- $A, b, A e q$ and beq are the same as the fmincon formulation depicts

- $l b$ and $u b$ correspond to lower and upper boundary limits, respectively 


\subsection{Interior Point Method}

MATLAB employs the Interior Point Method method through a routine called Fmincon. It is a non-linear solver contained in the Optimization Toolbox, which can be accessed and used both from the Toolbox or through the generation of coded script. It relies on linear programming methods, and allows the use of continuous variables and defined constraints by the user. Compared to the genetic algorithm, Fmincon finds local minimums of functions.

These functions must be developed and saved using MATLAB structure, and require certain rules to be valid. The functions, however, may possess non-linear behavior in its structure. The most important rule while setting up optimization using Fmincon is the mandatory use of a row vector as the input for the main function to be optimized, otherwise the function will not be accepted by the system. The formulation for the optimization is as follows:

$\min \mathrm{f}(\mathrm{x})$

subject to

$\mathrm{c}(\mathrm{x}) \leq 0$

$\operatorname{ceq}(x)=0$

$A \cdot x \leq b$

Aeq $\cdot x=b e q$

$l b \leq x \leq u b$

where

- $x$ is a single row vector containing the input variables 
- $f(x)$ represents the function to be minimized

- $c(x)$ is a function that represents non-linear inequality constraints

- $c e q(x)$ is a function that represents non-linear equality constraints

- $A$ is the matrix used to represent linear inequality constraints

- $b$ is a vector used to represent linear inequality constraints

- $A_{e q}$ is a matrix used to represent linear equality constraints

- $b_{e q}$ is a vector usd to represent linear equality constraints

- $l b$ is a vector used to represent the lower boundary for $\mathrm{x}$ values

- $u b$ is a vector used to represent theupper boundary for $\mathrm{x}$ values

The standard syntax for Fmincon is as follows:

$$
[x, f v a l]=f \operatorname{mincon}(\text { functionname }, x 0, A, b, A e q, b e q, l b, u b)
$$

where

- $x$ corresponds to a vector containing the optimized value for each of the problem variables

- fval is the optimized function output

- @ functionname is the declaration of the funtion to be minimized

- $x 0$ corresponds to the starting point of values to be evaluated by the solver

- $A, b, A e q$ and $b e q$ are the same as the fmincon formulation depicts

- $l b$ and $u b$ correspond to lower and upper boundary limits, respectively 


\subsubsection{Evaluation of solvers for DOV}

At first, both optimization methods were used for the DOV calculation. The use of the genetic algorithm proved to be a challenge in regard to the amount of time required to calculate each iteration of the model.

The optimization plan was modified and incorporated a more time-efficient optimization algorithm denominated Fmincon. In addition, a cross-validation of selected results was performed using the genetic algorithm. Early studies demonstrated that the genetic algorithm required between 28 minutes to one hour to complete one iteration of the mine valuation model. Fmincon, on the other hand, provided virtually the same results requiring approximately 20 to 30 seconds of algorithm running time.

Figure 5.1 shows the analysis of variance of Fmincon results compared to the genetic algorithm:

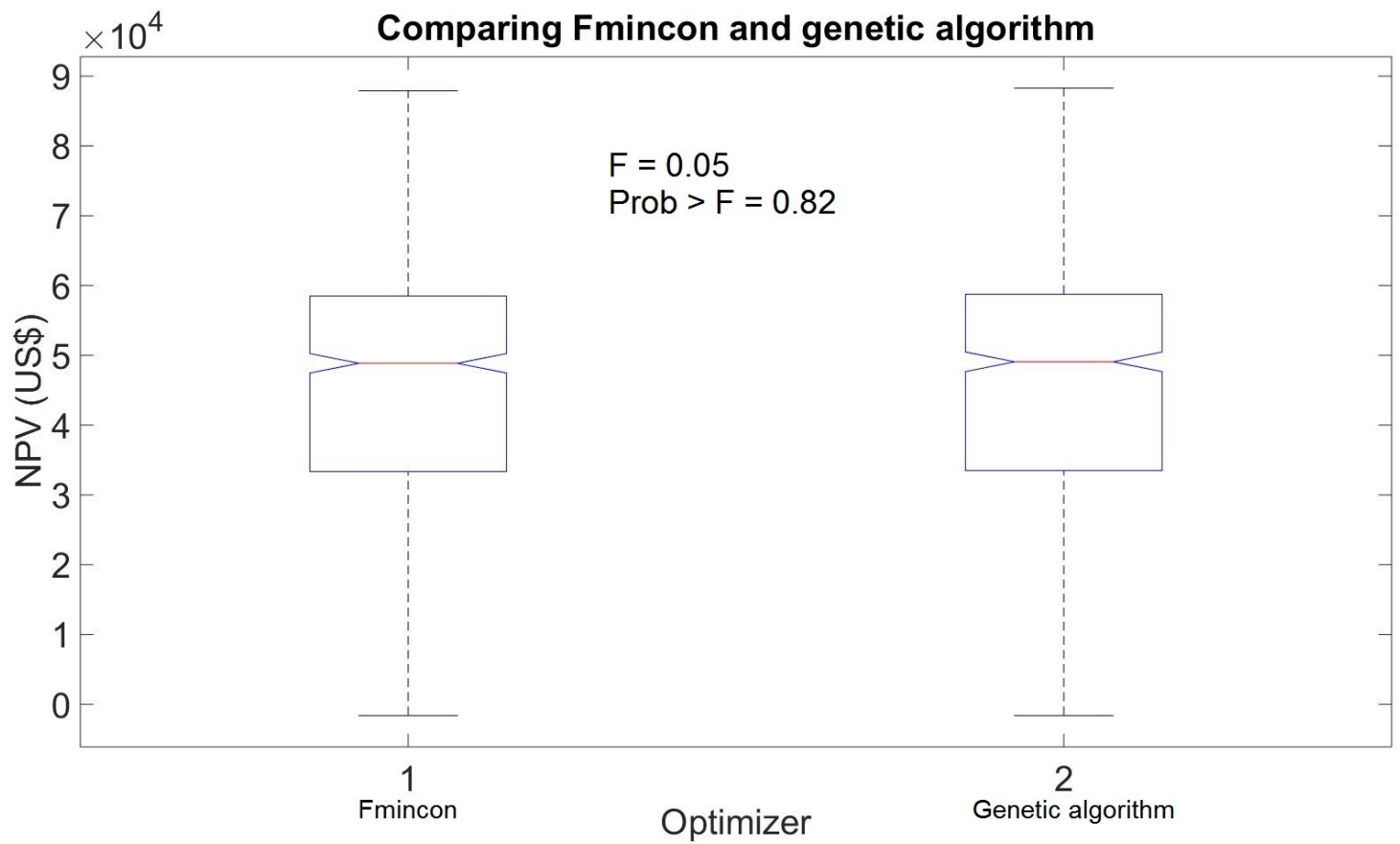

Figure 5.1: Fmincon approximation to the genetic algorithm

The analysis shows an $\mathrm{F}$ ratio of 0.05 with the $\mathrm{p}$-value $=0.82$. This very high probability of equal means allows the conclusion that the non-linear solver method- 
ology proved its capabalities of generating optimization for the overall experiment design scenarios proposed in this thesis. As for the genetic algorithm, it was used to cross-validate a sample of iterations, as well as double-check values obtained in cases where the values were inadequate or suspicious.

\subsubsection{Determining the required number of function evalua- tions}

The Fmincon solver is a complex algorithm which runs under the MATLAB interface. It relies on a search engine that evaluates a pre-established MATLAB function and determines the minimum value by going through the middle of the solid defined by the problem, instead of its surface [Forsgren et al., 2002]. One of the main parameters used by the solver is the number of function evaluations it is allowed to use before achieving its objective.

A study on the required number of function evaluations was performed, in order to reduce running time for the algorithm. It was observed that the algorithm would reach an average of 8,000 to 12,000 evaluations to obtain the optimal function value. with increasing time demand with higher number of function evaluations. The same algorithm was evaluated with distinct maximum number of function evaluations, and the results are presented below in Figure 5.2.

The default number of function evaluations (3000) was sufficient to achieve an optimized value corresponding to $99.75 \%$ of the overall function minimum, using a total of 1 hour for completion. Subsequently, a higher value of function evaluations was proposed (4000), allowing the optimizer to obtain a value of $99.99 \%$ close to the minimum.

The decision consequently was the selection of a balance between running time and performance. A number of 4000 function evaluations was seleced to run the proposed experiments described in the research. 


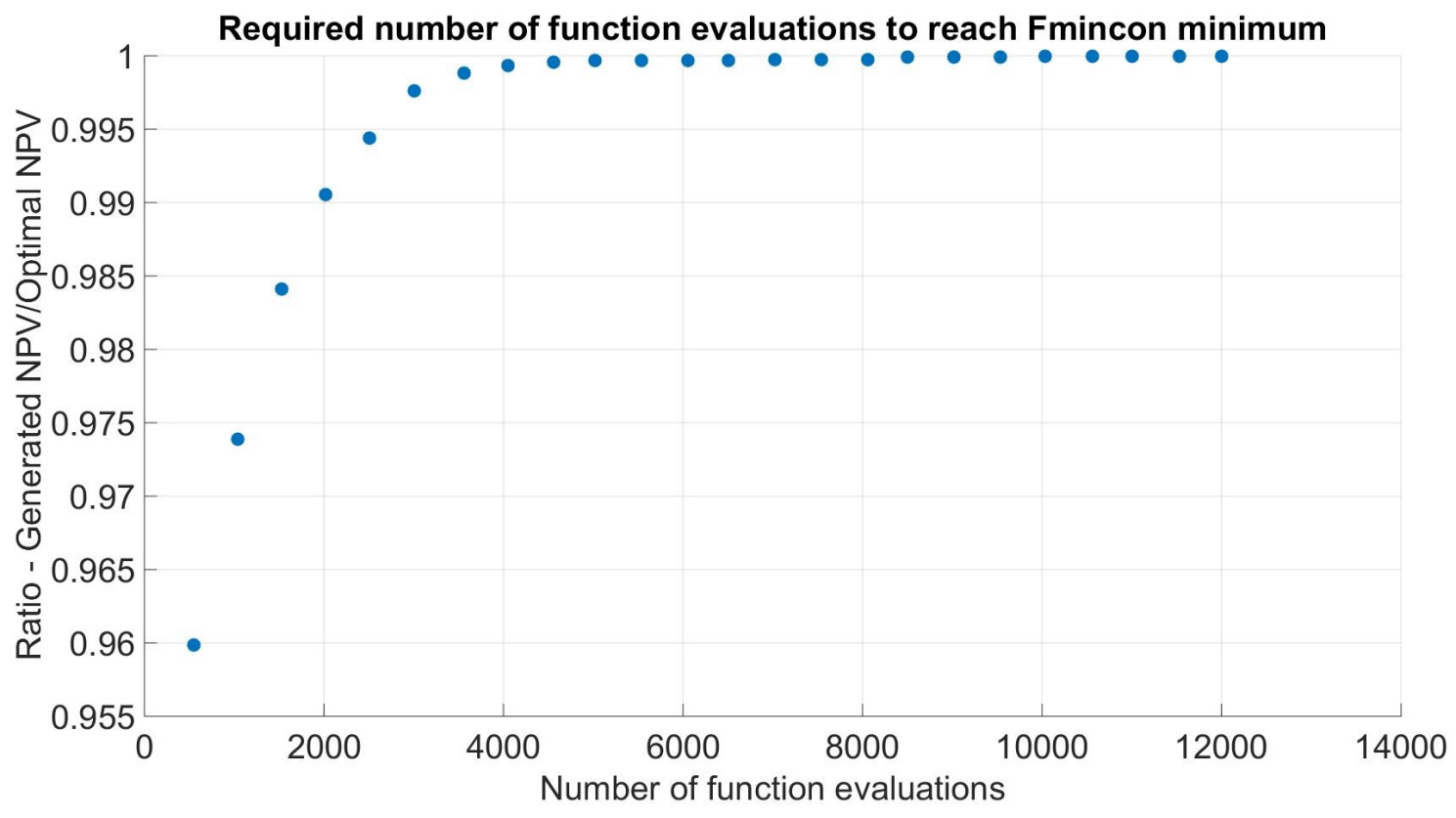

Figure 5.2: Required number of function evaluations to reach Fmincon minimum

\subsection{DOV Problem Formulation}

\subsubsection{Dynamic modeling procedure}

A minimization objective function was generated for the algorithm. It relies on the input of a single column vector denominated $\mathrm{x}$, which contains the following inputs: 


$$
\left[\begin{array}{c}
m t_{1} \\
\ldots \\
m t_{n} \\
m i_{1} \\
\ldots \\
m i_{n} \\
c 1_{1} \\
\ldots \\
c 1_{n} \\
c 2_{1} \\
\ldots \\
c 2_{n} \\
c 3_{1} \\
\ldots \\
c 3_{n}
\end{array}\right]=\left[\begin{array}{c}
x_{1} \\
\ldots \\
x_{n} \\
x_{n+1} \\
\ldots \\
x_{2 n} \\
x_{2 n+1} \\
\ldots \\
x_{3 n} \\
x_{3 n+1} \\
\ldots \\
x_{4 n} \\
x_{4 n+1} \\
\cdots \\
x_{5 n}
\end{array}\right]
$$

where:

- $\mathrm{n}$ corresponds to the year of operation (1 to 11 , in the context of the project)

- $\mathrm{x}$ corresponds to the vector position, ranging from 1 to $(5 \cdot n)$

- $m t_{n}$ corresponds to the mine throughput in year $\mathrm{n}$

- $m i_{n}$ corresponds to the mill throughput in year $\mathrm{n}$

- c1 corresponds to the sales rate of commodity 1 (Nickel) in year $n$

- $\mathrm{c} 2$ corresponds to the sales rate of commodity 2 (Cobalt) in year $\mathrm{n}$

- c3 corresponds to the sales rate of commodity 3 (Scandium) in year $n$

This syntax in particular is specific for an operation with the three commodities in study, but it could be modified for a single commodity or more than three products, depending on the objective. The final matrix for evaluation encompasses all the throughput and sales values specified above, containing a total of 55 rows (based on 11 years of operation).

The first constraint specified corresponds to the linear inequalities of the project. In this example, the total material mined and processed must be less than the total 
mine reserve. In the same fashion, the total sales of each commodity must be less than the the total product generated in the operation. The following list specifies the linear inequalities developed:

- Total material mined is less than or equal to mine reserve (b1)

- Total material processed is less than or equal to mine reserve $(b 2)$

- Total nickel sold is less than or equal to total nickel produced (b3)

- Total cobalt sold is less than or equal to total cobalt produced (b4)

- Total scandium sold is less than or equal to total scandium produced (b5)

The formulation constitutes itself on a matrix multiplication, using a matrix similar to an identity one. The visual representation for $A \cdot x \leq b$ goes as follows:

$$
\left[\begin{array}{l}
11 \ldots 1100 \ldots 0000 \ldots 0000 \ldots 0000 \ldots 00 \\
00 \ldots 0011 \ldots 1100 \ldots 0000 \ldots 0000 \ldots 00 \\
00 \ldots 0000 \ldots 0011 \ldots 1100 \ldots 0000 \ldots 00 \\
00 \ldots 0000 \ldots 0000 \ldots 0011 \ldots 1100 \ldots 00 \\
00 \ldots 0000 \ldots 0000 \ldots 0000 \ldots 0011 \ldots 11
\end{array}\right] \cdot\left[\begin{array}{c}
x_{1} \\
x_{2} \\
x_{3} \\
\ldots \\
x_{i}
\end{array}\right] \leqslant\left[\begin{array}{c}
b_{1} \\
b_{2} \\
b_{3} \\
b_{4} \\
b_{5}
\end{array}\right]
$$

where:

- A corresponds to a multiplication matrix

- $\mathrm{x}$ corresponds to the input vector

- $\mathrm{b}$ corresponds to the linear inequality maximum values, with the sequence mine, mill, and sales (Ni-Co-Sc)

- $\mathrm{i}$ corresponds to the vector position in $\mathrm{x}$

Finally, the lower and upper boundary constraints are declared on the algorithm. They correspond to minimum and maximum values that a certain input parameter may possess during the operation. The algorithm search an optimal value between these boundaries and outputs it as a result. The matrix syntax goes as follows: 


$$
\left(1-\mathrm{M}_{f}\right) \cdot\left[\begin{array}{c}
x_{1} \\
x_{2} \\
x_{3} \\
\ldots \\
x_{i}
\end{array}\right] \leqslant\left[\begin{array}{c}
x_{1} \\
x_{2} \\
x_{3} \\
\ldots \\
x_{i}
\end{array}\right] \leqslant\left[\begin{array}{c}
x_{1} \\
x_{2} \\
x_{3} \\
\ldots \\
x_{i}
\end{array}\right] \cdot\left(1+M_{f}\right)
$$

$M_{f}$ corresponds to a modifying factor that widens the possible outcomes of the input parameters. In general terms, it declares the percentage of change allowed in the throughput and sales of commodities. Using a factor of 0.2 would allow the mine and mill to operate between $+-20 \%$ of their default capacity. In the same fashion, the sales could be increased in up to $20 \%$, or even decreased in case the optimizer detects this movement will benefit revenue generation.

Nevertheless, allowing the mine and mill to work under uncertainty required the use of a dynamic buffer pile. It will serve as an inventory for material to be deposited and withdrawn from, with no limitation on maximum production by the mill if the mine has not produced enough material. The visual representation of this methodology is in Figure 5.3.

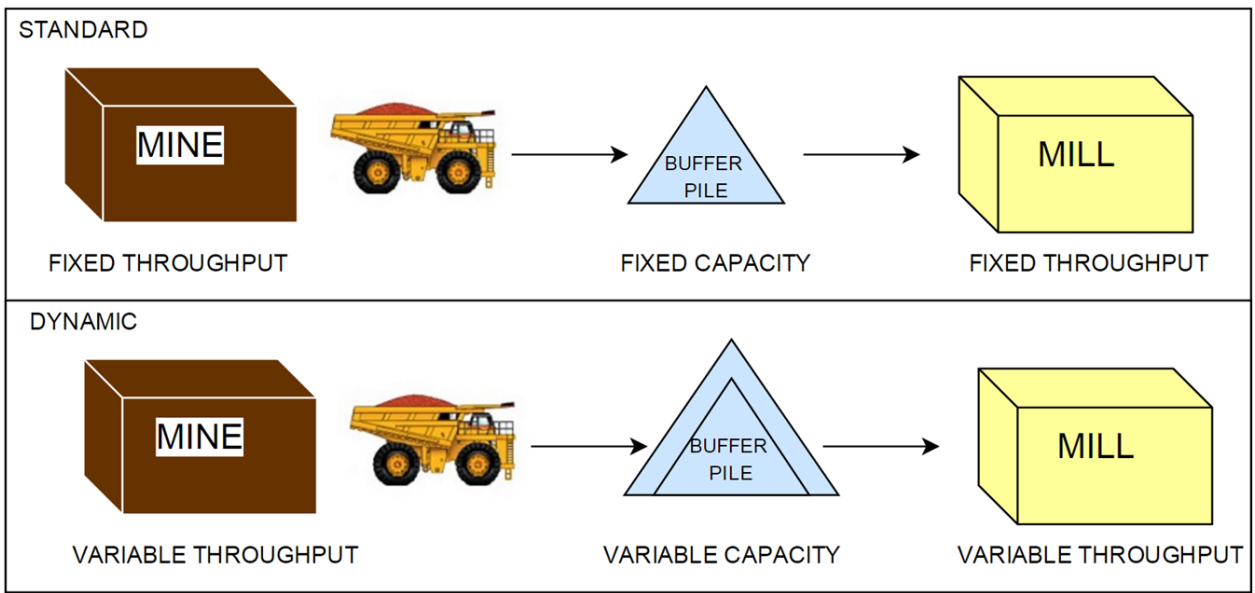

Figure 5.3: Interface between mine and mill in the dynamic model

\subsubsection{Generating a complex system}

The necessity to run the optimization algorithm for several times, including testing, experimental procedures and validation, required the creation of a system capable of 
procedure automation. The objective was to generate a single algorithm that would load price forecasts, run the mine valuation model and the optimization procedure for all the scenarios presented in the experiment design automatically.

The solution was the generation a system that combines simulation and optimization, developing loops on stochastic processes that ultimately generate the requested resuls. An overview of the system is presented in Figure 5.4:

COMPLEX SYSTEM FOR MINE VALUATION

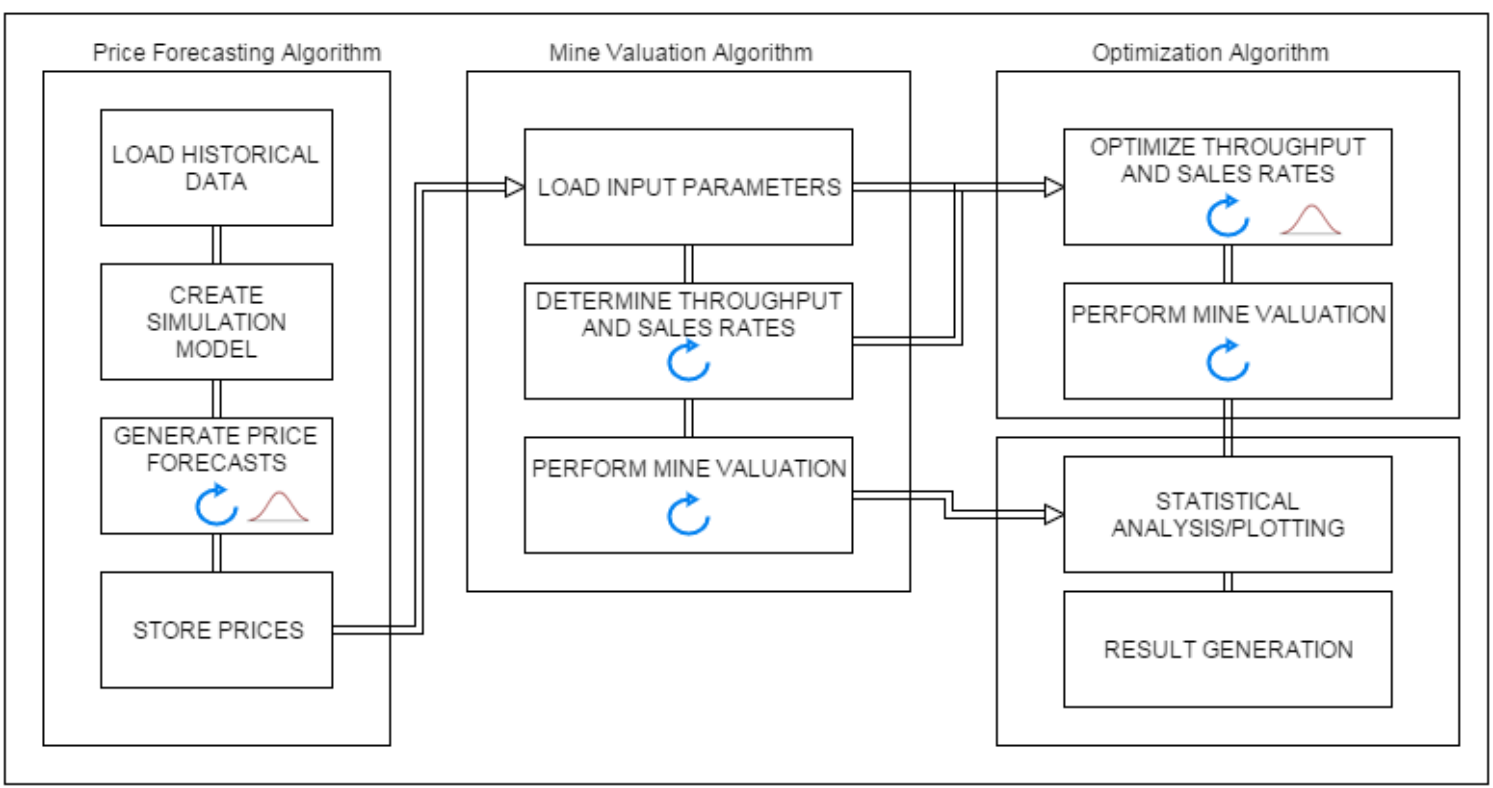

Figure 5.4: Complex System for Mine Valuation

The picture represents the complex system developed for this project. The cycle arrow depicts simulation (iteration process), while the distribution figure depicts stochastic processes taking place.

\subsection{Summary and Conclusions}

A complex system for mine valuation was created, developed entirely in MATLAB and taking advantage of its combination of data management, optimization and simulation capabilities. A hypothetical Ni-Co-Sc mineral project was evaluated through 
this system.

The proposed methodology comprises two types of optimization techniques (genetic algorithm and interior point method) to establish mineral asset valuation using forecasted nickel and cobalt prices. These prices were generated through stochastic differential equations models and created for several different commodity market scenarios. 


\section{Chapter 6}

\section{Results and Discussion}

In order to assess the utility of the DOV methodology in a realistic setting, two case studies were conducted. The first corresponds to a pilot scale valuation for Greenvale nickel project by Metallica Minerals, assuming the mining of 1,000 tonnes of nickel ore, with a default capacity of 100 mined tones per year. The second analysis was the mineral valuation of the Greenvale nickel deposit using industrial production rates, and targetting the deposit mining until full exhaustion. For this part, industrial throughput values were researched and selected [MLM, 2013, Crundwell, 2011].

For each of the proposed case studies, four different cases were evaluated:

- CASE 1: Constrained Buffer Pile, 10\% Discount Rate

- CASE 2: Regular Buffer Pile, 10\% Discount Rate

- CASE 3: Constrained Buffer Pile, 15\% Discount Rate

- CASE 4: Regular Buffer Pile, 15\% Discount Rate

Constrained piles refer to a mine valuation design in which the buffer pile can hold a limited amount of material. This difference in pile size was used to evaluate the impact of maximum allowed inventory in mine valuation. The subsequent sections will explain the specific parameters used and procedures taken on different scenarios.

In addition to DOV, standard DCF valuations were also conducted as a baseline in this study. 


\subsection{Base study - Pilot scale valuation}

The pilot-scale study was based on the initial developments done with the dynamic model. This initial analysis intended to evaluate the optimizers for calibration, therefore the selected operating parameters were lower in this stage. Using a target throughput of 100 mined tonnes per year, the optimization procedure could be performed faster, compared to using values in industrial scale. The search algorithm was able to find optimum values faster in a smaller range of search. The technical data for the study corresponds to the following:

- Total reserve: 1000 tones of ore at $0.98 \% \mathrm{Ni}, 0.08 \% \mathrm{Co}$ and $39 \mathrm{~g} / \mathrm{t} \mathrm{Sc}$

- Mine yearly throughput: 100 tonnes/annum

- Mill yearly throughput: 95 tonnes/annum

- Throughput modification allowed: $+-20 \%$ of the default capacity

- Buffer stockpile maximum capacity: 20 tonnes (Constrained) and 100 tonnes (unlimited, and also referred as Regular throughout this document)

- Sales rate: At least $95 \%$ of the produced capacity

The financial and metallurgical data for this valuation procedure is the same as presented in chapter 4, with CAPEX scaled with production. NPV results results were compared between DCF and DOV, with values represented to evaluate the efficacy of value increase using the dynamic methodology. Table 6.1 represents the financial results for the four cases, in each scenario of the experiment design presented in chapter 3 .

Analyzing the data, the constrained pile scenarios achieved higher NPV values for all the scenarios with $10 \%$ discount rate. The regular pile methodology was only more valuable using a higher discount rate, and taking in consideration high price trends for nickel, as it can be visualized in scenarios 3, 4, 7, 8, 11, 15 and 16 (Cases 3 and 4$)$.

For cases with $10 \%$ dicount rate the DOV showed a $4 \%$ average increase over DCF. For Cases 3 and 4, however, the gain corresponded to approximately $11 \%$ on average. 
Table 6.1: Base study - Result data

\begin{tabular}{|c|c|c|c|c|c|c|c|c|}
\hline \multirow{2}{*}{ Scenario } & \multicolumn{2}{|c|}{ CASE 1 } & \multicolumn{2}{c|}{ CASE 2 } & \multicolumn{2}{c|}{ CASE 3 } & \multicolumn{2}{c|}{ CASE 4 } \\
\cline { 2 - 9 } & $\begin{array}{c}\text { NPV } \\
\text { (US\$ } \\
\text { K) }\end{array}$ & $\begin{array}{c}\text { Increase } \\
\text { over } \\
\text { DCF } \\
(\%)\end{array}$ & $\begin{array}{c}\text { NPV } \\
(\text { US\$ } \\
\text { K) }\end{array}$ & $\begin{array}{c}\text { Increase } \\
\text { over } \\
\text { DCF } \\
(\%)\end{array}$ & $\begin{array}{c}\text { NPV } \\
\left(\begin{array}{l}\text { US } \$ \\
\text { K) }\end{array}\right.\end{array}$ & $\begin{array}{c}\text { Increase } \\
\text { over } \\
\text { DCF } \\
(\%)\end{array}$ & $\begin{array}{c}\text { NPV } \\
\text { (US\$ } \\
\text { K) }\end{array}$ & $\begin{array}{c}\text { Increase } \\
\text { over } \\
\text { DCF } \\
(\%)\end{array}$ \\
\hline 1 & 45.42 & $7.92 \%$ & 45.14 & $6.23 \%$ & 30.30 & $17.27 \%$ & 29.94 & $16.10 \%$ \\
\hline 2 & 51.93 & $5.70 \%$ & 51.41 & $3.71 \%$ & 35.04 & $12.44 \%$ & 34.94 & $12.09 \%$ \\
\hline 3 & 61.34 & $3.97 \%$ & 60.58 & $1.94 \%$ & 41.17 & $7.06 \%$ & 41.57 & $8.11 \%$ \\
\hline 4 & 66.50 & $3.35 \%$ & 65.80 & $1.58 \%$ & 44.95 & $5.70 \%$ & 45.24 & $6.34 \%$ \\
\hline 5 & 45.21 & $8.31 \%$ & 44.86 & $6.45 \%$ & 30.01 & $16.22 \%$ & 29.73 & $15.59 \%$ \\
\hline 6 & 48.79 & $7.31 \%$ & 48.40 & $5.47 \%$ & 32.59 & $15.56 \%$ & 32.44 & $14.82 \%$ \\
\hline 7 & 60.16 & $4.57 \%$ & 59.53 & $2.72 \%$ & 40.62 & $8.61 \%$ & 40.73 & $8.87 \%$ \\
\hline 8 & 65.43 & $3.47 \%$ & 64.84 & $1.88 \%$ & 44.43 & $6.30 \%$ & 44.67 & $6.93 \%$ \\
\hline 9 & 18.75 & $10.05 \%$ & 18.66 & $7.16 \%$ & 6.64 & $18.35 \%$ & 6.24 & $16.89 \%$ \\
\hline 10 & 26.26 & $6.11 \%$ & 26.05 & $3.60 \%$ & 12.65 & $15.82 \%$ & 12.28 & $15.70 \%$ \\
\hline 11 & 52.28 & $2.80 \%$ & 51.60 & $0.65 \%$ & 32.52 & $6.33 \%$ & 32.63 & $6.50 \%$ \\
\hline 12 & 60.26 & $2.52 \%$ & 59.62 & $0.67 \%$ & 38.60 & $4.71 \%$ & 38.86 & $5.32 \%$ \\
\hline 13 & 20.75 & $8.30 \%$ & 20.70 & $5.82 \%$ & 8.13 & $14.76 \%$ & 7.65 & $15.62 \%$ \\
\hline 14 & 25.42 & $7.30 \%$ & 25.27 & $4.64 \%$ & 12.00 & $17.11 \%$ & 11.65 & $16.73 \%$ \\
\hline 15 & 53.03 & $2.97 \%$ & 52.43 & $0.88 \%$ & 33.24 & $6.73 \%$ & 33.27 & $6.54 \%$ \\
\hline 16 & 59.62 & $3.06 \%$ & 58.90 & $1.10 \%$ & 38.38 & $5.57 \%$ & 38.53 & $5.83 \%$ \\
\hline Average & - & $5.48 \%$ & - & $3.41 \%$ & - & $11.16 \%$ & - & $11.12 \%$ \\
\hline
\end{tabular}

Standard deviations corresponded to 2.5\% (Cases 1 and 2) and 4.8\% (Cases 3 and $4)$.

The following plots (Figures 6.1 to 6.6) show the three parameters subjected to uncertainty while optimization was being performed: Mine throughput, mill throughput and product sales. Each of the by-products will be shown in plots. A total of 800 simulations were combined for the generation of these plots (all the 16 scenarios in the experiment design, with 50 simulations in each). In each of the plots, errorbars represent mean and standard deviation of the simulated values.

For the mine throughput, all the cases targeted a strategy of higher productiion during the initial years. Subsequently, production achieved its minimum value and continued until depletion of the deposit. The only exception is for Case 2, in which 

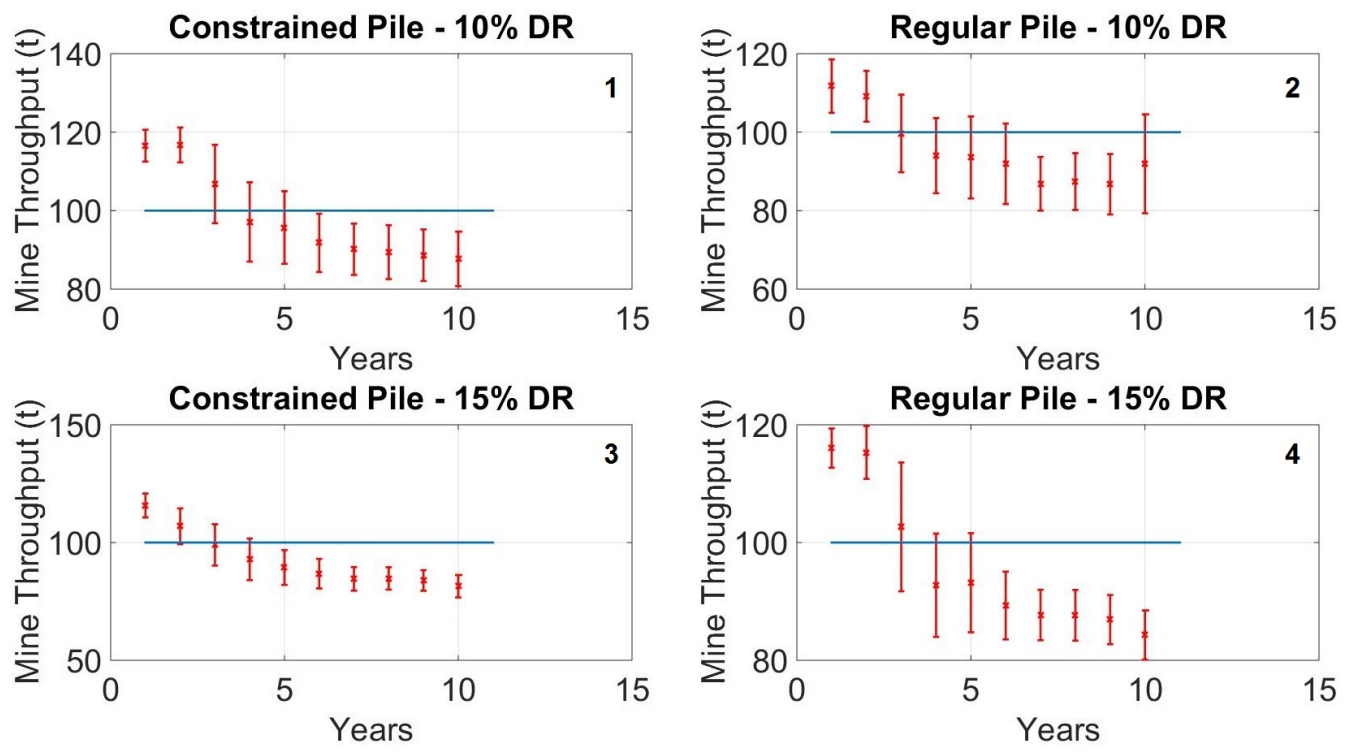

Figure 6.1: Mine throughput plotting - Base study

mine throughput increased in the final operating years.
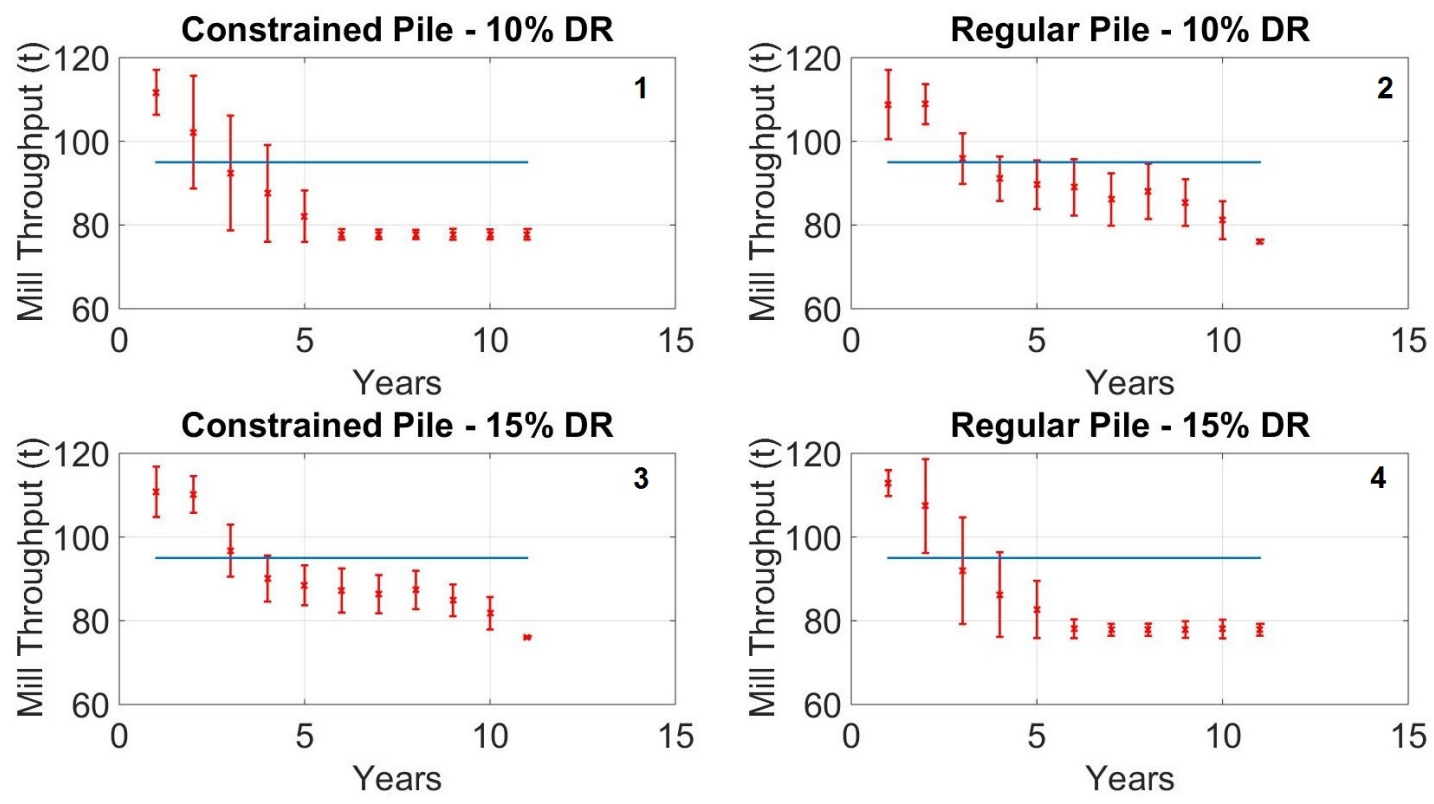

Figure 6.2: Mill throughput plotting - Base study 
The model demonstrated that the optimal scenario for the mill would be working over its rated capacity udring the initial years, and gently decresing in capacity over the years. This modification is more subtle in Cases 2 and 3, while the others have a more accentuated drop during medium term (years 2 to 5 ).
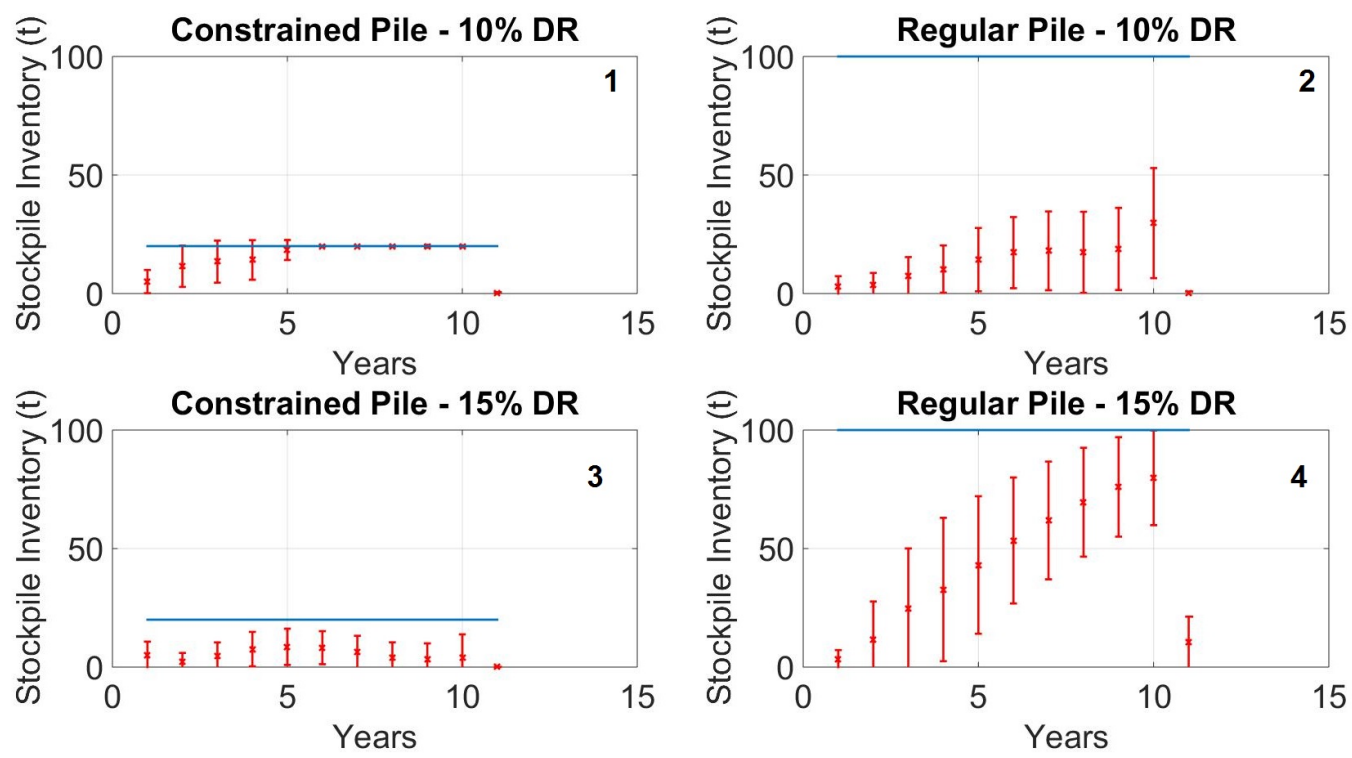

Figure 6.3: Buffer pile inventory plotting - Base study

Buffer pile inventory in this study was substiantially affected by the parameters in each case. Case 1 specifies a trend with growing inventory until it reaches maximum capacity by year 5. Then, it is kept constant until the end operation. The same behavior is observed in Case 4, with maximum capacity achieved by the end of year 10 .

For case 2, the inventory was increased along time, however, it never surpassed half of the maximum allowed capacity. Finally, case 3 exemplifies the priciple of varying buffer capacity along the years. The pile has shifting mean values and keeps its inventory below maximum capacity at all times.

Product sales were consistent with the mill/smelter production over the years. Even though the optimizer was allowed to selectively delay a small percentage of commodity sales, such practive was not used with intensity. Therefore, the tonnage produced by the mill is very consistent with the amouunt of material produced each year. 

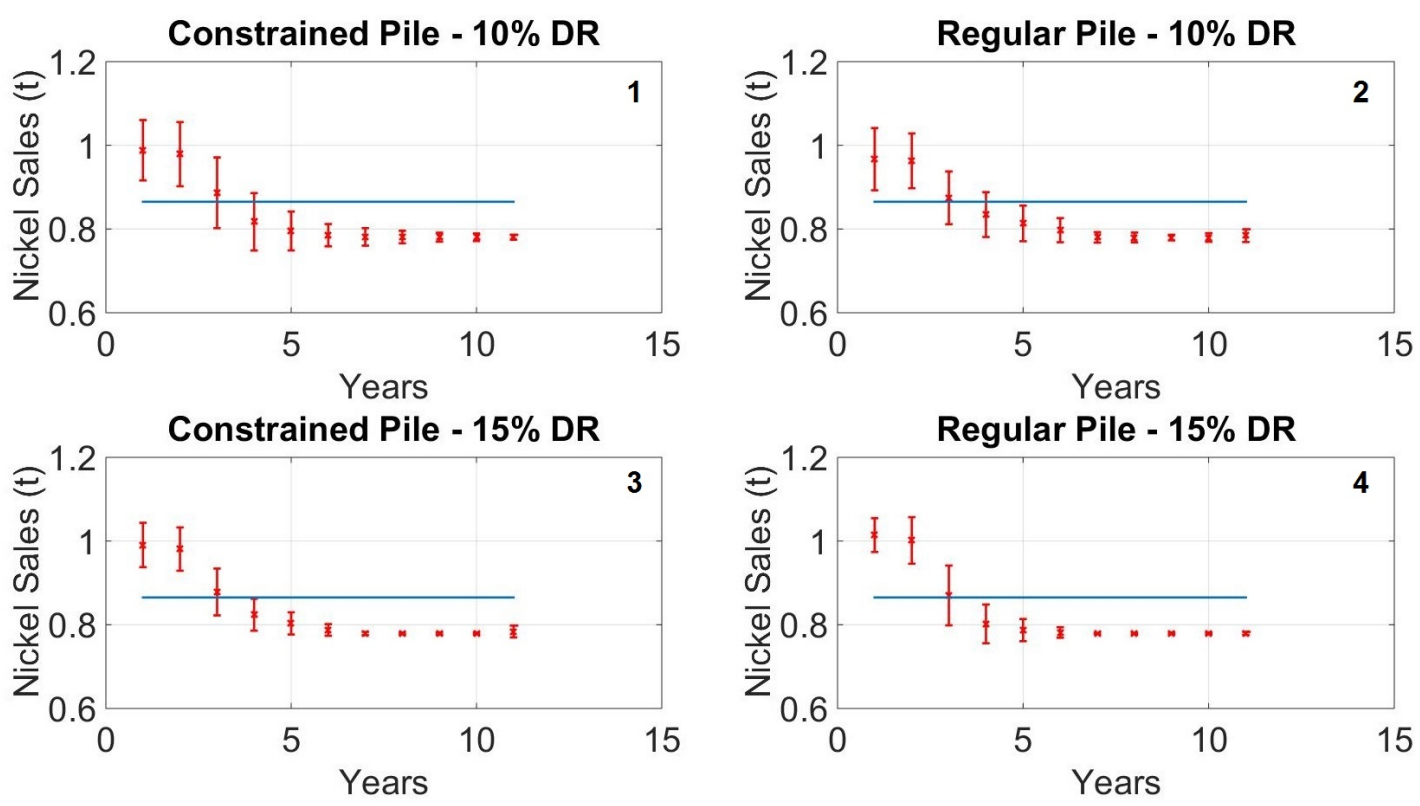

Figure 6.4: Nickel sales plotting - Base study

Analyzing cases individually, it is noticeable that nickel and cobalt sales were constant throughout case analysis. The only noticeable difference is scandium sales, which remain highly variable for case 1 and not for the rest.

Finally, an analysis of the mean revenue per year was performed. A scatterplot of this parameter in comparison with the NPV Ratio (dynamic/standard) is presented in Figure 6.7, and allows some conclusions to be generated. First, for Cases 1 and 2, the first one is able to have higher NPV ratios values with the same mean revenue per year, which attests scenarios with constrained stockpile being more favorable than scenarios with unlimited stockpiling.

Case 3 is consistent in achieving high NPV ratios and for smaller values of mean revenue (an indication that commodity prices are going down, since all of them have the same starting point) the optimization generates maximum NPV percentage increase.

Case 4 is inconsistent in obtaining higher ratios for smaller revenue values. The distribution of points is the most scattered of all, and allows the interpretation that there are better alternatives to having an unlimited buffer pile. 

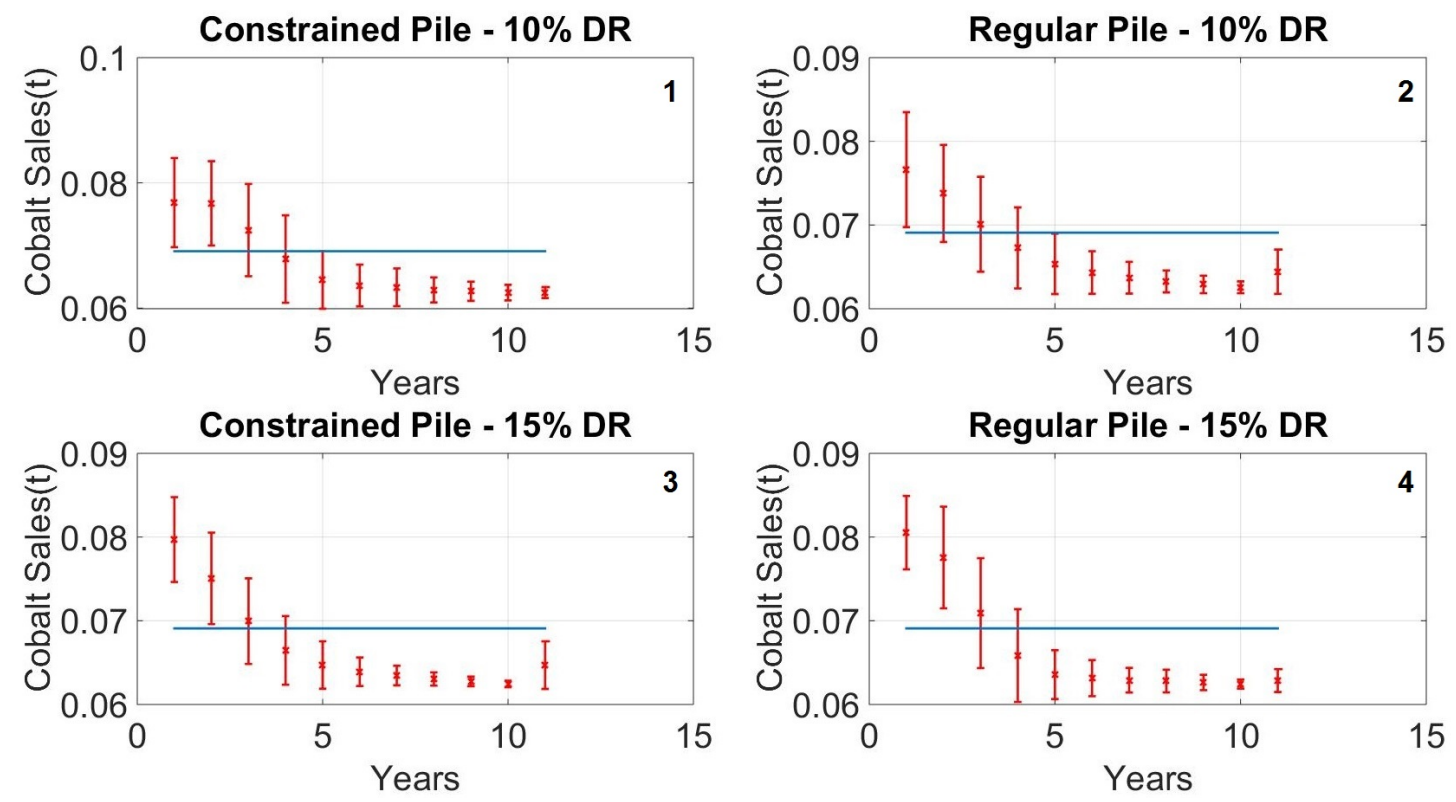

Figure 6.5: Cobalt sales plotting - Base study
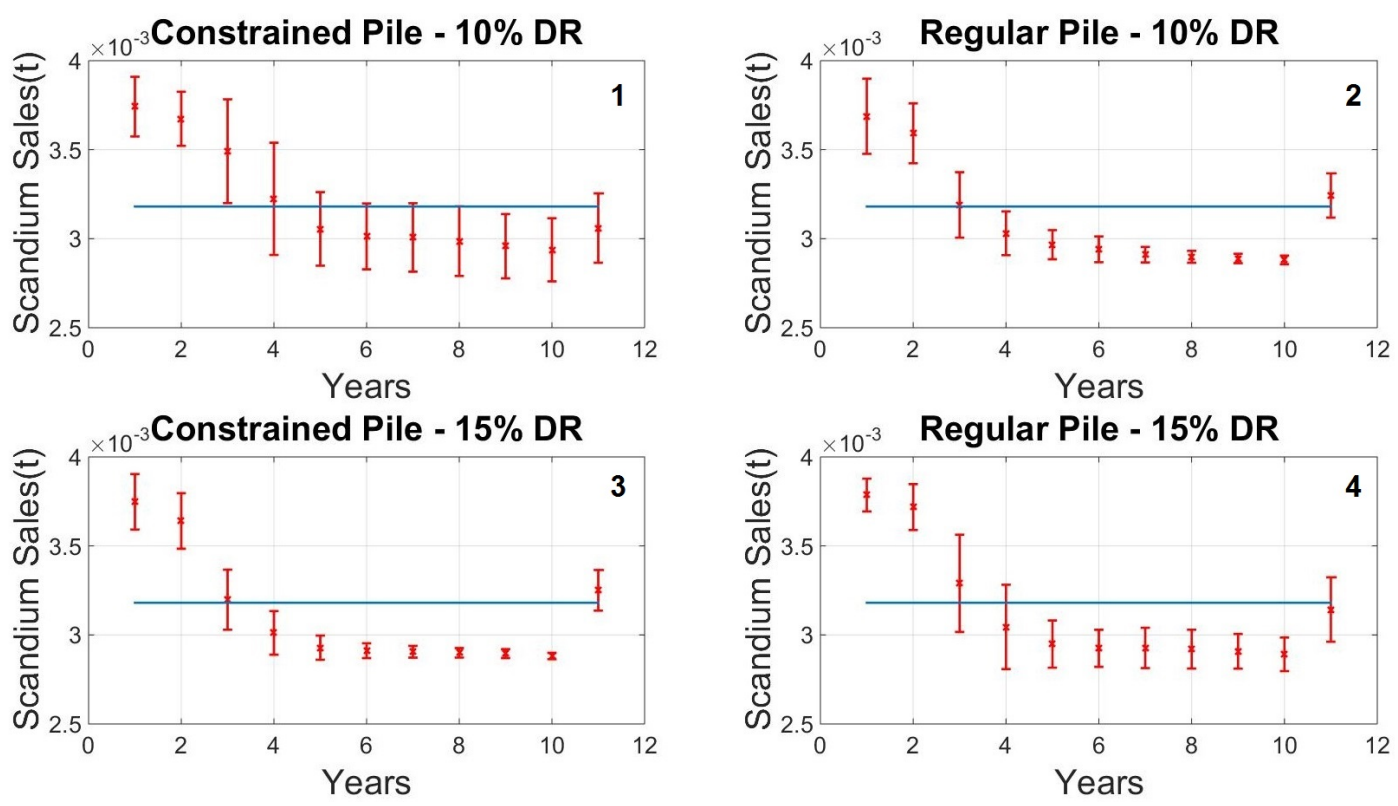

Figure 6.6: Scandium sales plotting - Base study 


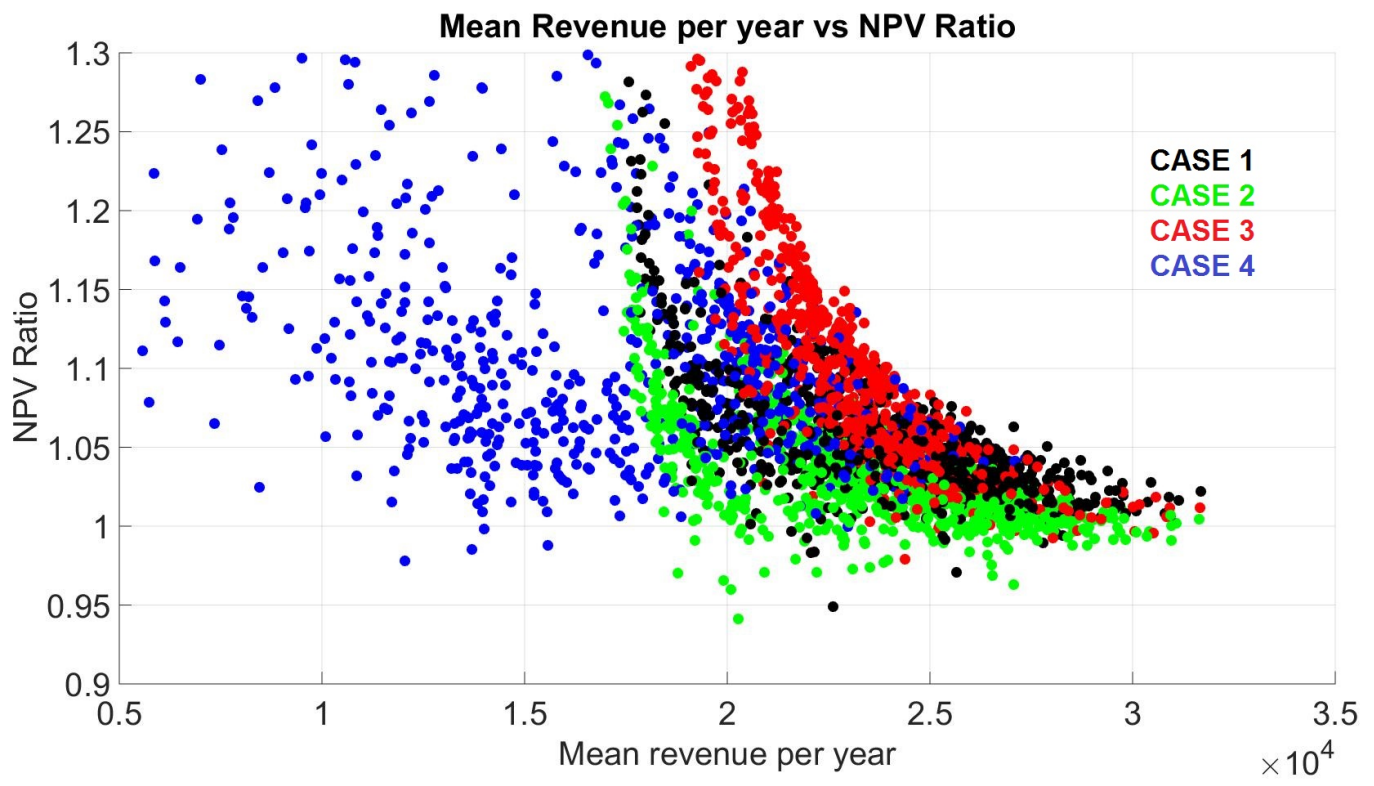

Figure 6.7: Revenue x Net Present Value Ratio - Base study

\subsection{Case Study - Greenvale Deposit}

The case study follows the same principles of the base study presented previously, with the following modifications on input parameters:

- Total reserve: $3.5 \mathrm{Mt}$ of ore at $0.98 \% \mathrm{Ni}, 0.08 \% \mathrm{Co}$ and $39 \mathrm{~g} / \mathrm{t} \mathrm{Sc}$

- Mine yearly throughput: 350,000 tonnes/annum

- Mill yearly throughput: 320,000 tonnes/annum

- Throughput modification allowed: $+-5 \%$ of the default capacity

- Buffer stockpile maximum capacity: 30,000 tonnes (constrained) and 70,000 tonnes (regular)

- Sales rate: At least 95\% of the produced capacity

Table 6.2 presents the results for the case study. 
Table 6.2: Case study - Result data

\begin{tabular}{|c|c|c|c|c|c|c|c|c|}
\hline \multirow{2}{*}{ Scenario } & \multicolumn{2}{|c|}{ CASE 1 } & \multicolumn{2}{c|}{ CASE 2 } & \multicolumn{2}{c|}{ CASE 3 } & \multicolumn{2}{c|}{ CASE 4 } \\
\cline { 2 - 9 } & $\begin{array}{c}\text { NPV } \\
\text { (US\$ } \\
\text { Mi) }\end{array}$ & $\begin{array}{c}\text { Increase } \\
\text { over } \\
\text { DCF } \\
(\%)\end{array}$ & $\begin{array}{c}\text { NPV } \\
\text { (US\$ } \\
\text { Mi) }\end{array}$ & $\begin{array}{c}\text { Increase } \\
\text { over } \\
\text { DCF } \\
(\%)\end{array}$ & $\begin{array}{c}\text { NPV } \\
\text { (US\$ } \\
\text { Mi) }\end{array}$ & $\begin{array}{c}\text { Increase } \\
\text { over } \\
\text { DCF } \\
(\%)\end{array}$ & $\begin{array}{c}\text { NPV } \\
\text { (US\$ } \\
\text { Mi) }\end{array}$ & $\begin{array}{c}\text { Increase } \\
\text { over } \\
\text { DCF } \\
(\%)\end{array}$ \\
\hline 1 & 142.15 & $4.58 \%$ & 140.51 & $2.01 \%$ & 86.51 & $12.71 \%$ & 84.74 & $7.39 \%$ \\
\hline 2 & 165.64 & $3.24 \%$ & 164.14 & $1.16 \%$ & 104.46 & $9.38 \%$ & 102.78 & $5.28 \%$ \\
\hline 3 & 198.21 & $1.64 \%$ & 197.05 & $0.14 \%$ & 128.53 & $6.36 \%$ & 127.09 & $3.41 \%$ \\
\hline 4 & 216.12 & $1.02 \%$ & 215.36 & $-0.17 \%$ & 142.23 & $5.21 \%$ & 140.74 & $2.52 \%$ \\
\hline 5 & 141.69 & $4.75 \%$ & 140.08 & $2.05 \%$ & 85.63 & $11.80 \%$ & 83.90 & $7.67 \%$ \\
\hline 6 & 153.82 & $3.92 \%$ & 152.28 & $1.59 \%$ & 95.28 & $11.16 \%$ & 93.41 & $6.24 \%$ \\
\hline 7 & 193.82 & $1.81 \%$ & 192.53 & $0.15 \%$ & 125.55 & $6.75 \%$ & 123.84 & $3.39 \%$ \\
\hline 8 & 212.69 & $1.45 \%$ & 211.48 & $0.02 \%$ & 139.99 & $5.72 \%$ & 138.33 & $2.85 \%$ \\
\hline 9 & 57.69 & $11.09 \%$ & 57.34 & $7.93 \%$ & 12.05 & $18.50 \%$ & 11.54 & $20.45 \%$ \\
\hline 10 & 83.29 & $7.01 \%$ & 82.98 & $3.96 \%$ & 32.76 & $18.23 \%$ & 32.05 & $16.80 \%$ \\
\hline 11 & 170.36 & $1.07 \%$ & 169.97 & $-0.27 \%$ & 102.11 & $7.31 \%$ & 101.35 & $4.09 \%$ \\
\hline 12 & 197.25 & $0.62 \%$ & 196.74 & $-0.55 \%$ & 123.29 & $5.58 \%$ & 122.38 & $2.84 \%$ \\
\hline 13 & 64.82 & $11.04 \%$ & 64.61 & $6.87 \%$ & 17.33 & $16.21 \%$ & 16.86 & $12.75 \%$ \\
\hline 14 & 80.21 & $7.69 \%$ & 79.98 & $4.45 \%$ & 30.31 & $19.50 \%$ & 29.61 & $15.09 \%$ \\
\hline 15 & 173.42 & $1.52 \%$ & 172.84 & $0.05 \%$ & 104.30 & $7.58 \%$ & 103.43 & $4.59 \%$ \\
\hline 16 & 194.49 & $1.05 \%$ & 193.72 & $-0.31 \%$ & 121.72 & $6.07 \%$ & 120.77 & $3.23 \%$ \\
\hline Average & - & $3.97 \%$ & - & $1.82 \%$ & - & $10.51 \%$ & - & $7.41 \%$ \\
\hline
\end{tabular}

The analysis of the numerical results presented in this study reinforces the positive outcomes of stockpiling material up to a certain maximum capacity. In all of the cases, with the exception of scenario 9 on Case 4, the method of constrained stockpiling provided a better NPV mean for the same forecasted commodity prices.

In fact, some of the scenarios presented in Case 2 were not able to achieve the NPV of the standard method. They correspond to scenarios 4, 11, 12 and 16. All of them are based on higher trends for commodity prices, and distance their results less than $1 \%$ from the DCF methodology.

The same scenarios were rerun with higher number of function evaluations and decreased the gap between standard and dynamic values. In fact, Scenario 4 was able to be more profitable, which is an indication that the number of function evaluations 
is a crucial parameter for evaluation. Nevertheless, the cases presented the following mean values of NPV increase: $3.97 \%, 1.82 \%, 10.51 \%$, and $7.41 \%$, for cases 1 to 4 , respectively.

Figures 6.8 to 6.13 show the plots of operating parameters under study. Regarding mine throughput, the optimized values were all observed being under the default capacity of $350 \mathrm{k}$ tonnes. They were more scattered for Case 1, while Case 2 observed almost no modification or deviation ffrom the minimum allowed values.
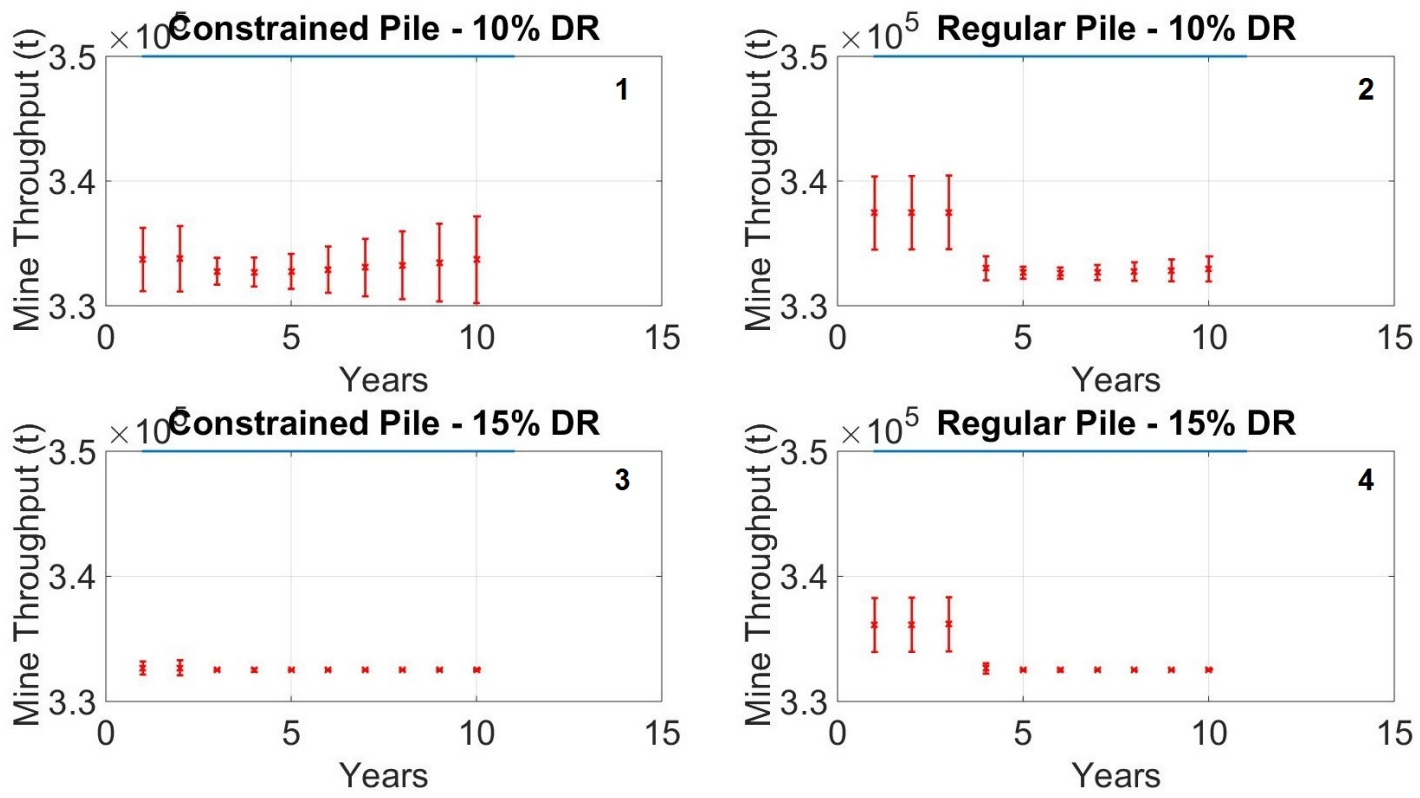

Figure 6.8: Mine throughput plotting - Case study

The mill was also operated in lower capacity for all the cases investigated. Cases 1 and 3 presented a small increase in the first year and a subsequent drop on the later years. On the other hand, cases 2 and 4 have an increasing mill capacity (contained within the lower boundary of rated capacity) for the first 2 years, and a subsequent decrease posteriorly.

Buffer pile configuration was not significantly different among cases. The ones with higher inventory capacity achieve limit by the third year, while the other cases reach full capacity by the end of the second year. There is not much variability after the full capacity is reached. 

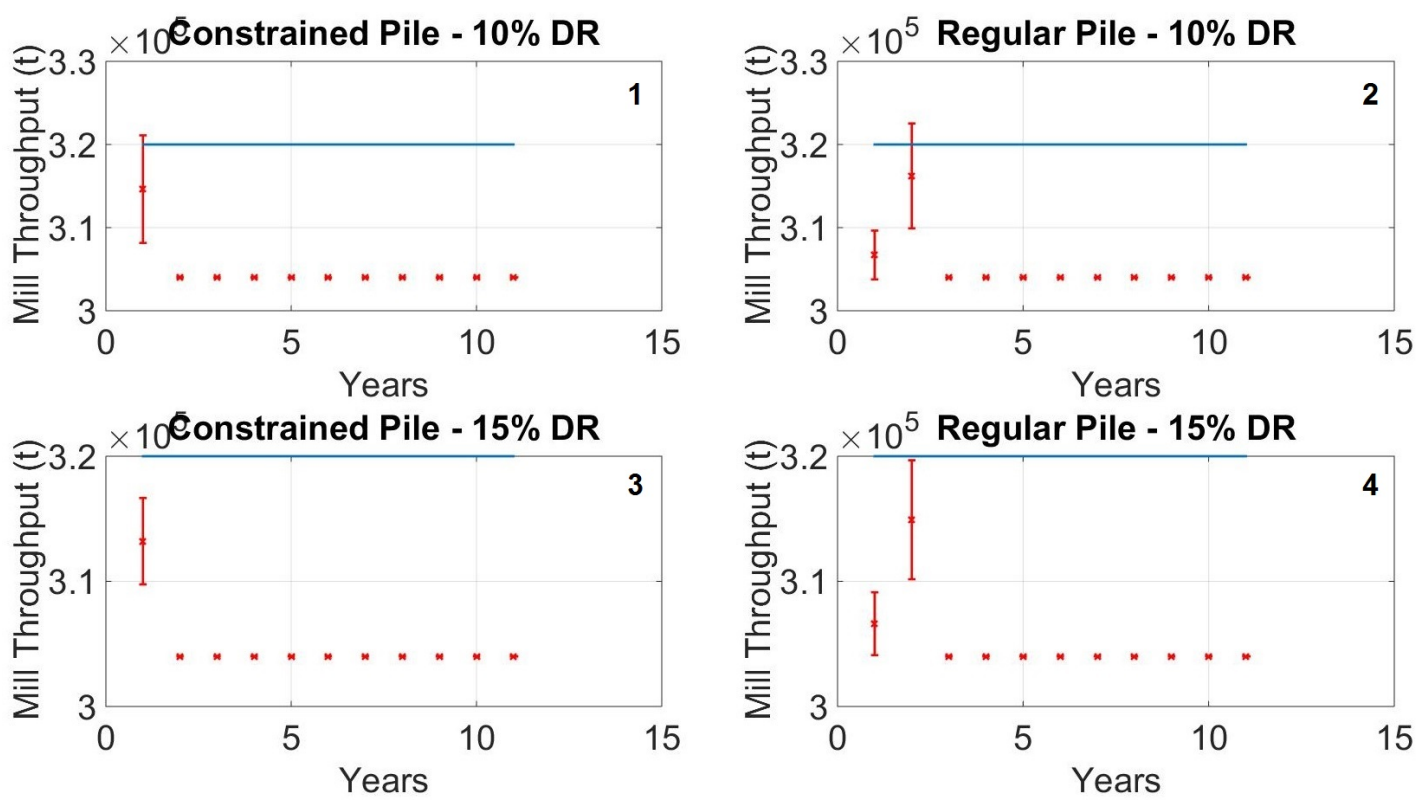

Figure 6.9: Mill throughput plotting - Case study
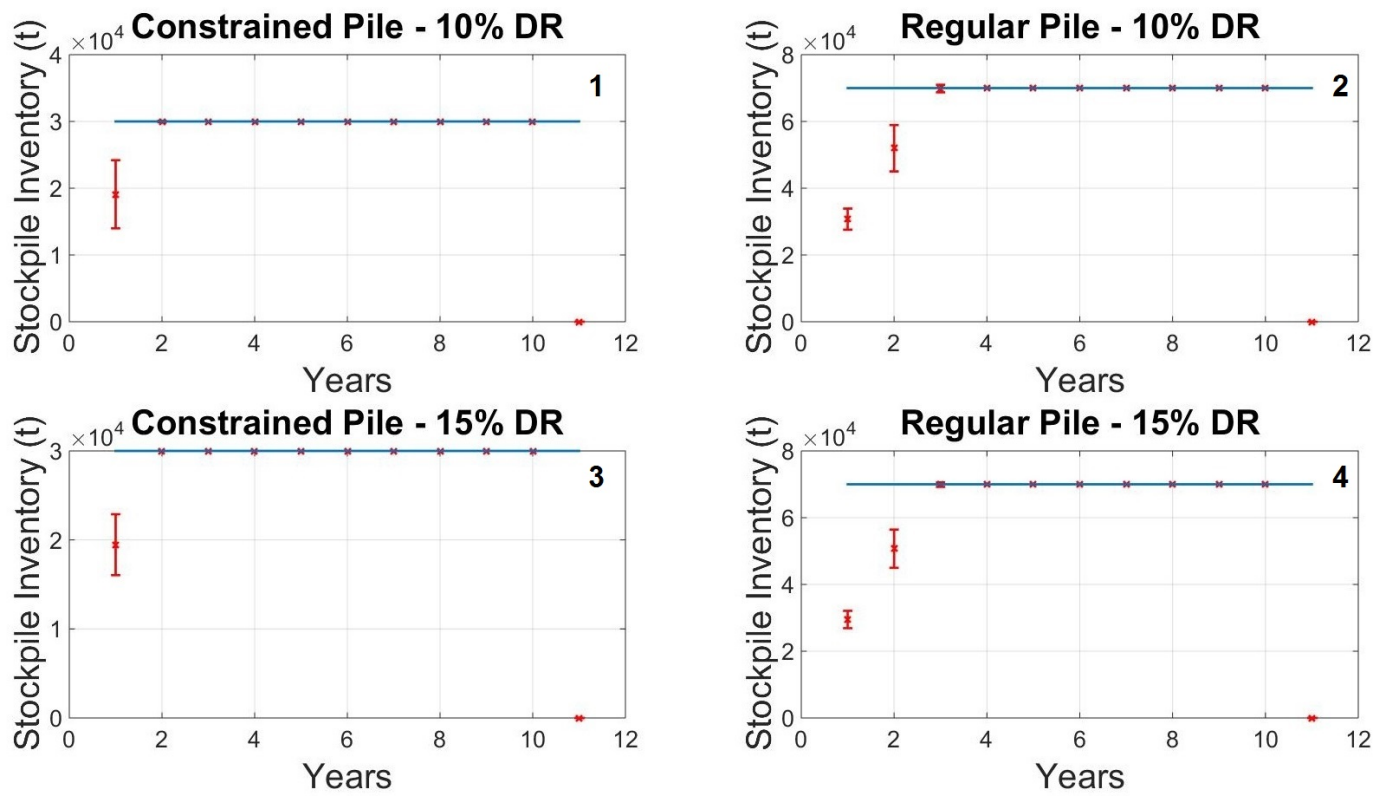

Figure 6.10: Buffer pile inventory plotting - Case study 
Commodity sales present a characteristic behavior for the three commodities. Given the increase in mill throughput on years 1 and 2, the commodities are then stored on the product pile (not available for visualization on the plots) and subsequently sold at maximum capacity from years 3 to 5 , obtaining maximum revenue for product. Scandium sales are slightly different because they occur with more intensity in the early years, for cases with constrained stockpile.
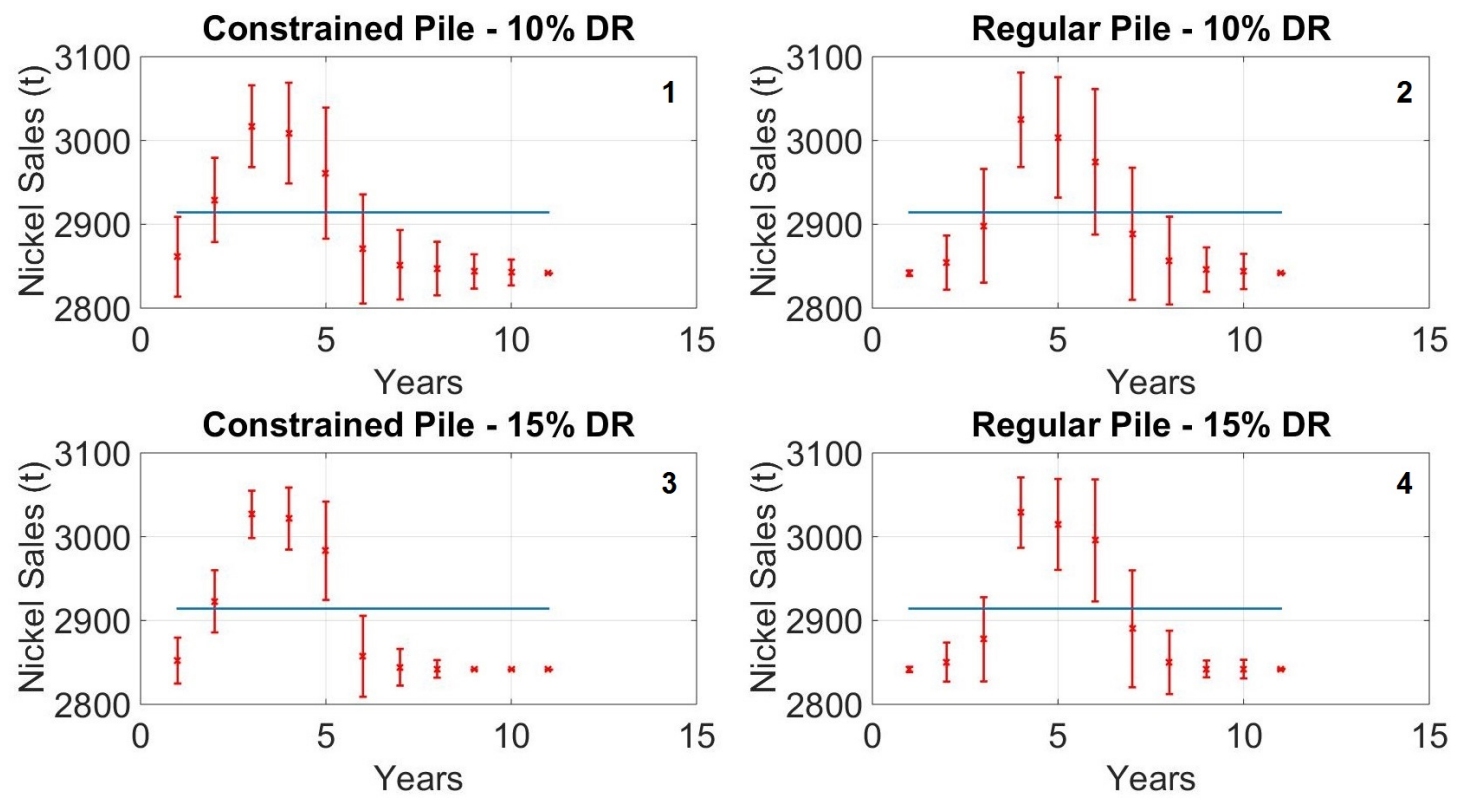

Figure 6.11: Nickel sales plotting - Case study

Finally, the scatterplot of mean revenues compared to NPV ratio is presented in Figure 6.14. It shows the particular behavior of mean yearly revenues for iterations, in which lower values for a particular simulation tend to obtain higher NPV increase compared to the DCF method.

Also, the graph presents higher decrease in profit with the use of constrained buffer stockpiles. For equivalent revenue values, cases 1 and 3 present higher percentage increase. Finally, the cases with highest NPV ratio are the ones with higher discount rate. 

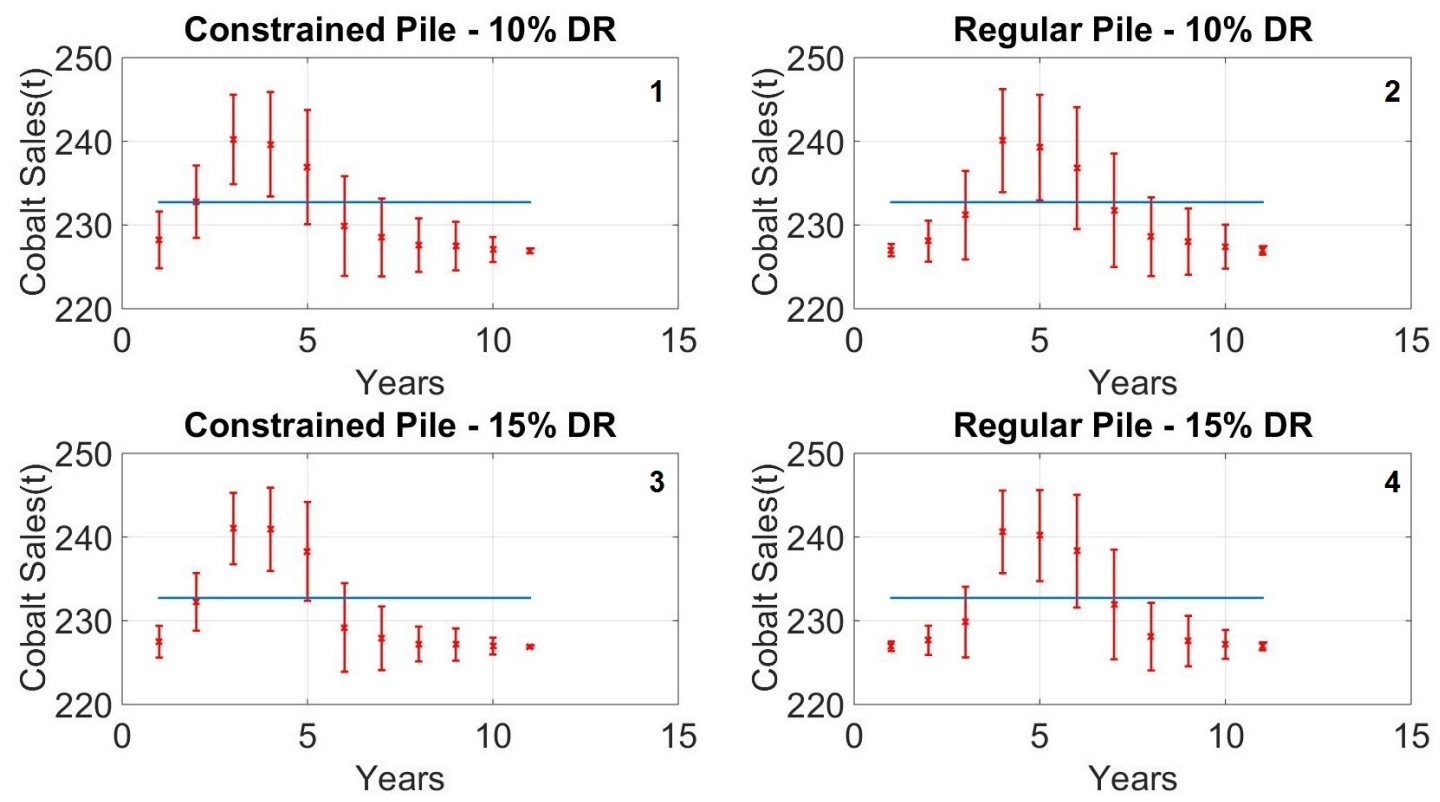

Figure 6.12: Cobalt sales plotting - Case study
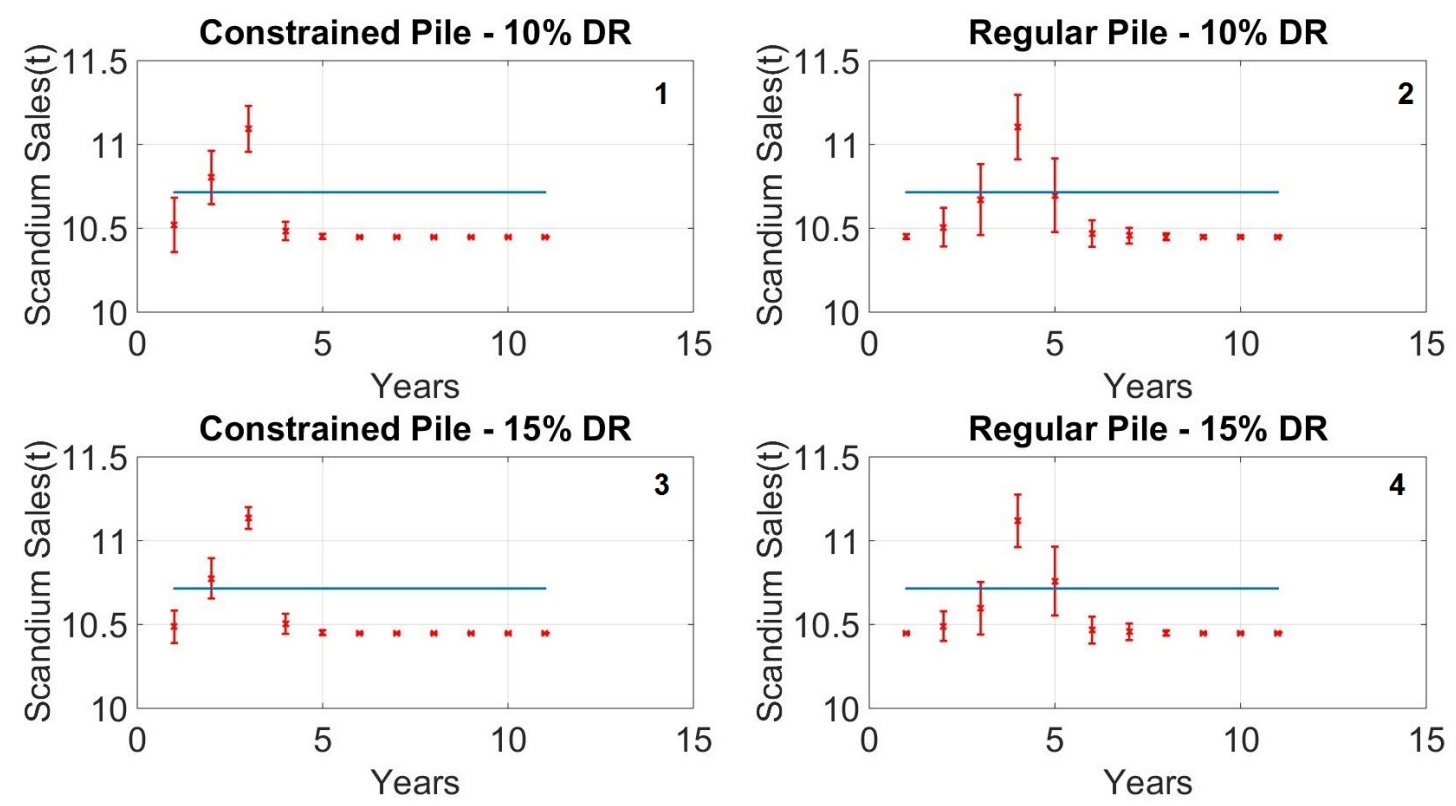

Figure 6.13: Scandium sales plotting - Case study 


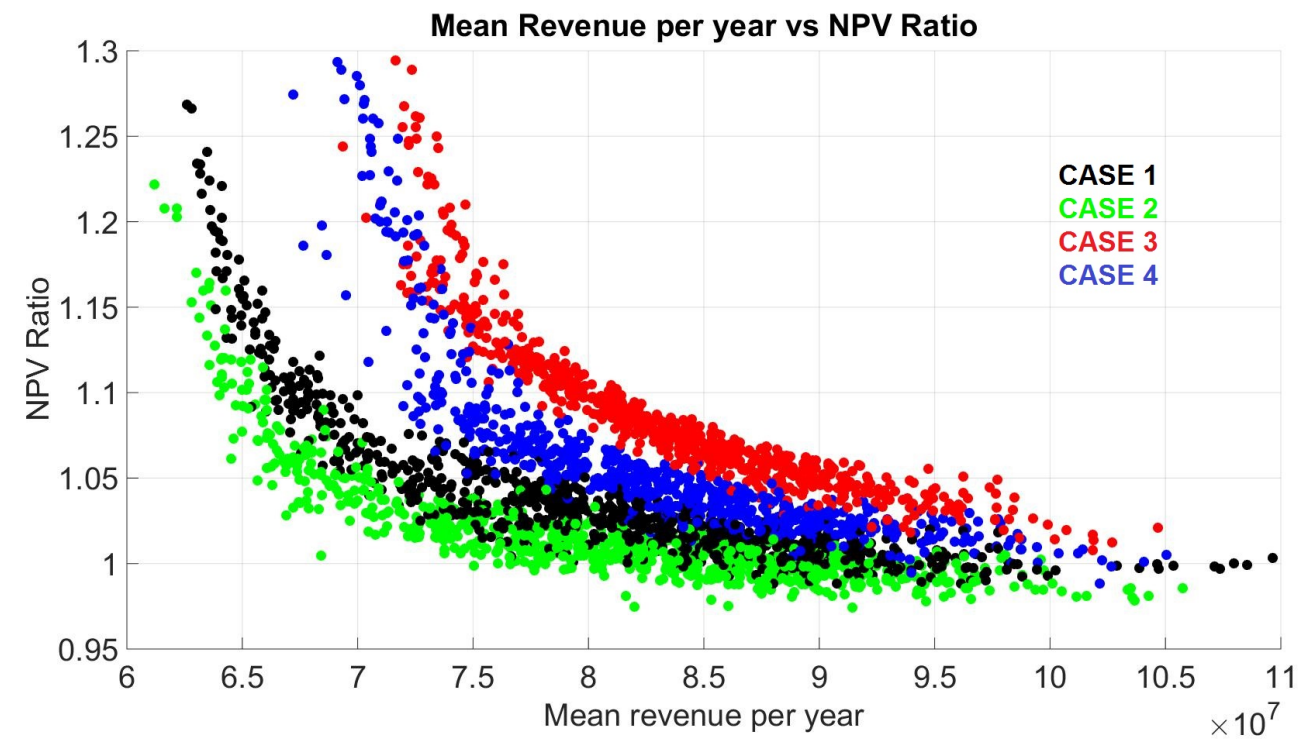

Figure 6.14: Revenue x Net Present Value Ratio - Case study

\subsection{Discussion}

The numerical results corroborate the positive impact of dynamic modeling for mineral valuation. However, they were also able to showcase the two main shortcomings of the DOV method for the industrial data:

- The need to run as many function evaluation as possible while minimizing time

- Difficulty to obtain optimal parameters with growing commodity prices

A few selected number of price scenarios with growing commodity value trend failed to achieve higher NPV values with the default number of function evaluations provided. Therefore, one of the conclusions was that the interior point method, even though reported as a fast and efficient methodology to identify optimal values, required more function evaluations to perform in an optimal way. However, the vast majority of these values were obtained from scenarios with geometric brownian motion price forecasts used as input. It was observed previously that GBM cannot be fully modeled to simulate a specific market scenario. Its stochastic configuration 
distorts values, specially after more than 5 years of simulated paths. Therefore, the results generated from such methodology are valid, but the ones generated through mean-reverting drift model input are preferrable.

The dynamic model, when subjected to an economic scenario of highly increasing prices, tends to determine a net present value virtually identical to the standard discounted cash method. The buffer pile necessity is practially non-existent in this context.

The reason for such disparity is because the optimization's starting point is on the lower boundary of the input parameter vector. Without the appropriate number of function evaluations to test all possible paramater values, and also relying on a local minimum methodology, the dynamic model may fail to identify the optimal values under specific market scenarios. The issue, conclusively, relies on a combination of rising prices and lack of proper functions evaluations.

\subsubsection{Analysis of Variance}

The results were finally subjected to analysis of variance, for identification of the most significant parameters responsible for variability of NPV. More specifically, the NPV ratio values were analyzed on a N-way ANOVA methodology, with 6 factors and 2 levels each. The parameters are described below:

- Forecasting type (FType): HWV and GBM

- Price Volatility (Sigma): Regular and High

- Nickel trend (Ni) and Cobalt trend (Co): Low and High

- Buffer stockpile size (Pile): COnstrained and Regular

- Discount Rate (DRate): $10 \%$ and 15\%

Table 6.3 shows the ANOVA results for the Base study. Notably, the most significant parameter responsible for variability is discount rate, closely followed by nickel

price trend. This is due to the higher mean squared error (MSE) in these two parameters. The other parameters are not significally impactant, and corresponding to 
the following, in decreasing order of impact for variability: Cobalt price trend, buffer stockpile size, forecasting type and prive volatility.

\begin{tabular}{llllll}
\multicolumn{5}{c}{ Table } & 6.3 : Analysis of Variance table - Base study \\
\hline Source & Sum Sq. & d.f. & Mean Sq. & F ratio & Prob \\
\hline FType & 0.054 & 1 & 0.05398 & 14.44 & 0.0001 \\
Sigma & 0.0026 & 1 & 0.0026 & 0.69 & 0.4047 \\
Ni & 4.5715 & 1 & 4.57147 & 1223.12 & 0 \\
Co & 0.126 & 1 & 0.12596 & 33.7 & 0 \\
Pile & 0.1237 & 1 & 0.12368 & 33.09 & 0 \\
DRate & 4.5868 & 1 & 4.58684 & 1227.24 & 0 \\
Error & 11.3247 & 3030 & 0.00374 & & \\
Total & 20.1504 & 3036 & & & \\
\hline
\end{tabular}

Table 6.4 corresponds to the ANOVA results for the case study. The parameters under analysis are presented in order of significance for overall variability: Nickel price trend, discount rate, forecasting type, buffer stockpile size, cobalt price trend and price volatility.

\begin{tabular}{llllll}
\multicolumn{5}{c}{ Table } & 6.4: \\
Source & Sum Sq. & d.f. & Mean Sq. & F ratio & Prob \\
\hline FType & 0.9235 & 1 & 0.92348 & 248.22 & 0 \\
Sigma & 0.0091 & 1 & 0.0091 & 2.45 & 0.1179 \\
Ni & 3.3644 & 1 & 3.36436 & 904.3 & 0 \\
Co & 0.0331 & 1 & 0.03307 & 8.89 & 0.0029 \\
Pile & 0.5572 & 1 & 0.55724 & 149.78 & 0 \\
DRate & 2.8409 & 1 & 2.84085 & 763.59 & 0 \\
Error & 11.124 & 2990 & 0.00372 & & \\
Total & 17.9017 & 2996 & & & \\
\hline
\end{tabular}

Nickel price had the greatest impact, followed closely by the discount rate. Given a context of steady feed grades for the processing plant, industrial operating scale and vast amounts of material produced, the most relevant factor for revenue generation, and subsequently profit, was commodity price. Since grade was not evaluated specifically in this study, commodity price was the main parameter for differences in NPV. 


\section{Chapter 7}

\section{Conclusion and Recommendations}

The complex system proposed in this research was able to perform mineral asset valuation for diversified economic scenarios and multiple mineral commodities. It relies on a novel model for determining the operating parameters for mine, mill/smelter and commodity sales. The methodology, denominated dynamic option valuation (DOV), is a stochastic method based on operational flexibility and buffer stages between the main operations.

Two main studies were proposed and presented in this project. Both of them are based on a hypothetical Ni-Co-Sc deposit subjected to different levels of commodity trend, volatility, discount rate and maximum buffer stockpile capacity. Overall, the dynamic option valuation was able to obtain NPV results ranging from an average of $2 \%$ to $11 \%$ higher than a standard discounted cash flow methodology. Also, the DOV model was able to achieve a $2 \%$ NPV increase in comparison to an example conducted using the Real Option Valuation (ROV) methodology.

Fundamentally, the DOV represents real dynamics that mine mine operators and managers will employ as prices trend up or down. Different price scenarios played a significant role for the identification of optimal operating parameters for the proposed operation. In a context of mining without variability in the feed grade, commodity price is the ultimate factor for variability in NPV determination of industrial-scale mining projects, followed closely by the discount rate in use.

The other significant parameter for determining optimal operating parameters corresponds to stockpile buffer size. For the vast majority of economic scenarios investigated, the ones with constrained buffer piles performed better during mineral asset valuation, compared to the equivalent cases with regular buffer pile inventory. Maintaining a lower maximum inventory size allows the optimizer to find the optimal NPV 
value using a lower number of function evaluations. Also, adding more constraints to the mine valuation model allows faster result generation.

Price volatility was the least significant parameter of impact for the valuation process, considering that the volatility was hedged by multiple simulations. On standalone cases, however, it possesses greater impact. Scenarios with higher volatility presented higher percentage increase over the DCF method.

Regarding the two methodologies used to generate price forecasts, commodity prices generated through the mean-reverting drift model worked more successfully with the valuation model, given its higher capacity of manipulation by the user. Finally, the proposed optimization methods were successful in generating optimal NPV values for the hypothetical mining project specified in this report.

Recommendations for future include the use of varying feed grade for mine planning and observing how grade uncertainty can be associated with commodity price uncertainty on mineral asset valuation. One of crucial assumptions in this research was the use of non-selective mining methods, targeting a stable feed grade for operation. The assumption is valid, although a more realistic procedure, specially for open-pit metal mines, would be the use of selective mining. This methodology will require an even more robust type of optimization to account for uncertainty of both parameters.

Other commodities (such as coal, iron and gold) may be evaluated by the DOV. These also have great importance in the global economy, and have been greatly affect by price volatility in the recent years. Recent studies attempting to recover rare metals and REEs from these major commodities can benefit from financial evaluation performed by a dynamic methodology.

In addition, commodity supply and demand be studied as a new parameter of analysis. Product sales on the valuation model can be constrained by the available supply or demand in the market, generating new possibilities of mineral asset valuation under sales restrictions.

Finally, the study of material stockpiling can be expanded for identification of an optimal maximum buffer stockpile size for mining projects. The proposed scenarios with constrained buffer piles obtained the highest increase in NPV, compared to the standard methodology. Accurate mineral inventory management would benefit mining companies, buyers and investors. 


\section{Bibliography}

[Anderson, 2004] Anderson, P. (2004). Business Economics and Finance with MAT$L A B, G I S$, and Simulation Models. Chapman and Hall/CRC, first edition.

[Asad, 2005] Asad, M. W. A. (2005). Cutoff grade optimization algorithm with stockpiling option for open pit mining operations of two economic minerals. International Journal Of Surface Mining Reclamation And Environment Reclamation And Environment, 3:176-187.

[Bowman and Husain, 2004] Bowman, C. and Husain, A. (2004). Forecasting commodity prices: Futures versus judgment.

[Brandimarte, 2006] Brandimarte, P. (2006). Numerical Methods in Finance and Economics: A MATLAB-Based Introduction. Wiley-Interscience, second edition.

[Brown et al., 2008] Brown, O., Crawford, A., and Gibson, J. (2008). From feast to famine: After seven (relatively) good years, what now for commodity producers in the developing world? International Institute for Sustainable Development.

[Caldentey et al., 2004] Caldentey, R., Epstein, R., and Saure, D. (2004). A real options approach for optimizing long-term mining plans. Industry Engineering Department, University of Chile.

[Chen, 2002] Chen, S.-H. (2002). Genetic Algorithms and Genetic Programming in Computational Finance. Springer.

[Commission, 2008] Commission, C. F. T. (2008). Staff report on commodity swap dealers \& index traders with commission recommendations. $<$ http://www.cftc.gov/idc/cftcstaffreportonswapdealers09.pdf $>$.

[Connerly, 2014] Connerly, W. (2014). Commodity price volatility: What should users do? Forbes Magazine. 
[Croew et al., 2006] Croew, A., McClean, C., and Cresser, M. (2006). An application of genetic algorithms to the robust estimation of soil organic and mineral fraction densities. Environmental Modelling \& Software, 21(10):1503-1507.

[Crundwell, 2011] Crundwell, F. (2011). Extractive Metallurgy of Nickel, Cobalt and Platinum Group Metals. Elsevier, first edition.

[Cuddington and Zellou, 2013] Cuddington, J. and Zellou, A. (2013). A simple mineral market model: Can it produce super cycles in prices? Resources Policy, $38: 75-87$.

[Dagdelen, 1992] Dagdelen, K. (1992). In Cutoff grade optimization, pages 157165. 23rd International Symposium on Application of Computers and Operations Research in Minerals Industries.

[Darling, 2013] Darling, P. (2013). SME Mining Engineering Handbook. Society for Mining, Metallurgy, and Exploration (SME), third edition.

[Davis, 2010] Davis, G. (2010). Trade in mineral resources. World Trade Report 2010.

[Deng, 1999] Deng, S. (1999). Stochastic models of energy commodity prices and their applications: Mean-reversion with jumps and spikes. Manual.

[Driels and Shin, 2004] Driels, M. and Shin, Y. (2004). Determining the number of iterations for monte carlo simulations of weapon effectiveness. Defense Threat Reduction Agency.

[Fischer Black, 1973] Fischer Black, M. S. (1973). The pricing of options and corporate liabilities. The Journal of Political Economy, 81:637-654.

[Forsgren et al., 2002] Forsgren, A., Gill, P., and Wright, M. (2002). Interior methods for nonlinear optimization. SIAM Review, 44:525-597.

[Geman, ] Geman, H. Mean reversion versus random walk in oil and natural gas prices. Advances in Mathematical Finance, pages 219-228.

[Gilbertie, 2012] Gilbertie, S. (2012). Commodities in a portfolio - what do they bring to the table? Journal of Indexes, pages 18-25.

[Glasserman, 2004] Glasserman, P. (2004). Monte Carlo Methods in Financial Engineering. Springer, New York. 
[Goldberg, 1989] Goldberg, D. (1989). Genetic Algorithms in Search, Optimization, and Machine Learning. Addison-Wesley Professional, first edition.

[Gupta et al., 2005] Gupta, V., Mohanty, M., Mahajan, A., and Biswal, S. (2005). Genetic algorithms - a novel technique for optimization of coal preparation plant. In International Seminar on Mineral Processing Technology - 2005 Proceedings.

[Hamilton, 2009] Hamilton, J. (2009). Causes and consequences of the oil shock of 2007-2008. UC San Diego.

[Haque et al., 2014] Haque, A., Topal, E., and Lilford, E. (2014). A numerical study for a mining project using real options valuation under commodity price uncertainty. Resources Policy, 39:115-123.

[Hassan, 2005] Hassan, R. (2005). Value-at-risk analysis for real options in complex engineered systems. MIT. MSc Thesis.

[Holland, 1975] Holland, J. (1975). Adaptation in Natural and Artificial Systems. University of Michigan Press, University of Michigan, first edition.

[Houck et al., 1995] Houck, C., Joines, J., and Kay, M. (1995). A genetic algorithm for function optimization: A matlab implementation. Independent Research.

[Hummels and Skiba, 2004] Hummels, D. and Skiba, A. (2004). Shipping the good apples out: An empirical confirmation of the alchian-allen conjecture. Journal of Political Economy, 112:1384-1402.

[Humphries, 2013] Humphries, M. (2013). Rare earth elements: The global supply chain. Congressional Research Service.

[InvestentMine, 2016] InvestentMine (2016). Commodity and metal prices.

[Jacks et al., 2009] Jacks, D., O’Rourke, K., and Williamson, J. (2009). Commodity price volatility and world market integration since 1700. Institute for International Integration Studies.

[Kaplan, 2012] Kaplan, P. (2012). Beyond beta-passive alternatives to active commodities strategies. $<$ https://corporate.morningstar.com/CommodityIndexWhitePaper.pdf $>$.

[Kienitz and Wetterau, 2013] Kienitz, J. and Wetterau, D. (2013). Financial Modelling: Theory, Implementation and Practice with MATLAB Source. Wiley. 
[Kilian, 2009] Kilian, L. (2009). Not all oil price shocks are alike: Disentangling demand and supply shocks in the crude oil market. American Economic Review, 99:1053-1069.

[Krugman, 2008] Krugman, P. (2008). Fuels on the hill.

[Law and McComas, 2000] Law, A. and McComas, M. (2000). Simulation-based optimization. In Simulation Conference, 2000.

[Masters, 2008] Masters, M. (2008). Testimony before the committee on homeland security and governmental affairs. US Senate.

[Mathworks, 2015] Mathworks (2015). Monte carlo simulation. http://www.mathworks.com/discovery/monte-carlo-simulation.html.

[Mathworks, 2016a] Mathworks (2016a). Documentation for Optimization Toolbox. Mathworks.

[Mathworks, 2016b] Mathworks (2016b). Matlab parametric models. $\quad<$ http://www.mathworks.com/help/finance/parametricmodels.html?refresh=truebriahp4-1>.

[McDaniel and Driebe, 2005] McDaniel, R. and Driebe, D. (2005). Uncertainty and Surprise in Complex Systems - Uncertainty and Surprise in Complex Systems. Springer.

[Mills, 2012] Mills, R. (2012). Nickel mining like its 1864. $<$ http://aheadoftheherd.com/Newsletter/2012/Nickel-Mining-Like-its1864.htm>.

[MLM, 2013] MLM (2013). Sconi project - environmental impact assessment. Queensland Government - Department of Environment and Heritage Protection.

[MLM, 2015] MLM (2015). Metallica minerals - quarterly report to 31 dec 2014. $<$ http://www.metallicaminerals.com.au/quarterly-report $>$.

[Mohammad, 1997] Mohammad, W. (1997). Multi-mineral cutoff grade optimization with option to stockpile. Master's thesis, Colorado School of Mines.

[Mohammad and Khan, 2004] Mohammad, W. and Khan, M. (2004). Management of open pit mining operations with ore stockpiles. Journal of Engineering and Applied Sciences, 36(12). 
[Montiel and Dimitrakopoulos, 2015] Montiel, L. and Dimitrakopoulos, R. (2015). Optimizing mining complexes with multiple processing and transportation alternatives: An uncertainty-based approach. European Journal of Operational Research, 247(1):166-178.

[Mueller, 2004] Mueller, G. (2004). Local vs. global optimization in syntax: A case study. In Workshop on Variation within Optimality Theory, Stockholm University: Institute of Linguistics.

[Nadig, 2014] Nadig, D. (2014). Smart beta grows in commodity etfs. Exchange Traded Funds.

[Nassar et al., 2015] Nassar, N., Graedel, T., and Harper, E. (2015). By-product metals are technologically essential but have problematic supply. Science Advances, $1(3)$.

[Nieto and Zhang, 2013] Nieto, A. and Zhang, K. (2013). Cutoff grade economic strategy for byproduct mineral commodity operation: rare earth case study. Transactions Of The Institution Of Mining And Metallurgy, Section A: Mining Technology, 122(3):166-171.

[Noble, 2014] Noble, A. (2014). Commodity beta index summary. unpublished.

[Noble and Luttrell, 2015] Noble, A. and Luttrell, G. (2015). Micro-pricing optimization: Value-based partition curve analysis with applications to coal separation. 2015 SME Annual Meeting Exhibit.

[Oksendal, 2003] Oksendal, B. (2003). Stochastic Differential Equations: An Introduction with Applications. Springer, Berlin, Germany.

[Pindyck, 1999] Pindyck, R. (1999). The long run evolution of energy prices. Energy Journal, 20(1):1-27.

[Pindyck, 2001] Pindyck, R. (2001). The dynamics of commodity spot and future markets: a primer. The Energy Journal, 22(3):1-29.

[Pindyck, 2004] Pindyck, R. (2004). Volatility and commodity price dynamics. The Journal of Futures Markets, 24(11):1029-1047.

[Postali and Picchetti, 2006] Postali, F. and Picchetti, P. (2006). Geometric brownian motion and structural breaks in oil prices: A quantitative analysis. Energy Economics, 28(4):506-522. 
[Powell, 1983] Powell, J. (1983). The asymptotic normality of two-stage least absolute deviations estimators. Econometria, 51(5):1569-1575.

[Radetzki, 2008] Radetzki, M. (2008). A Handbook of Primary Commodities in the Global Economy. Cambridge University Press, Cambridge.

[Rahman et al., 2013] Rahman, A., Dutta, R., and Smith, D. (2013). Exploring mineral domains with genetic algorithm. In The 2013 International Joint Conference on Neural Networks. IEEE.

[Ramazan and Dimitrakopoulos, 2013] Ramazan, S. and Dimitrakopoulos, R. (2013). Production scheduling with uncertain supply: a new solution to the open pit mining problem. Optimization and Engineering, 14(2):361-380.

[Rendu, 2014] Rendu, J.-M. (2014). An Introduction to Cut-Off Grade Estimation. Society for Mining, Metallurgy, and Exploration (SME), second edition.

[Richards et al., 1998] Richards, E., McKay, B., and Richards, W. (1998). Collective choice and mutual knowledge structures. Advances in Complex Systems, 1:221236 .

[Ritter et al., 2011] Ritter, F., Schoelles, M., Quigley, K., and Klein, L. (2011). Determining the number of simulation runs: Treating simulations as theories by not sampling their behavior. Human-in-the-Loop Simulations.

[Runge, 1998] Runge, I. (1998). Mining Economics and Strategy. Society for Mining, Metallurgy, and Exploration (SME).

[Salim and Cai, 1997] Salim, V. and Cai, X. (1997). A genetic algorithm for railway scheduling with environmental considerations. Environmental Modelling $\&$ Software, 12(4):301-309.

[Shafiee et al., 2009] Shafiee, S., Topal, M., and Nehring, M. (2009). Adjusted real option valuation to maximise mining project value - a case study using century mine. Melbourne, Victoria, Australia. Project Evaluation Conference.

[Shreve, 2004] Shreve, S. (2004). Stochastic Calculus for Finance II: ContinuousTime Models. Springer-Verlag.

[Simon, 1995] Simon, H. (1995). Near decompositability and complexity: How a mind resides in a brain. Complex adaptative systems, pages 25-43. 
[Smith, 2008] Smith, L. (2008). Commodity funds 101. <http://www.investopedia.com/articles/mutualfund/09/commodity-mutualfunds.asp $>$.

[Stuermer, 2014] Stuermer, M. (2014). 150 years of boom and bust: What drives mineral commodity prices? Federal Reserve Bank of Dallas Research Department, 1414 .

[Tang and Xiong, 2010] Tang, K. and Xiong, W. (2010). The financialisation of commodities.

[Trench and Sykes, 2014] Trench, A. and Sykes, J. (2014). Perspectives on mineral commodity price cycles and their relevance to underground mining. Australian Institute of Mining \& Metallurgy 12th Underground Operators Conference Proceedings.

[USGS, 2015] USGS (2015). Metals and minerals: U. s. geological survey minerals yearbook. <http://minerals.usgs.gov/minerals/pubs/commodity/myb/>.

[Vardy, 2015] Vardy, N. (2015). Use smart beta to beat the index funds. $<$ http://www.marketwatch.com/story/use-smart-beta-to-beat-the-index-funds $>$.

[Vasant, 2012] Vasant, P. (2012). Meta-Heuristics Optimization Algorithms in Engineering, Business, Economics, and Finance. IGI Global, first edition.

[Veld, 2014] Veld, D. (2014). Complex systems in financial economics: Applications to interbank and stock markets. PhD thesis, Amsterdam School of Economics Research Institute.

[Wang et al., 2011] Wang, W., Pranolo, Y., and Cheng, C. (2011). Metallurgical processes for scandium recovery from various resources: A review. Hydrometallurgy, 108(1):100-108.

[Warnelid, 2011] Warnelid, R. (2011). Financial modelling of stockpiles in a mining project. <http://www.corality.com/training/campus/post/modelling-stockpilesin-a-mining-project $>$.

[WBG, 2014] WBG (2014). Global Economic Prospects. Commodity Markets Outlook - World Bank Group.

[Willingham, 2013] Willingham, D. (2013). Real options valuation with matlab: A mining economics case study. $<$ https://www.mathworks.com/matlabcentral/linkexchange/links/3712 $>$ F. 
[Willis and Gifford, 2010] Willis, B. and Gifford, M. (2010). Technical report on the agata nickel project scoping study. <http://www.mindoro.com/i/pdf/10-11-19agata-ni-scoping-study.pdf $>$.

[Yang, 2012] Yang, Z. (2012). Geometric brownian motion model in financial market. $<$ http: $/ /$ citeseerx.psu.edu/download?doi $=10.1 .1 .449 .7204$ rep $=$ rep1type $=$ pdf $>$.

[Yun et al., 1990] Yun, Q., Liu, J., Chen, Y., and Huang, G. (1990). Optimization of planning and design in underground mines. 22nd Symposium APCOM, pages $255-262$.

[Zhang et al., 2015] Zhang, K., Nieto, A., and Kleit, A. (2015). The real option value of mining operations using mean-reverting commodity prices. Mineral Economics, $28(1): 11-22$. 